\title{
Understanding HIV-related stigma : social and psychological processes
}

Citation for published version (APA):

Stutterheim, S. E. (2011). Understanding HIV-related stigma : social and psychological processes.

[Doctoral Thesis, Maastricht University]. Universiteit Maastricht. https://doi.org/10.26481/dis.20110211ss

Document status and date:

Published: 01/01/2011

DOI:

$10.26481 /$ dis.20110211ss

Document Version:

Publisher's PDF, also known as Version of record

\section{Please check the document version of this publication:}

- A submitted manuscript is the version of the article upon submission and before peer-review. There can be important differences between the submitted version and the official published version of record.

People interested in the research are advised to contact the author for the final version of the publication, or visit the DOI to the publisher's website.

- The final author version and the galley proof are versions of the publication after peer review.

- The final published version features the final layout of the paper including the volume, issue and page numbers.

Link to publication

\footnotetext{
General rights rights.

- You may freely distribute the URL identifying the publication in the public portal. please follow below link for the End User Agreement:

www.umlib.nl/taverne-license

Take down policy

If you believe that this document breaches copyright please contact us at:

repository@maastrichtuniversity.nl

providing details and we will investigate your claim.
}

Copyright and moral rights for the publications made accessible in the public portal are retained by the authors and/or other copyright owners and it is a condition of accessing publications that users recognise and abide by the legal requirements associated with these

- Users may download and print one copy of any publication from the public portal for the purpose of private study or research.

- You may not further distribute the material or use it for any profit-making activity or commercial gain

If the publication is distributed under the terms of Article $25 \mathrm{fa}$ of the Dutch Copyright Act, indicated by the "Taverne" license above, 


\section{UNDERSTANDING HIV-RELATED STIGMA: SOCIAL AND PSYCHOLOGICAL PROCESSES}
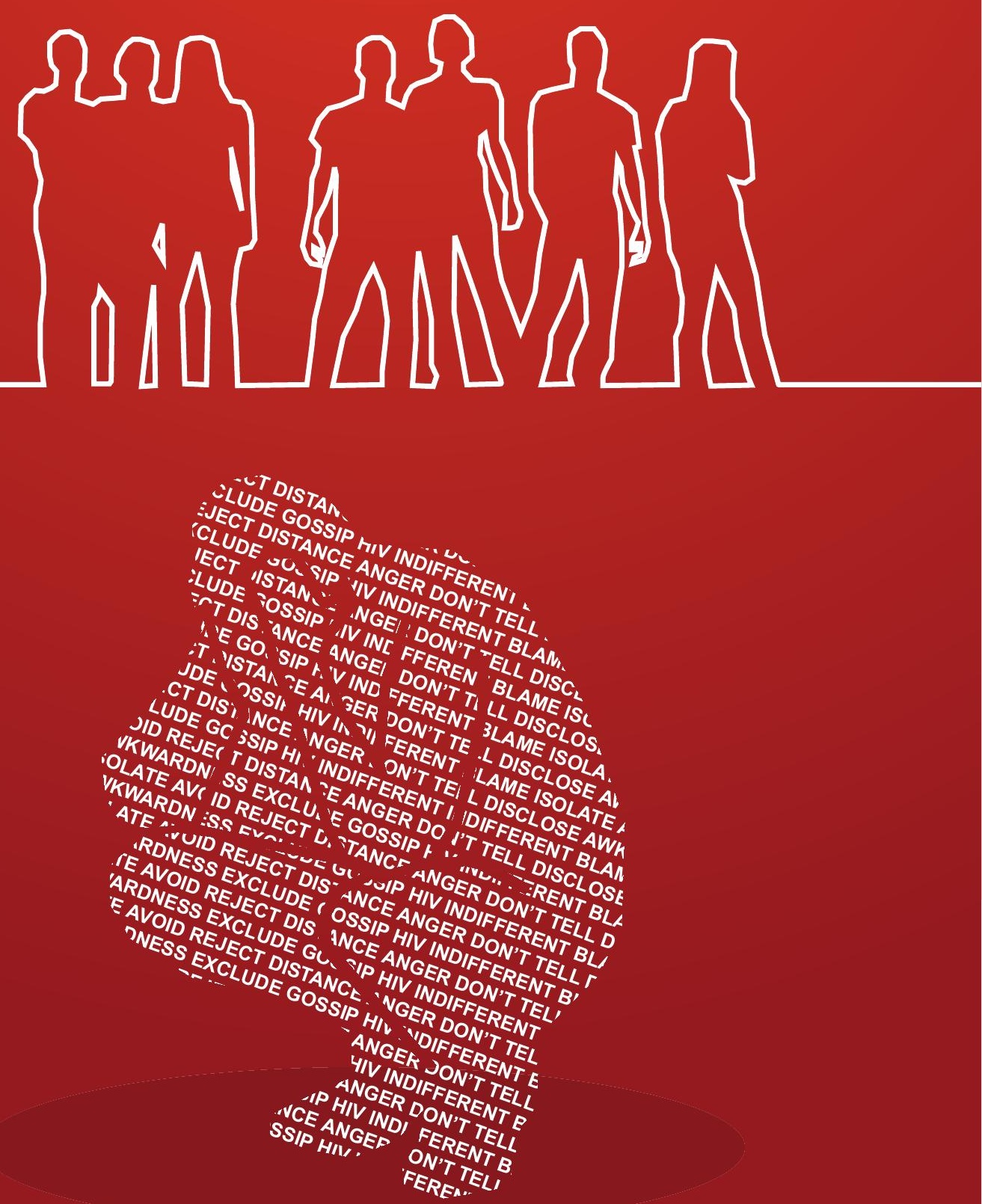

SARAH E. STUTTERHEIM 



\section{UNDERSTANDING HIV-RELATED STIGMA: SOCIAL AND PSYCHOLOGICAL PROCESSES}




\section{Colophon}

(C) 2011, Sarah E. Stutterheim, Maastricht, the Netherlands

Graphic design by Jaring Dijkstra

Printed by F\&N Boekservice, Amsterdam

All rights reserved. No part of this publication may be reproduced, stored in a retrieval system, transmitted in any form, or by any means, electronic, mechanical, photocopying, recording, or otherwise, without the prior written permission of the author.

The research presented in this dissertation was funded by AIDS Fonds and Abbott. Abbott also provided financial support for the printing of this dissertation.
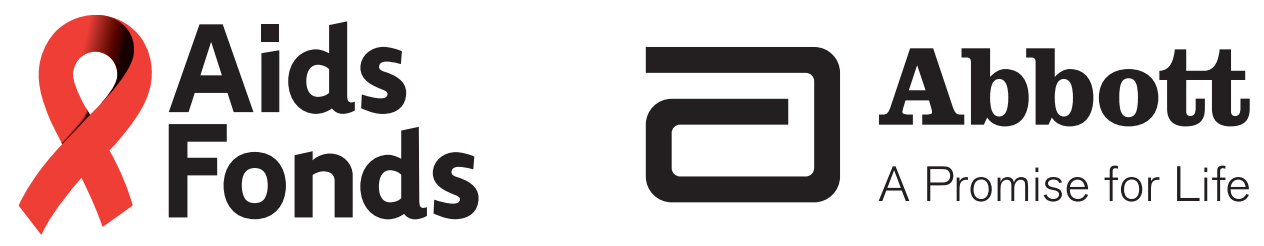


\section{UNDERSTANDING HIV-RELATED STIGMA: SOCIAL AND PSYCHOLOGICAL PROCESSES}

\section{PROEFSCHRIFT}

Ter verkrijging van de graad van doctor aan de Universiteit Maastricht, op gezag van de Rector Magnificus prof. mr. G. P. M. F. Mols, volgens het besluit van het College van Decanen, in het openbaar te verdedigen op vrijdag 11 februari 2011 om 12:00

door

SARAH ELISABETH STUTTERHEIM 


\section{Promotores}

Prof. dr. Gerjo Kok (Universiteit Maastricht)

Prof. dr. John B. Pryor (Illinois State University \& Universiteit Maastricht)

\section{Copromotor}

Dr. Arjan E. R. Bos (Universiteit Maastricht)

\section{Beoordelingscommissie}

Prof. dr. Harm J. Hospers (voorzitter; Universiteit Maastricht)

Dr. Anton J. M. Dijker (Universiteit Maastricht)

Prof. dr. Glenn D. Reeder (Illinois State University)

Prof. dr. Nanne de Vries (Universiteit Maastricht)

Dr. Onno de Zwart (GGD Rotterdam) 
For Herman 



\section{TABLE OF CONTENTS}

CHAPTER 1: $\quad$ Introduction 1

CHAPTER 2: $\quad$ Beliefs contributing to HIV-related stigma in African and Afro-Caribbean communities in the Netherlands

CHAPTER 3:

HIV-related stigma in African and Afro-Caribbean

communities in the Netherlands: Manifestations, consequences, and coping

CHAPTER 4:

HIV status disclosure among HIV-positive African and Afro-Caribbean people in the Netherlands

CHAPTER 5:

HIV-related stigma and psychological distress: The harmful effects of specific stigma manifestations in various social settings

CHAPTER 6:

Psychological and social correlates of HIV status disclosure: The significance of stigma visibility

CHAPTER 7:

General discussion

REFERENCES

SUMMARY 

INTRODUCTION 
As we enter the fourth decade of the HIV epidemic, focus is increasingly being placed on the social context of HIV. More and more attention is being paid to what it means to live with HIV and to the impact of an HIV infection for people living with HIV (PLWH), their families, and their communities. An important issue in this context, and one that contributes significantly to the hidden burden of HIV, is stigma (Weiss, Ramakrishna, \& Somma, 2006).

The term stigma dates back to the Greeks who cut or burned marks into the skin of criminals, slaves, and traitors in order to identify them as tainted or immoral people that should be avoided (Goffman, 1963). As we know it today, stigma is not merely a physical mark but rather an attribute that results in widespread social disapproval. Sociologist Erving Goffman (1963) defined stigma as a discrediting social difference that yields devaluation or a 'spoiled social identity.' According to Goffman (1963), stigma is inherently rooted in social interactions. Through the 'language of relationships,' what is 'abnormal' or deviant is determined in the context of what is 'normal' or expected, and vice versa.

Since Goffman, the concept of stigma has been expanded and adapted by the field of social psychology. Social psychologists see stigma as comprising two fundamental components, namely the recognition of difference and devaluation (Dovidio, Major, \& Crocker, 2000). According to Crocker and colleagues (1998), "stigmatized individuals possess (or are believed to possess) some attribute, or characteristic, that conveys a social identity that is devalued in a particular social context" (p. 505). Also in line with Goffman, social psychologists have placed emphasis on how stigma arises in social interactions. Stigma is thus considered to be a socially constructed attribute that does not reside in the person but rather in the social context (Alonzo \& Reynolds, 1995; Crocker, Major, \& Steele, 1998; Herek, Capitanio, \& Widaman, 2002; Jones et al., 1984; Major \& O'Brien, 2005). What is stigmatizing in one social context may not be stigmatizing in another.

In most social psychological studies, emphasis has been placed on the cognitive origins of stigma - thus the perceptions, beliefs, or attitudes of those who stigmatize others - and the consequences of said perceptions for social interaction (Campbell \& Deacon, 2006; Mahajan et al., 2008). As such, much of the focus has been placed on the individual perceiver (i.e. the person that does the stigmatizing; Dovidio, Major, \& Crocker, 2000; Weiss, Ramakrishna, \& Somma, 2006). This micro-level, individualistic approach to studying stigma has been criticized by sociologists who contend that more attention should be paid to how structural factors promote and maintain stigma (Link \& Phelan, 2001; Parker \& Aggleton, 2003). Consequently, recent literature on stigma tends to acknowledge that stigma reproduces existing social inequalities and is perpetuated by hegemony and 
the exercise of social, economic, and political power (Campbell \& Deacon, 2006; Rankin, Brennan, Schell, Laviwa, \& Rankin, 2005; Scambler \& Paoli, 2008). The recent literature on stigma is also characterized by a shift in emphasis from the perceiver to the target of stigma. In an effort to balance the vast amount of literature on the psychological processes that cause people to stigmatize others, we have seen a proliferation of studies investigating how stigmatized individuals, their families, and their communities experience, are impacted by, and cope with stigma.

\section{CATEGORIZING STIGMAS}

Goffman (1963) delineated three stigma categories: tribal stigmas, abominations of the body, and blemishes of individual character. The first is based on membership in a devalued group (e.g. race, religion), the second on physical characteristics (e.g. facial disfigurement, physical disabilities), and the third on devalued personal characteristics (e.g. addiction, unemployment). Since Goffman, numerous ways of classifying stigmas have been developed. Some focus on how stigma is expressed (e.g. Scambler \& Hopkins, 1986) while others categorize according to influencing features (e.g. Dijker \& Koomen, 2003; Jones et al., 1984).

With respect categories based on how stigma is expressed, Pryor and Reeder (in press) recently developed a taxonomy that seeks to bring greater conceptual clarity to the current but diverse literature on stigma. In their view, there are four types of stigma (see Figure 1.1). The first is public stigma. Public stigma is at the core of Pryor and Reeder's model and represents people's social and psychological reactions to someone they perceive to have a stigmatizing condition. Public stigma comprises cognitive (stereotypes), affective (prejudice), and behavioral (discrimination) components and reflects the perspective of the perceiver. The behavioral component of public stigma, namely discriminatory acts, is likely analogous to what Scrambler and Hopkins (1986) termed enacted stigma. The second type of stigma in Pryor and Reeder's model is self stigma. Self stigma reflects the social and psychological impact of possessing a stigma. Self stigma is similar to what has previously been termed felt stigma (Scambler \& Hopkins, 1986), perceived stigma (Bond, Chase, \& Aggleton, 2002), or anticipated stigma (Earnshaw \& Chaudoir, 2009), and includes both a fear of being exposed to stigmatization and the potential internalization of the negative beliefs and feelings associated with the stigmatized condition (internalized stigma). The third type of stigma is stigma-by-association. Stigma-by-association, a term originally coined by Neuberg and colleagues (1994), is analogous to Goffman's (1963) courtesy stigma and entails social and psychological reactions to people associated with 
a stigmatized person (e.g. family and friends). Pryor and Reeder also include the impact of being connected to a stigmatized person in the concept of stigma-by-association. Thus, their conceptualization of this construct has analogues to both public stigma and selfstigma. The final type of stigma delineated by Pryor and Reeder is institutional stigma. Institutional stigma is defined as the "legitimatization and perpetuation of a stigmatized status by society's institutions and ideological systems" (p. 4) and reflects the previously mentioned growing trend toward acknowledging structural factors and the role of power and dominance in stigmatization processes.

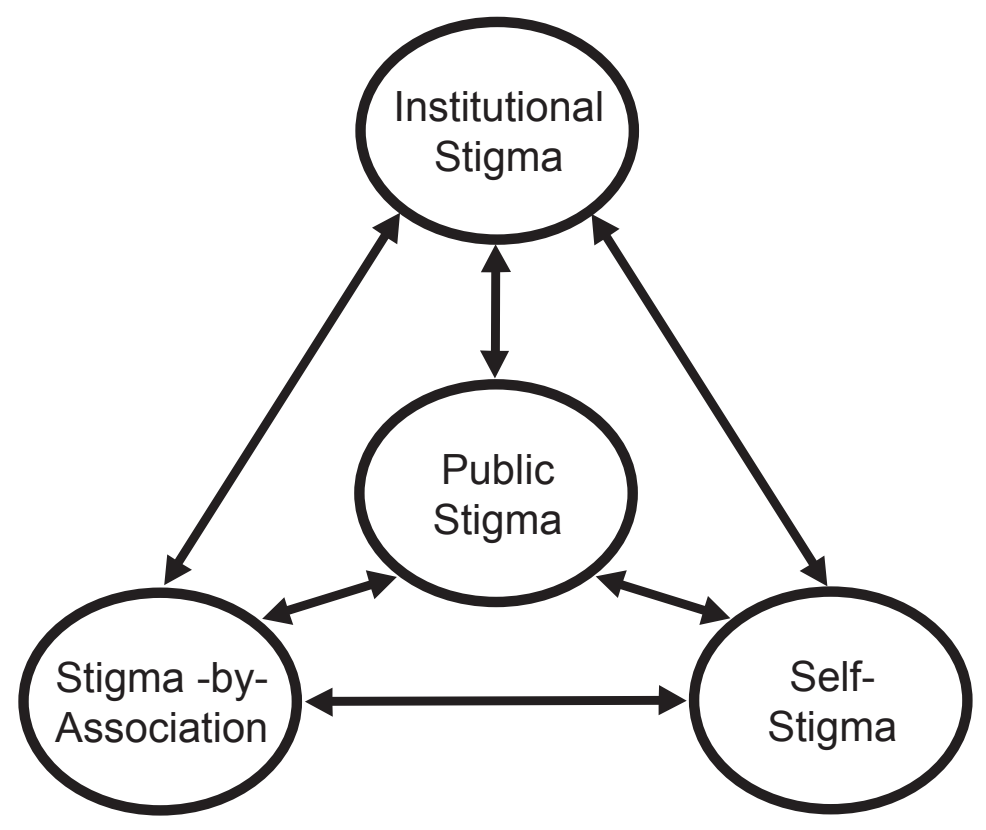

\section{Figure 1.1: Four types of stigma}

Source: Pryor and Reeder (in press)

Taxonomies that classify stigmas according to the features that influence the degree to which one is stigmatized tend to focus on a few key features. For example, Jones and colleagues (1984) delineated five dimensions of stigma, namely concealability, disruptiveness, aesthetic qualities, peril, and origin. In Jones et al.'s view, if a stigmatized condition is visible, disrupts or hampers social interactions, is unattractive, is considered dangerous, and if the possessor of the stigma is perceived to be personally responsible for its acquisition, stigmatization is most likely. Later, Dijker and Koomen (2003), in their 
extension of Weiner's (1993) attribution-emotional model of stigmatization, outlined three features of stigma (seriousness, responsibility, and negative valence of behavioral cause) and their impact on emotions (anxiety, [lack of] pity, and irritation) that can contribute to stigmatization. Bos, Schaalma, and Pryor (2008) have since published an adapted version of this model (see Figure 1.2). In their adaptation, cognitions concerning four features of a condition lead to emotions that, in turn, can yield stigmatization. The four features are contagiousness, seriousness, personal responsibility, and norm-violating behavior and the posited emotions are fear, anger, and (lack of) pity. Fear is promoted by high levels of perceived contagiousness and perceived seriousness. Anger is promoted by attributions of personal responsibility and associations between the condition and norm-violating behavior. Lastly, pity or compassion for a person with a stigmatized condition is promoted by high levels of perceived seriousness but inhibited by attributions of personal responsibility and associations with norm-violating behavior. Thus, when a condition is considered highly contagious and very severe, when the person with that condition is considered personally responsible for its origin, and when the condition is associated with behaviors that are considered to violate the norms of the majority, stigmatization is highly likely.

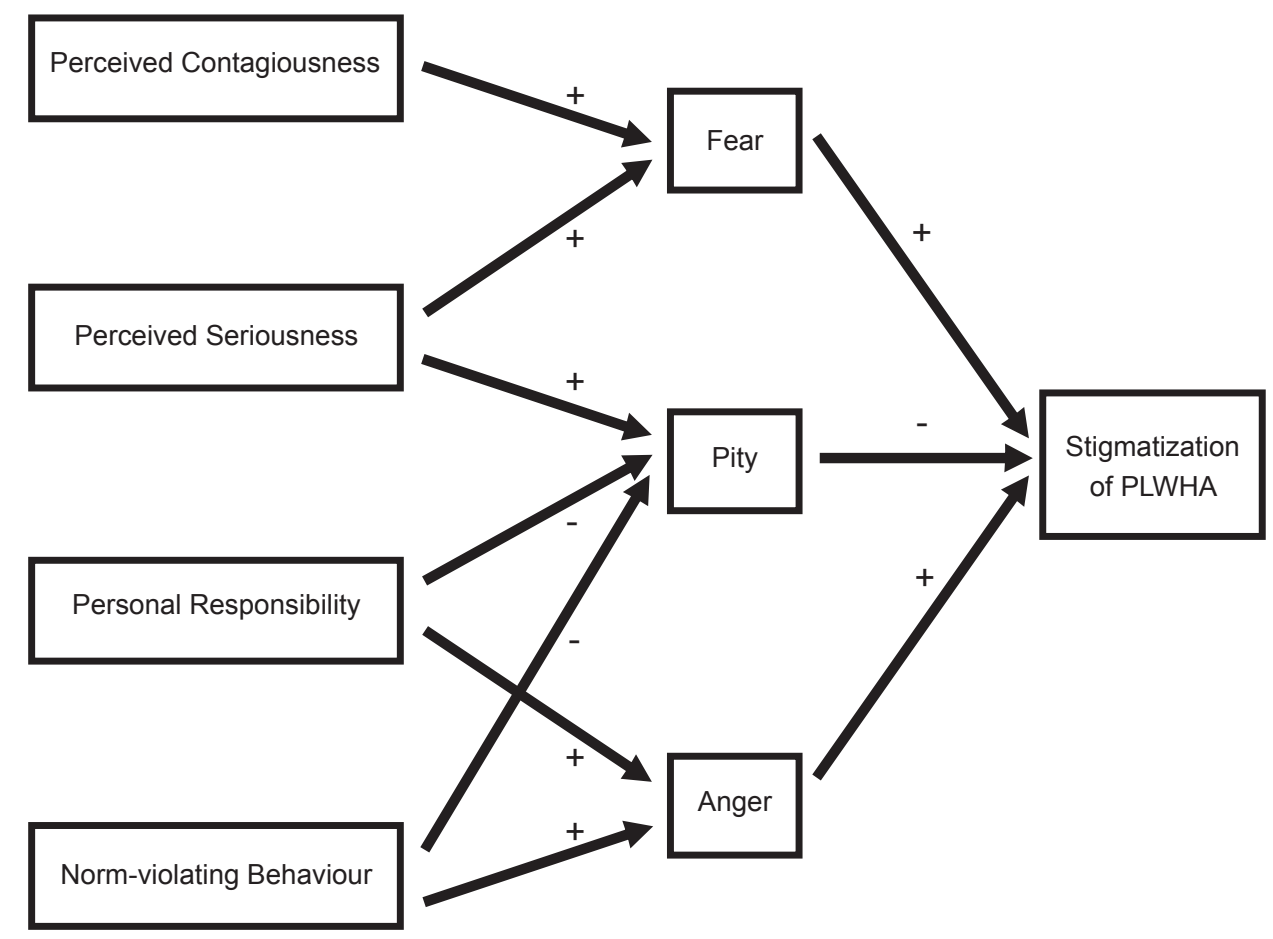

Figure 1.2: Cognitive-emotional model of HIV-related stigmatization

Source: Bos, Schaalma, and Pryor (2008) 


\section{HIV-RELATED STIGMA}

Few modern illnesses have been as extensively stigmatized as HIV (Black \& Miles, 2002) and when we consider the above mentioned features that contribute to stigma, this is not surprising. Despite extensive health education campaigns, a fear of acquiring HIV through casual social interaction remains, as does the association of HIV with death and wasting. Additionally, because HIV is a condition that is often acquired through volitional behavior, many consider PLWH personally responsible for having HIV or view HIV as due justice for reckless or immoral behavior. Lastly, HIV has, since its inception, been associated with behaviors viewed by many as socially unacceptable such as homosexuality, intravenous drug use, and commercial sex work (Alonzo \& Reynolds, 1995; Bos, Kok, \& Dijker, 2001; Malcolm et al., 1998; Pryor, Reeder, \& Landau, 1999). Together, these perceptions contribute to HIV being a powerfully stigmatizing condition.

HIV-related stigma affects the treatment of people living with HIV (PLWH) in a number of ways and across a broad range of settings. Manifestations of stigma include avoidance, exclusion, rejection, isolation, social ostracism, blaming, violence, service denial, physical distance, indifference, awkward social interaction, and being advised to conceal one's status. Relevant settings in which stigmatization can occur are with families, in communities, among friends and acquaintances, with sexual partners, in health care settings, with respect to housing, in the financial services sector, within religious institutions, at work, while travelling or migrating, and in educational settings (Greeff et al., 2008; Malcolm et al., 1998; Shamos, Hartwig, \& Zindela, 2009; Stutterheim et al., 2009; Varas-Diaz, SerranoGarcia, \& Toro-Alfonso, 2005). HIV-related stigma can also be felt indirectly when, for example, PLWH hear others talk negatively about HIV. In such cases, PLWH are not the targets of active discrimination but are exposed to the endorsement or acceptance of discrimination (Black \& Miles, 2002; Steward et al., 2008; Weiss, Ramakrishna, \& Somma, 2006). Because PLWH are often acutely aware of the public stigma surrounding HIV, the impact of stigma can also come from an anticipation of negative reactions from others if their condition is known. This anticipation can cause PLWH to live in secrecy and constantly be concerned about their condition being revealed.

The consequences of HIV-related stigma are severe and can impact not only PLWH but also their families and communities. HIV-related stigma has been found to hamper HIV prevention efforts (UNAIDS, 2008), inhibit treatment adherence (Chesney \& Smith, 1999; Nyblade, Pande, Mathur, MacQuarrie, \& Kidd, 2003), and function as a barrier to HIV testing (Meiberg, Bos, Onya, \& Schaalma, 2008; Vermeer, Bos, Mbwambo, Kaaya, \& Schaalma, 2009). It also negatively impacts social interactions between PLWH and others, 
and can result in decreased social network size, limited social support, and social isolation (J. D. Lee \& Craft, 2002; Lichtenstein, Laska, \& Clair, 2002). Psychologically, HIV-related stigma can generate significant distress in the form of depression, anxiety, and lowered self esteem (R. S. Lee, Kochman, \& Sikkema, 2002; Stutterheim et al., 2009; Vanable, Carey, Blair, \& Littlewood, 2006). Clearly, the negative consequences of stigmatization toward PLWH are substantial.

PLWH can attempt to mitigate the negative psychological and social impact of HIV-related stigma by employing coping strategies. Some coping strategies are geared to altering the relationship between PLWH and their environment. These strategies are called problem-focused coping strategies. Other strategies seek to regulate negative emotions and are called emotion-focused coping strategies. Problem-focused coping strategies can target the self, the situation, or others, and include strategies such as selective disclosure, compensating for the stigma during social interactions, avoiding situations where stigmatization is likely (i.e. disengagement), affiliating oneself with similar others, seeking social support, and activism. Emotion-focused strategies include downward social comparison, external attributions, denial or prejudice minimization, distraction, positive reappraisal, and disidentification with the stigmatized identity (Crocker, Major, \& Steele, 1998; Major \& O’Brien, 2005; Medley, Kennedy, Lunyolo, \& Sweat, 2009; Miller \& Kaiser, 2001). Understanding which coping strategies PLWH employ is particularly important because some coping strategies mitigate the negative consequences of HIV-related stigma better than others do. For example, coping strategies such as support seeking and positive reappraisal have been found to be positively related to psychological well-being while stigma avoidance has been found to yield greater psychological distress (Gonzalez, Solomon, Zvolensky, \& Miller, 2009; Kraaij et al., 2008).

\section{DISCLOSURE OF HIV STATUS}

Given the negative social and psychological consequences of HIV-related stigma for PLWH, one might ask why PLWH would choose to disclose their status. Even if one can cope adequately with stigmatizing reactions from others, would it not just be wiser to keep one's status a secret? There is a substantial body of literature that shows that PLWH do face a dilemma when it comes to disclosure (Pachankis, 2007). On the one hand, disclosure can lead to stigmatization (Black \& Miles, 2002; Landau \& York, 2004) which, as mentioned above, can be detrimental to their social lives and psychological well-being (Riggs, Vosvick, \& Stallings, 2007; Stutterheim et al., 2009; Vanable, Carey, Blair, \& Littlewood, 2006). On the other, disclosure can be beneficial. It has been found to promote treatment 
adherence (Chesney \& Smith, 1999), safe sex (Melchert \& Patterson, 1999; Serovich \& Mosack, 2003), psychological well-being (Derlega, Winstead, Oldfield, \& Barbee, 2003; Smart \& Wegner, 1999), closeness in relationships (Herek \& Capitanio, 1996; Parsons, VanOra, Missildine, Purcell, \& Gomez, 2004), and social support provision (Bos, Kanner, Muris, Janssen, \& Mayer, 2009; Smith, Rossetto, \& Peterson, 2008). Clearly, there are both advantages and disadvantages to disclosure. Perhaps the greatest advantage is that disclosure can lead to social support. Social support not only enables PLWH to better cope with health concerns (Smith, Rossetto, \& Peterson, 2008) but also buffers stress, anxiety, and depression (Kalichman, DiMarco, Austin, Luke, \& DiFonzo, 2003; Lam, NaarKing, \& Wright, 2007; Li, Lee, Thammawijaya, Jiraphongsa, \& Rotheram-Borus, 2009). However, it is clear that the very disclosure that can generate social support can also yield stigmatization. PLWH must therefore take the risk of being met with stigmatizing reactions in order to gain the support necessary to deal with stigmatizing reactions.

What determines whether one discloses or conceals one's HIV status? Previous research has contended that HIV status disclosure is a reasoned process whereby the perceived costs and benefits to oneself and to others are weighed. When PLWH consider the benefits to outweigh the costs, disclosure is highly probable. When the costs outweigh the benefits, concealment is more likely (Black \& Miles, 2002; Derlega, Winstead, Greene, Serovich, \& Elwood, 2004; Serovich, 2001; Valle \& Levy, 2009). In some cases, disclosure is not a choice and PLWH are not in a position to, as Goffman (1963) would say, 'pass' as 'normal'. Disease progression and, more frequently, side effects of highly active antiretroviral therapy (HAART), such as lipodystrophy syndrome, can make HIV a condition with conspicuous symptoms. In such cases, PLWH do not have a choice. They are 'outed' by their appearance. Some research on the psychological implications of concealable versus visible stigmas has demonstrated that people with concealable stigmas experience more anxiety, depression, and negative affect, as well as lower self esteem, than people with visible stigmas (Frable, Platt, \& Hoey, 1998), thus suggesting that PLWH with visible symptoms are likely better off. This is perhaps because they can access and employ important coping strategies that are not as readily available to PLWH who hide their status (Quinn, 2006). For example, people with visible stigmas are often in a better position to find and compare themselves to in-group members, and they might more readily attribute negative treatment to prejudice (Crocker, Major, \& Steele, 1998). At the same time, research specific to HIV has demonstrated a significant relationship between visible symptoms and psychological distress (Reynolds, Neidig, Wu, Gifford, \& Holmes, 2006; Sanches, Mill, Machado, Donadi, \& Morais Fernandes, 2009). It is possible that this relationship is due to the fact that PLWH with visible symptoms experience higher levels 
of stigma than PLWH who can conceal their status, or because they have less control over how disclosure takes place. Bos, Dijker and Koomen (2007) have previously shown that the way in which disclosure occurs can impact how targets of disclosure respond to the disclosure. Positive reactions to disclosure can be promoted by selecting the optimal setting, person, and time, and that is something that PLWH with visible symptoms are often unable to do. Their disclosure is less likely to be voluntary (Joachim \& Acorn, 2000) and may therefore be met with less understanding and support.

\section{HIV-RELATED STIGMA IN THE AFRICAN AND AFRO-CARIBBEAN DIASPORA}

Ethnic minorities in the developed world, and particularly African and Afro-Caribbean diaspora, are not only disproportionately affected by the HIV epidemic in terms of prevalence; they are also particularly vulnerable to HIV-related stigma.

Sub-Saharan Africa and the Caribbean have long had the highest HIV prevalence rates in the world (UNAIDS, 2009). The generalized epidemics in these regions have consequently yielded high prevalence rates in African and Afro-Caribbean diaspora communities. In the Netherlands, non-Western migrants comprise one tenth of the Dutch population but one third of all HIV cases. Of that third, more than half originate from Sub-Saharan Africa and almost a third from the Caribbean (Shiripinda \& van Eerdewijk, 2008). In terms of the total PLWH population in the Netherlands, one in every five is of African or Caribbean origin (HIV Monitoring Foundation, 2008).

Not only are prevalence rates for HIV higher in these diaspora communities, so too is HIV-related stigma. High levels of stigmatization have been reported not only in SubSaharan Africa (Greeff et al., 2008; Kalichman \& Simbayi, 2004; Nyblade, Pande, Mathur, MacQuarrie, \& Kidd, 2003) and the Caribbean (Carr, 2004; Varas-Diaz, Serrano-Garcia, \& Toro-Alfonso, 2005) but also in these regions' diaspora communities in the developed world (M. Anderson et al., 2008; Dodds, 2006; Gardezi et al., 2008). The fear of stigmatization and the degree to which stigmatization is experienced have also been reported to be substantially greater among black PLWH living in the developed world than among white PLWH (Dodds, 2006; Erwin, Morgan, Britten, Gray, \& Peters, 2002). This may, at least in part, be because African and Afro-Caribbean diaspora are already socially marginalized and disadvantaged by racism, immigration processes, and anti-asylum discourses, and experience barriers in accessing health and social services as well as employment (Dodds, 2006; Dodds et al., 2004; Shiripinda \& van Eerdewijk, 2008). As such, their HIV-related stigma is compounded by other socially stigmatized conditions thus resulting in what is 
termed layered or multiple stigmatization (L. Brown, Macintyre, \& Trujillo, 2003; Deacon, 2006; Genberg et al., 2009; Reidpath \& Chan, 2005).

The culture in African and Afro-Caribbean PLWH's home countries, and in their diaspora communities, may also influence the experience and impact of HIV-related stigma. HIVrelated stigma is considered to be universal but, at the same time, it is characterized by cross-cultural diversity and complexity (Parker \& Aggleton, 2003; Stangor \& Crandall, 2000). The nature of HIV-related stigma, how it manifests, its impact, and the perceptions or beliefs that drive it are thought to vary from one culture to another and from one community to another (Deacon, 2006; Maman et al., 2009; Norman, Abreu, Candelaria, \& Sala, 2009; Pryor, Reeder, \& Landau, 1999; Steward et al., 2008). Disclosure processes and coping mechanisms are also thought to vary across cultures (Chandra, Deepthivarma, \& Manjula, 2003; Greeff et al., 2008; Tate, Van Den Berg, Hansen, Kochman, \& Sikkema, 2006). As such, scholars have contended that HIV-related stigma must be considered within its specific social and cultural context (Bos, Schaalma, \& Pryor, 2008; Deacon, Stephney, \& Prosalendis, 2005; Trickett, 2009; Visser, Makin, \& Lehobye, 2006).

To date, research on how African and Afro-Caribbean PLWH in diaspora communities in the developed world experience, are impacted by, and cope with stigmatization is limited. Additionally, little is known regarding their disclosure processes. Further, there is a paucity of literature on the perceptions that underlie and drive the stigmatization of PLWH in African and Afro-Caribbean diaspora communities. The studies that have been conducted have occurred almost exclusively in the United Kingdom thus suggesting that we, to date, simply know too little about HIV-related stigma in African and Afro-Caribbean diaspora communities.

\section{OUTLINE OF THIS DISSERTATION}

This dissertation reports on the social and psychological processes involved in the production and experience of HIV-related stigma. It explores public and self stigma and follows recent trends in stigma research by focusing predominantly, but not exclusively, on the perspective of PLWH as this can enable us to better "appreciate differences in sociocultural worldviews underlying differences in cognitive construals and the implications of these differences for the perceptions of prejudice, the coping mechanism selected, and the psychological consequences of prejudice" (Oyserman \& Swim, 2002, p. 3).

The first part of this dissertation describes the results of comprehensive qualitative research conducted with African, Dutch Antillean, and Surinamese diaspora in the Netherlands. This 
section focuses on both the perceiver and the target of stigma by following the process of stigmatization from the perceiver's beliefs regarding HIV and PLWH to the subsequent manifestations of stigma, the consequences for the target, and how PLWH cope with stigma and determine whether or not to disclose to others. As such, Chapter 2 explores the beliefs that underlie and contribute to HIV-related stigma in these diaspora communities from the perspective of both HIV-positive and HIV-negative community members. Chapter 3 , again from the perspective of both HIV-positive and HIV-negative diaspora community members, describes the manifestations and consequences of HIV-related stigma for PLWH, and delineates the coping strategies employed by PLWH to mitigate the negative social and psychological consequences of HIV-related stigma. Chapter 4 focuses on how African and Afro-Caribbean PLWH approach disclosure. In particular, it investigates their reasons for and against disclosure of HIV status.

The second part of this dissertation explores some of the consequences of HIV-related stigma in more detail and expands the study population and sample to include all PLWH living in the Netherlands. The findings reported in this section are, in contrast to the first part of this dissertation, quantitative in nature. Chapter 5 investigates, using a cross-sectional survey, which specific stigma experiences most strongly predict psychological distress across a number of social settings while Chapter 6 explores the psychological and social consequences of visible versus concealable stigmas. More specifically, it investigates HIV-related stigma, psychological distress, self esteem, and social support in a sample comprising people who have concealed their HIV status to all but a selected few (limited disclosers), people who can conceal but chose to be open (full disclosers), and people who have visible symptoms that make concealing difficult (visibly stigmatized).

The final chapter of this dissertation, Chapter 7 , is a general discussion of all research findings, both the qualitative findings acquired through research with African, Dutch Antillean, and Surinamese diaspora communities and the quantitative findings gathered through cross-sectional research with the general PLWH population in the Netherlands. This chapter not only summarizes the findings of the studies reported in this dissertation and discusses them in the context of the current literature; it also discusses the role of culture in understanding stigma, shortly reflects on the methods used to conduct the research reported in this dissertation, outlines implications of the research findings, and provides recommendations for future research. 
INTRODUCTION

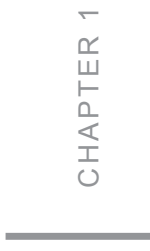




\section{BELIEFS CONTRIBUTING TO HIV-RELATED STIGMA IN \\ AFRICAN AND AFRO-CARIBBEAN COMMUNITIES IN THE NETHERLANDS}

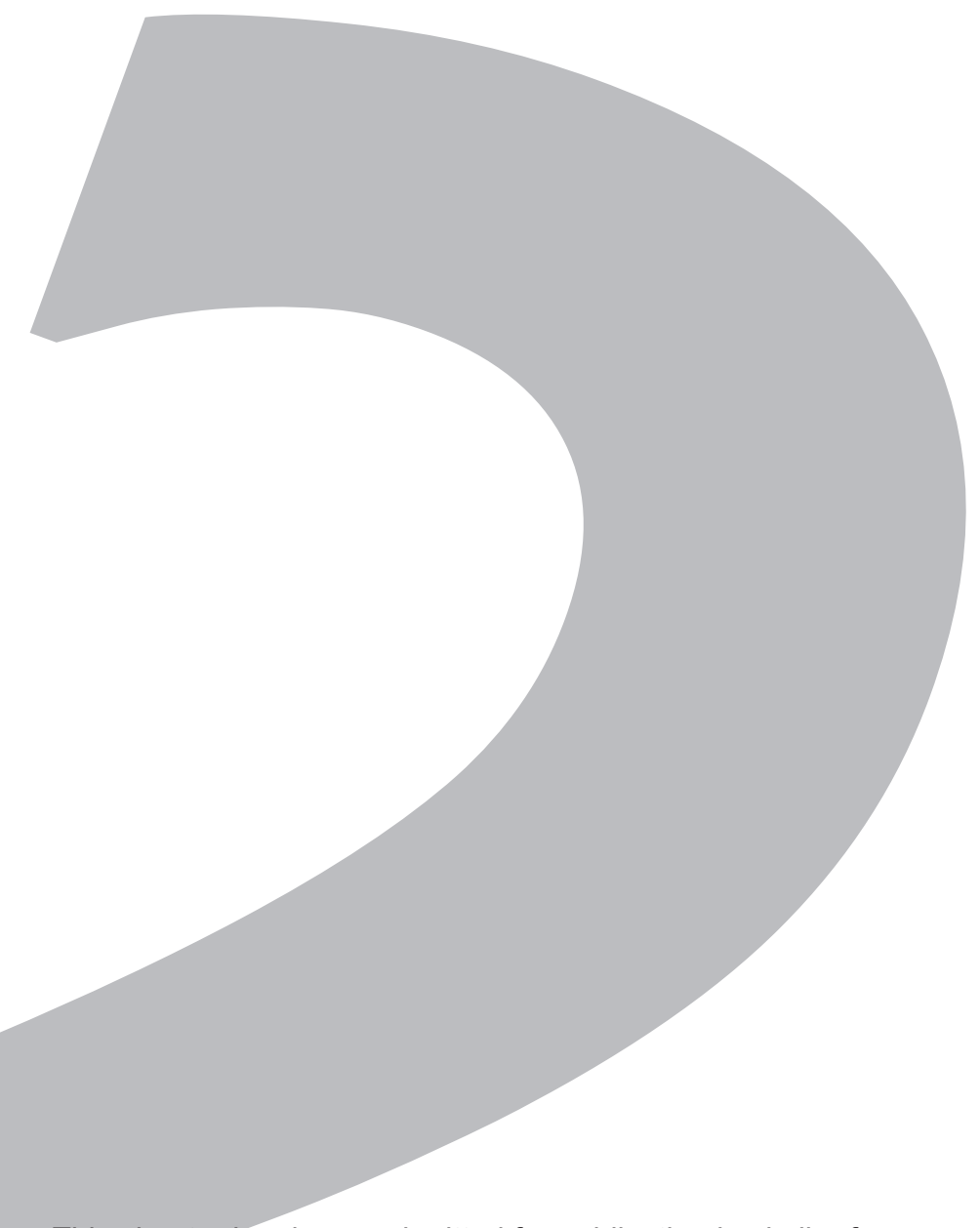

This chapter has been submitted for publication in similar form as:

Stutterheim, S. E., Bos, A. E. R., van Kesteren, N. M. C., Shiripinda, I., Pryor, J. P., de Bruin, M., \& Schaalma, H. P. Beliefs contributing to HIV-related stigma in African and Afro-Caribbean communities in the Netherlands. 


\section{ABSTRACT}

Thirty years after the first diagnosis, people living with HIV (PLWH) around the world continue to report stigmatizing experiences. In this study, beliefs contributing to HIVrelated stigma in African and Afro-Caribbean diaspora communities and their cultural context were explored through semistructured interviews with HIV-positive $(N=42)$ and HIV-negative $(N=52)$ African, Antillean, and Surinamese diaspora community members in the Netherlands. Beliefs that HIV is highly contagious, that HIV is a very severe disease, and that PLWH are personally responsible for acquiring their HIV infection were found to contribute to HIV-related stigma, as did the belief that PLWH are HIV-positive because they engaged in norm-violating behavior such as promiscuity, commercial sex work, and, for Afro-Caribbean diaspora, also homosexuality. These beliefs were found to be exacerbated and perpetuated by cultural taboos on talking about HIV and sexuality. HIV-related stigma reduction interventions should focus on changing these beliefs and breaking cultural taboos on HIV and sexuality in a manner that is participatory and consistent with current theory and empirical findings. 


\section{INTRODUCTION}

Thirty years after the first diagnosis, HIV remains a highly stigmatized condition. Globally, people living with HIV $(\mathrm{PLWH})$ continue to report stigmatizing experiences such as avoidance, abandonment, exclusion, rejection, and blaming (Malcolm et al., 1998; Nyblade, Pande, Mathur, MacQuarrie, \& Kidd, 2003; Stutterheim et al., 2009). The consequences of HIV-related stigma are substantial and include hampered HIV prevention, testing delays, poor treatment adherence, psychological distress in PLWH, and disrupted social interactions (Bos, Kok, \& Dijker, 2001; Mills, 2006; Stutterheim et al., 2009; Vermeer, Bos, Mbwambo, Kaaya, \& Schaalma, 2009). HIV-related stigma is a complex process of devaluation whereby a person is considered to possess a discrediting attribute and is subsequently deemed flawed by others (Crocker, Major, \& Steele, 1998; Goffman, 1963; Jones et al., 1984). Stigma is embedded in community processes and reinforces existing social inequalities and previously defined boundaries between 'us' and 'them' (Campbell \& Deacon, 2006; Nyblade, Pande, Mathur, MacQuarrie, \& Kidd, 2003; Parker \& Aggleton, 2003).

Sub-Saharan Africa and the Caribbean have long had the highest HIV prevalence rates in the world (UNAIDS, 2008). The generalized epidemics in these countries have also yielded high prevalence rates within diaspora communities. In the Netherlands, nonWestern migrants comprise one tenth of the Dutch population but one third of all HIV cases. Of that third, more than half originate from Sub-Saharan Africa and almost a third from the Caribbean (Shiripinda \& van Eerdewijk, 2008). Research has shown that African and Afro-Caribbean PLWH are subjected to extensive stigmatization both in their home countries (Carr, 2004; Nyblade, Pande, Mathur, MacQuarrie, \& Kidd, 2003; Varas-Diaz, Serrano-Garcia, \& Toro-Alfonso, 2005) and in their diaspora communities (M. Anderson et al., 2008; Dodds, 2006).

Research conducted in North America and Europe has shown that a number of beliefs can contribute to HIV-related stigma. The first concerns the contagiousness of HIV. When HIV is considered highly contagious, fear of PLWH and subsequent stigmatization is likely. The second belief pertains to the severity of HIV. When HIV is considered a very serious or fatal condition, fear and stigmatization often result. The third belief concerns responsibility. When PLWH are considered personally responsible for their infection, anger, a lack of compassion, and stigmatization are likely. The fourth belief is that PLWH are HIV-positive because they engaged in norm-violating behavior such as homosexuality, commercial sex work, and intravenous drug use. This belief can also generate anger, a lack of compassion, 
and stigmatization (Bos, Dijker, \& Koomen, 2007; Dijker \& Koomen, 2003; Herek, 1999). In short, when HIV is considered highly contagious and very severe, when PLWH are considered responsible for acquiring HIV, and when HIV is associated with norm-violating behaviors, stigmatization is most likely.

Given that HIV-related stigma is socially constructed and considered to vary from one culture to another and from one community to another (Deacon, 2006; Maman et al., 2009; Norman, Abreu, Candelaria, \& Sala, 2009; Pryor, Reeder, \& Landau, 1999), we believe that it is important to investigate whether the beliefs found to contribute to the stigmatization of PLWH indigenous to developed countries are also held by people and communities in developing nations and in their respective diaspora communities in the developed world. We also believe that it is important to understand the social and cultural context in which such beliefs exist. Consequently, the present study qualitatively explored which beliefs held by African, Antillean, and Surinamese diaspora contribute to the stigmatization of PLWH in their communities and the cultural context of these beliefs. We considered it particularly important to explore these beliefs in these diaspora communities not only because they are disproportionately affected by the epidemic but also because there is a paucity of literature on HIV-related stigma in African and Afro-Caribbean diaspora communities in the developed world. In fact, to our knowledge, no study has comprehensively explored the beliefs contributing to HIV-related stigma in African and Afro-Caribbean diaspora communities. A few studies have touched on HIV's association with death, immorality, and promiscuity, and the fact that there is significant fear of contamination in these diaspora communities (M. Anderson et al., 2008; Burns, Imrie, Nazroo, Johnson, \& Fenton, 2007; Dodds et al., 2004; Gardezi et al., 2008) but none have explored the beliefs contributing to HIV-related stigma extensively.

\section{METHODS}

Following study approval by Maastricht University's Ethics Committee, members of African, Dutch Antillean, and Surinamese communities were recruited for face-toface, semistructured interviews by a researcher (SS, IS, MB) or by one of twelve peer interviewers employed and trained by the researchers. Recruitment was purposeful and occurred directly through interviewers or through announcements distributed by the Dutch HIV Association, Humanitas Foundation, or HIV nurses working in Dutch hospitals. Both HIV-positive and HIV-negative African, Antillean, and Surinamese community members were included in the study as this allowed for triangulation across data sources. Our sample comprised 42 HIV-positive participants, of which 16 were African, 9 Antillean, 16 
Surinamese, and 1 both Antillean and Surinamese, and 52 HIV-negative participants, of which 16 were African, 19 Antillean, and 17 Surinamese. Once recruited, participants were given information regarding the study's purpose and procedure. Informed consent was obtained and a monetary reward of $€ 30$ was provided after interview completion. The interviews were held between January 2005 and May 2008 and were guided by a structured protocol of open-ended questions with follow-up probes. The protocol explored: 1) what HIV means to African and Afro-Caribbean diaspora; 2) how HIV is viewed by African and Afro-Caribbean diaspora communities; and 3) how PLWH are viewed by African and AfroCaribbean diaspora communities.

To enhance rigor, all interviews were recorded with a digital voice recorder and transcribed verbatim. Data were processed with QSR NVivo 2.0 and analyzed using a general inductive approach. Each transcript was read thoroughly while listening to the corresponding recording to identify emerging themes and establish categories to which text fragments were assigned. As coding occurred, categories and subcategories were linked to one another. A decision trail was maintained. All emergent categories were documented, as were changes made to the categories and the rationale for those changes. Coding continued until saturation was evident. Findings were subsequently checked with relevant stakeholders in the African, Antillean, and Surinamese communities in the Netherlands.

\section{RESULTS}

\section{Perceived contagiousness}

African, Antillean, and Surinamese participants, both HIV-positive and HIV-negative, reported that people in their community tend to believe that HIV is easily acquired and transmitted through casual contact such as touching, shaking hands, sharing eating and drinking utensils, and, in some cases, simply being in the same space as PLWH. One participant said, "People think that if you spend time with someone who has HIV, you will get it too, like if you touch that person, you'll automatically be infected" (HIV-negative Surinamese woman). Another stated, "There are still people who believe that you can get contaminated by sharing eating utensils and drinking utensils and clothes, and things like that" (HIV-negative South African woman).

The link between HIV and contagiousness was also apparent in HIV-negative community members' descriptions of PLWH. Particularly Antillean and Surinamese participants reported that PLWH are considered "dirty". One participant said, "[PLWH] are talked about very negatively ... That they are dirty or something" (HIV-negative Surinamese woman). 
Another said, "If someone has something you don't want to get, you can almost call it dirty. No one will want to be around that person" (HIV-negative Antillean woman).

That HIV is perceived as highly contagious in African, Antillean, and Surinamese communities was confirmed by HIV-positive participants: "They think if they touch you, they'll automatically get HIV, or if they talk to you - that's what they think - or even eating from the same plate" (HIV-positive Surinamese woman). Another HIV-positive participant said, "People are more afraid to have any kind of contact with you even though it is commonly known that people cannot get HIV through just hugging or drinking from the same glass" (HIV-positive Congolese man).

Many participants, both HIV-negative and HIV-positive, attributed high levels of perceived contagiousness and consequent fear of PLWH to ignorance, as exemplified by the citation below.

People are not aware of the virus and how you can get infected. People think that if they come in contact with that person, shake a hand, they will get it but that's not it. It's just that people don't know enough about the disease. People think that if they touch someone with HIV, they will become infected but that isn't true. I think it's ignorance. (HIV-negative Antillean woman)

Interestingly, this proclaimed lack of knowledge was not apparent among this study's HIVnegative African, Antillean, and Surinamese participants. A few participants demonstrated some incorrect knowledge or lack of certainty regarding whether HIV can be transmitted through saliva but, on the whole, most were well-informed with respect to HIV transmission and prevention. Nonetheless, high levels of perceived contagiousness were reported. This suggests discordance between knowledge (e.g. HIV cannot be transmitted through casual contact) and behavior (e.g. avoidance or increased physical distance from PLWH), and is exemplified by comments in which participants indicated that, despite knowing that they cannot acquire HIV through casual contact, they would nevertheless be "careful" when in the presence of PLWH, "just in case".

If I know that someone is infected with HIV, then I think that I would be a little more distant with that person, and that is just automatic because we know that the disease is only transmitted through blood and fluids. So I think I would be a little more cautious. Somehow, there is fear in your body, I think. In my opinion, you would kind of put the handbrake on. (HIV-negative Surinamese man)

In our comparison of the communities, some differences between the communities were noted. In Antillean and Surinamese communities, the fear of contagion was predominantly 
related to acquiring HIV through physical contact while, in African communities, this fear also pertained to the air, perhaps because HIV and tuberculosis co-infection is prevalent in African countries. One participant stated, "Some people are just scared of even sitting close to me like you are sitting right now because they have no idea. They think it could come from the air and infect you" (HIV-positive Nigerian man). Another said, "People don't want to come near you. They don't know it is not contagious. They think it's like tuberculosis. They think it's in the air but it is not in the air" (HIV-negative Ghanaian man).

\section{Perceived severity}

High levels of perceived severity in African, Antillean, and Surinamese diaspora communities were also noted. HIV was considered by many HIV-negative participants to be a "death sentence" (HIV-negative Antillean man) and a "killer disease" (HIV-negative Congolese woman). This belief that HIV is analogous to death was vividly described by one participant as follows.

You know that you can't get rid of it and that you'll die. You'll have it for the rest of your life and you will need to take medicine to slow it so that it doesn't go really fast but you know that the end is in sight. ... However you look at it, the end is in sight. ... Your future's in the gutter, so to speak. ... It's not a fever for which you just take a couple of pills and in three days, you're better. You have to change your whole life, your lifestyle. You have to take medicine day and night to make it just a little easier otherwise you'll just rot away. It is horrible. (HIV-negative Surinamese man)

Evidently, the lifelong obligation to take medication was also seen by the above participant and others as a factor that contributes to the perceived severity of HIV: "You will be on a whole lot of medication for years. You live only to take those pills" (HIVnegative Antillean woman).

HIV-positive participants indicated that they too had associated HIV with death prior to and shortly after their diagnosis, as did their families and friends. One participant said that when he was told that he had HIV, he thought he had "come to the end of the road" (HIV-positive Nigerian man). Another said, "I thought, 'I'm going to go home, clean my whole house and prepare myself for death"' (HIV-positive Antillean woman). Yet another participant spoke of how her family looked at her as if she were a "time bomb" that could "drop dead" at any moment (HIV-positive Rwandan woman). Fortunately, many PLWH conveyed that they themselves and most of the people close to them have since come to realize that HIV is not a death sentence and that it is possible to live a healthy life with HIV. However, among those who are not close to PLWH, the idea that HIV is a fatal disease appears to persist, 
despite the acknowledgement of antiretroviral medication by approximately half of the HIV-negative participants.

Another related belief reinforcing the belief that HIV is very severe is that PLWH are "skinny". Participants from all three ethnic groups indicated that if someone loses weight and becomes thin, people will assume that that person has HIV: "Even my own junior brother, he used to have one of my friends and then tell me, 'That man is so pale, so slender, I suspect he has HIV'" (HIV-positive Cameroonian woman). Another HIV-positive participant exemplified this notion that weight and HIV status are connected when she told the interviewer about how her mother in Suriname was concerned about her being ill and was comforted over the telephone by the following words: "Mama, if you could see me, you'd see that I am 100 kilograms" (HIV-positive Surinamese woman). Clearly, this notion that HIV can be seen on a person is present in African, Antillean, and Surinamese communities, and is linked to beliefs regarding the course and severity of the condition. In essence, it exemplifies beliefs that PLWH are so ill that they wither away and die: "It just eats you all through and, at the end of the day, it just leaves you an empty frame - nothing else" (HIV-negative Kenyan woman).

The degree to which HIV was perceived as a very severe condition did not appear to vary between the ethnic communities. In all three ethnic groups, the majority of participants contended that HIV is a very serious disease. What was observed was that, in general, the HIV-negative participants tended to sketch a much bleaker and severe picture of life as a person with HIV than PLWH did.

\section{Personal responsibility}

Numerous HIV-negative participants indicated that PLWH are often thought to have acquired HIV through irresponsible, preventable action such as unprotected sex. As such, PLWH are seen as blameworthy for their infection: "They brought it on themselves" (HIV-negative Kenyan man). One Antillean participant contended that, "Getting HIV is not an accident that just happens to you. It's not like 'Oh poor you, you got HIV.' No, it is simply your own responsibility" (HIV-negative Antillean woman). Another claimed that "Surinamese people tend to think: it's your own fault and, in essence, that's true" (HIVnegative Surinamese man).

A few HIV-positive participants indicated that they indeed felt responsible for their HIV infection and see, or have seen, their infection as punishment for bad behavior: "I first thought that this was punishment - punishment from God, you know. I deserved it because of, you know, the lifestyle I had" (HIV-positive Surinamese man). 
In fact, especially among Surinamese and African participants, HIV is often seen as punishment from ancestors or God for poor or immoral behavior.

The way that people look at $[\mathrm{PLWH}]$ is like you are really immoral. That is why you get the disease. ... The first thing that comes to people's mind is that you must have been immoral and that is why you have it. (HIV-negative Kenyan woman)

You often hear: "It's a curse. You were bad." People think that it has something to do with the spiritual world and those kinds of things ... Winti things and then they say, "Your ancestors are angry with you and have cursed you." (HIV-negative Surinamese man)

The data further suggest that, because PLWH are held personally responsible for their infection, they are often met with less compassion and empathy than people who suffer from a condition other than HIV: "Why is that if someone loses a leg, people understand but if someone has HIV, they don't?" (HIV-positive Surinamese woman).

If someone gets cancer, then he gets compassion. If someone gets HIV, he doesn't get that easily. It's more a fear reaction and then, "Oh shit, you're going to die." ... I think that only hemophilia patients that have gotten HIV through a blood transfusion get the same kind of compassion or understanding. (HIV-positive Antillean man)

With respect to differences between the ethnic communities, we found that the role of personal responsibility as a contributing factor to stigmatization was less present, but surely not absent, in Antillean communities compared to Surinamese and African communities.

\section{Associations with norm-violating behavior}

Since the beginning of the epidemic, HIV has been associated with behaviors that are considered norm-violating or socially unacceptable. Our study found HIV to be associated with promiscuity, commercial sex work, and homosexuality.

\section{Promiscuity and commercial sex work}

Participants from all three ethnic communities reported that most people in their community associate HIV with being "loose" or "easy".

People who carry the disease: To us, they are loose - people who just do whatever and go to bed with whoever and aren't careful about things like using a condom or something. They just dive into bed with anyone and that is looked down upon. (HIV-negative Antillean woman) 
Participants also reported that this association between HIV and promiscuity most often pertains to women. Women "tend to be blamed to be the promiscuous; it falls more on the women" (HIV-negative Kenyan woman). The following excerpt from an interview illustrates this well.

If a girl has lots of boyfriends and those kinds of things, then she automatically has AIDS ... only because she is sexually active. They don't consider that maybe she just likes it and she does it safely. No, she is automatically a whore and she automatically has AIDS. For boys, that's [promiscuity] cool and manly. (HIV-negative Antillean woman)

In some cases, participants referred to commercial sex work as a specific form of promiscuity. This association was particular apparent among African participants: "Sometimes they think, 'Oh, she was a prostitute.' ... They will treat [her] like she is a hooker" (HIV-negative Kenyan woman). This was confirmed by African PLWH: "People think when you are HIVpositive - they label you as prostitute" (HIV-positive Rwandan woman).

When the association between promiscuity and HIV pertained to men, infidelity was frequently mentioned. In fact, among the Antillean participants, comments on infidelity pertained exclusively to men and, interestingly, were made exclusively by women, thus reflecting the structure of romantic relationships in Antillean communities where infidelity on the part of men is considered normal, but not appreciated, by women.

Logically, when participants spoke of the association between HIV and promiscuity, they also often mentioned an attribution of blame. One participant said, "There are still people who believe that they asked for it because they had many men or many women, because they are loose" (HIV-negative South African woman). Another participant described how people think about PLWH as follows.

[People say] "He's been/she's been sleeping around. You see? This is what you get!" It is not like it can happen by mistake. They are like "You looked for it." ... She has brought this disease to herself. She was promiscuous herself. She is the one who brought the disease. (HIV-negative Kenyan woman)

Unsurprisingly, HIV-positive participants were keenly aware that HIV is associated with promiscuity, commercial sex work, and infidelity, and that these behaviors also yield blame. This was exemplified by the fact that a number of HIV-positive participants explicitly, but without solicitation, defended their fidelity and emphasized that they did not acquire HIV through irresponsible behavior: "I didn't go around screwing everyone and anyone" (HIVpositive Surinamese woman). 
They see you as a whore and because you are a whore, you got the disease, and because of that, they don't want anything to do with you because you are a whore. "You sleep with whoever whenever" - that's how they think but it's not true. It's not true but they think that. (HIV-positive Antillean woman)

With respect to differences between the ethnic communities, we observed that the association between HIV and promiscuity was present in all ethnic groups but more common in African communities than in Antillean and Surinamese communities.

\section{Homosexuality}

The belief that HIV is a "gay disease" was very present and explicit among Antillean and Surinamese participants but not among African participants. Many HIV-negative Surinamese and Antillean participants made some reference to homosexuality when asked what beliefs are linked to HIV: "I think of gays that have the virus. Most people in my culture say that it is the gays that have it" (HIV-negative Surinamese woman). Another participant said, "Usually, gay men get it. That's kind of the idea - that only gay men have HIV" (HIV-negative Antillean woman). This belief was confirmed by PLWH: "Either way, people think it is a gay disease" (HIV-positive Antillean man).

Once again, attributions of personal responsibility were observed. Men who had sex with men were considered blameworthy for their HIV infection.

They say, well, that is more a gay thing - man and man - and that is not allowed and that is not good. You have some families that accept it but not many. From the time you are a kid, you know it is not good. Many Surinamese people think that it is not from God and that is why you get the disease. (HIV-positive Surinamese man)

\section{Cultural taboos}

Many of the participants from African, Antillean, and Surinamese communities contended that the beliefs that reinforce stigmatization of PLWH (i.e. HIV is highly contagious, very severe, acquired through volitional behavior for which one can be held personally responsible, and associated with promiscuity, commercial sex work, and homosexuality) are exacerbated and perpetuated by silence, denial, and taboo within their communities. One participant said, "In my community, some people don't want to talk about HIV. ... I know, [in] my community, when I start to talk about HIV, nobody want[s] to talk about this" (HIV-negative Guinean man). Another participant stated, "People often think that you can get HIV just by touching. They think that because they don't ever talk about it [HIV]" (HIVpositive Antillean man). 
The cultural taboo on talking about HIV in African, Antillean, and Surinamese communities also appears to be exacerbated by taboos on talking about all things related to sexuality in African, Antillean, and Surinamese culture.

You know what it is? The way in which you get infected is sexual. Of course you can get it through injection needles but [the Antillean community] assume[s] that it is always from sex and, among Antilleans, talking about sex is a taboo, and so you have this disease that you get through sex and the taboo only gets bigger. (HIV-negative Antillean woman)

When you think about HIV - in fact, I am talking about it right now because you are asking me those questions but it is a very silent disease. We actually don't even use the word HIV. It is coming more and more to light because of the fact that when you have this disease where we have given it various names because of the stigma surrounding the disease and the fact that it is not discussed. It is such a taboo to talk about HIVIAIDS and that is because it is a sexually transmitted disease. (HIV-negative Kenyan woman)

In Antillean and Surinamese communities, the taboo on talking about sexuality is even greater when it pertains to homosexuality.

I was thinking, "Why is [HIV] so stigmatized?" It has to do with the whole homosexuality thing that, on its own, is a stigma and a taboo. In some cultures, it's starting to come out a bit but it's not how it should be ... .so [stigma] is the result of that. So you are gay and you are also HIVpositive - you definitely can't talk about that. (HIV-positive Antillean and Surinamese man)

In short, in African, Antillean, and Surinamese communities, myths and beliefs about HIV and PLWH that contribute to HIV-related stigma are upheld by cultural taboos on talking about HIV, sexuality, and, in Antillean and Surinamese communities, also homosexuality. On a positive note, most participants did claim that the taboo in their ethnic communities in the Netherlands was less than the taboo in their home countries. Nonetheless, cultural taboos were considered to reinforce beliefs that promote the stigmatization of PLWH.

\section{DISCUSSION}

This study is, to our knowledge, the first to comprehensively document the beliefs that contribute to HIV-related stigma in African and Afro-Caribbean diaspora communities in the developed world and the cultural context of these beliefs. The findings suggest that, on the whole, the beliefs that have been found to contribute to HIV-related stigma in communities 
indigenous to developed countries also are held by members of diaspora communities originally from developing countries.

The findings show that, in African, Dutch Antillean, and Surinamese diaspora communities, the belief that HIV is highly contagious and that casual social contact can pose a risk for HIV infection persists despite adequate knowledge regarding HIV transmission. This discrepancy has previously been found in African studies (Maman et al., 2009; Ogden \& Nyblade, 2005), and studies conducted in developed countries have also found that people have aversions to casual contact with PLWH despite apparent awareness of how HIV is actually transmitted (Pryor, Reeder, \& Landau, 1999; Rozin, Markwith, \& Nemeroff, 1992). Research has shown that people often manifest an automatic and immediate aversion to stigmatized persons that can be followed by a controlled and thoughtful reaction. The controlled and thoughtful reaction is contingent upon adequate knowledge about HIV transmission, time to consider one's reaction, and the motivation not to stigmatize (Pryor, Reeder, Yeadon, \& Hesson-McLnnis, 2004). Our study shows that when people are unsure about the fine details of HIV transmission, they tend to err on the side of caution and avoid $\mathrm{PLWH}$, thus suggesting that interventions aimed at the reduction of HIV-related stigma should seek to reduce this lack of certainty by providing information on how HIV is not transmitted in addition to building skills pertaining to social interactions with PLWH (Bos, Schaalma, \& Pryor, 2008; L. Brown, Macintyre, \& Trujillo, 2003; Ogden \& Nyblade, 2005).

The second belief contributing to HIV-related stigma in African and Afro-Caribbean communities is the belief that HIV is a very severe disease. Participants frequently associated HIV with death and wasting. This association is not surprising given that many members of diaspora communities originate from countries where highly active antiretroviral therapy (HAART) has, until recently, been or currently remains either unavailable or difficult to access. HIV-stigma reduction interventions should therefore aim to increase awareness of HAART in diaspora communities and convey, through information and personal contact with PLWH, that HIV is, at least in the developed world, a chronic condition and that PLWH can lead long and healthy lives with HIV (Bos, Schaalma, \& Pryor, 2008; Ogden \& Nyblade, 2005).

The third belief is the belief that PLWH are personally responsible for their HIV infection. In our study, we found that attributions of blame are common in African, Antillean, and Surinamese diaspora communities, and that HIV is frequently considered due justice for immoral or irresponsible behavior, thus yielding less compassion for PLWH than for people with other medical conditions. In some cases, the punishment is considered to come from God; in others, it comes from ancestors. Attributions of blame have previously been found 
in a quantitative study conducted by Visser and colleagues (2006) in South Africa. In their study, $61 \%$ of the participants considered PLWH to be of poor moral character, $22 \%$ considered HIV to be punishment for bad behavior, and 26\% blamed PLWH for contracting HIV. Interventions aiming to counter the effect of attributions of personal responsibility can focus on generating empathy for PLWH through, for example, repeated personal and/ or vicarious contact with PLWH (Bos, Schaalma, \& Pryor, 2008; L. Brown, Macintyre, \& Trujillo, 2003; Herek \& Capitanio, 1997). Such contact not only has the potential to increase awareness of HIV and decrease fear reactions to PLWH; it has also been established as an effective means of inducing sympathy and compassion for stigmatized individuals and subsequent stigma reduction (Bos, Schaalma, \& Pryor, 2008; R. Brown \& Hewstone, 2005; Norman, Abreu, Candelaria, \& Sala, 2009; Pettigrew \& Tropp, 2006; Visser, Makin, \& Lehobye, 2006).

The fourth belief contributing to HIV-related stigma is the belief that PLWH have acquired HIV because they engaged in apparently norm-violating behavior. Our findings suggest that these associations can differ from one culture to another. For example, in our study, HIV was associated with homosexuality only in Afro-Caribbean communities and not in African communities. In African communities, the association with commercial sex work appeared stronger than in Afro-Caribbean communities. In all three ethnic groups, HIV was associated with promiscuity but, in Antillean communities, promiscuity was, according to female participants, analogous to male infidelity. Also, unlike the developed world, in our study, HIV was not associated with intravenous drug use. This suggests that the associations made are indeed impacted by culture and the nature of the epidemic in the regions from which diaspora originate. Changing negative attitudes toward promiscuity, commercial sex work, and homosexuality is difficult as such attitudes are often deeply entrenched in one's culture. HIV-related stigma reduction interventions can seek to provide stereotype-inconsistent information about HIV and PLWH, and offer a safe environment in which community members can discuss their stigma-related values and beliefs (Bos, Schaalma, \& Pryor, 2008; Nyblade, Pande, Mathur, MacQuarrie, \& Kidd, 2003; Ogden \& Nyblade, 2005) but more is needed. Societal and community structures as well as cultural beliefs that judge promiscuous behavior, reinforce negativity toward commercial sex work, and oppress men who have sex with men must be addressed (Kalichman \& Simbayi, 2003; Parker \& Aggleton, 2003). This requires public commitment from all sectors of government as well as community leaders and the media (Visser, Makin, \& Lehobye, 2006).

An additional finding of our study is that cultural taboos on talking about HIV and sexuality in African, Antillean, and Surinamese communities exacerbates the abovementioned beliefs. In Afro-Caribbean communities, the taboo on sexuality extends also to homosexuality. 
The contributing role of taboo to HIV-related stigma has previously been noted by studies conducted in Sub-Saharan Africa (Campbell, Foulis, Maimane, \& Sibiya, 2005; Roura et al., 2008) and in studies with African and Caribbean diaspora (Burns, Imrie, Nazroo, Johnson, \& Fenton, 2007; Dodds, 2006; Gardezi et al., 2008). Taboos can perpetuate myths regarding HIV transmission and severity while reinforcing attributions of blame and norm-violation. Breaking cultural taboos on HIV and sexuality is thus imperative if stigmatization toward PLWH is to be reduced. Public disclosure to one's community on the part PLWH can break taboos and thereby contribute to stigma reduction but must be applied cautiously as such disclosure can have both positive and negative consequences (Paxton, 2002b; Stutterheim et al., 2009). It is therefore important that PLWH who want to contribute to breaking taboos and reducing stigma be empowered and adequately equipped to deal with negative reactions. Interventions that support PLWH and train them in how to best disclose their status and how to cope with potential negative reactions must precede interventions that utilize PLWH as public spokesmen to break taboos and reduce stigma (Bos, Schaalma, \& Pryor, 2008; L. Brown, Macintyre, \& Trujillo, 2003).

In their totality, our findings point to the need for community-based interventions in African, Antillean, and Surinamese diaspora communities that are geared to changing beliefs regarding contagiousness, severity, and personal responsibility, and reducing associations between HIV and norm-violating behavior such as promiscuity, commercial sex work, and, in Afro-Caribbean communities, also homosexuality. Interventions should also seek to break cultural taboos on HIV and sexuality. In order to be effective, these communitybased interventions must be rooted in theory and evidence, and involve both HIV-negative and HIV-positive community members throughout all phases of intervention development, implementation, and evaluation (Bos, Schaalma, \& Pryor, 2008; L. Brown, Macintyre, \& Trujillo, 2003). In the absence of such interventions, HIV-related stigma is likely to persist and have negative consequences for not only PLWH but also their communities. 


\section{HIV-RELATED STIGMA IN AFRICAN AND AFRO-CARIBBEAN COMMUNITIES IN THE NETHERLANDS: MANIFESTATIONS, CONSEQUENCES, AND COPING}

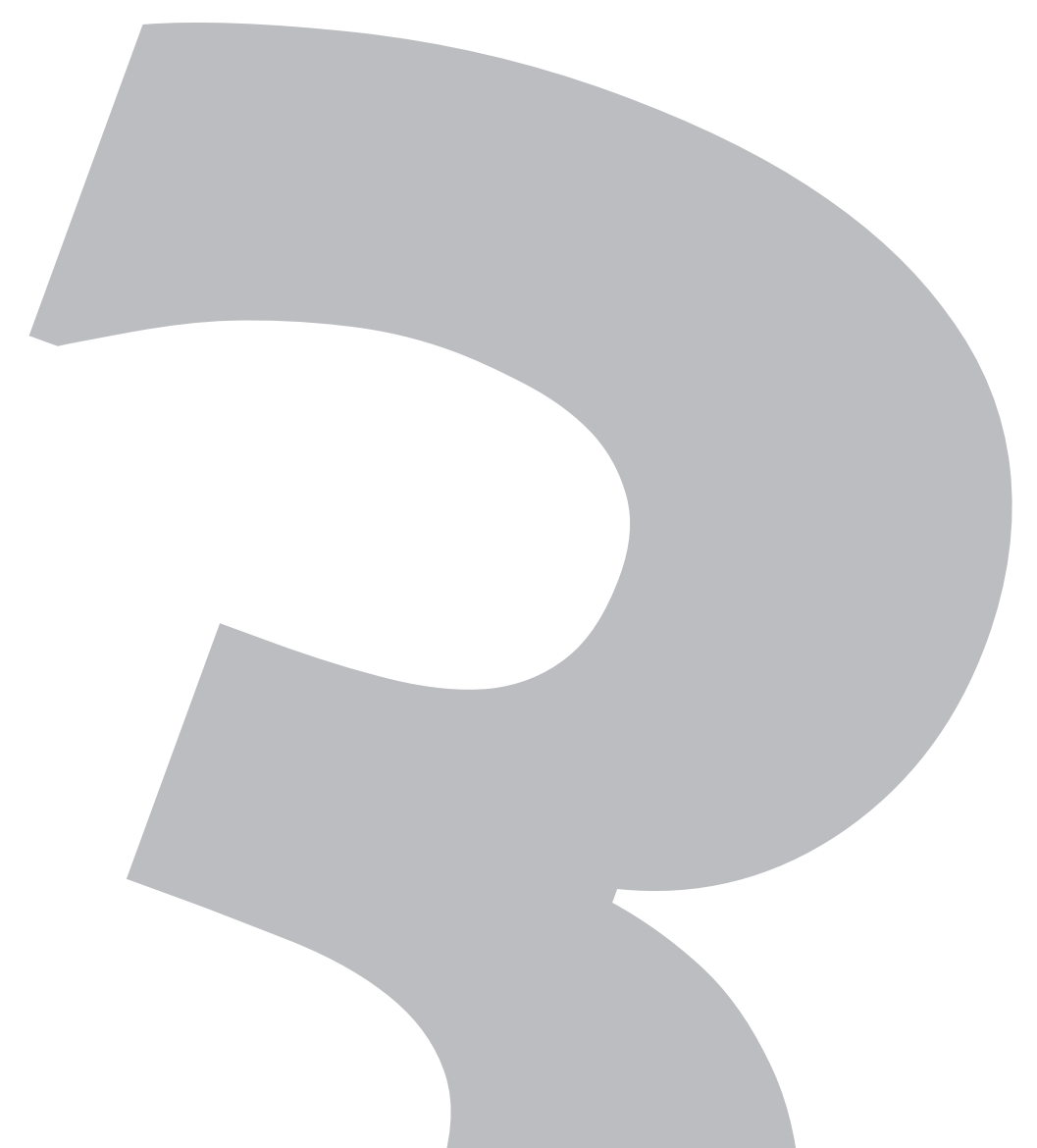

This chapter has been submitted for publication in similar form as:

Stutterheim, S. E., Bos, A. E. R., Shiripinda, I., de Bruin, M., Pryor, J. P., \& Schaalma, H. P. HIV-related stigma in African and Afro-Caribbean communities in the Netherlands: 


\section{ABSTRACT}

HIV-related stigma in African and Afro-Caribbean diaspora communities in the Netherlands was investigated. Interviews with HIV-positive and HIV-negative community members demonstrated that HIV-related stigma manifests as social distance, physical distance, words, and silence. The psychological consequences of HIV-related stigma reported were emotional pain, sadness, loneliness, anger, frustration, and internalized stigma. The social consequences included decreased social network size, limited social support, and social isolation, and resulted from not only enacted stigma but also self-imposed social withdrawal. Also, poor treatment adherence was a health-related consequence. People living with HIV (PLWH) employed both problem-focused and emotion-focused coping strategies to mitigate the negative consequences of stigma. Problem-focused coping strategies included selective disclosure, disengagement, affiliating with similar others, seeking social support, and, to a lesser extent, activism. Emotion-focused strategies included distraction, positive reappraisal, religious coping, external attributions, disidentification, and acceptance. HIV-related stigma clearly permeates African and Afro-Caribbean communities in the Netherlands, and should be targeted for intervention. 


\section{INTRODUCTION}

HIV is not merely a condition that impacts the physical health of those infected. It is a condition that has major social and psychological implications that are rooted in perceptions about what it means to have HIV. HIV is, in fact, a highly stigmatized condition because it is often thought to be highly contagious, very severe, and the result of irresponsible volitional behavior considered by many to be norm-violating such as commercial sex work, homosexuality, and promiscuity (Bos, Dijker, \& Koomen, 2007; Dijker \& Koomen, 2003; Herek, 1999; Lichtenstein, Laska, \& Clair, 2002). Stigmatization is, in essence, a systematic and complex process of devaluation whereby a person is considered to possess a discrediting attribute and subsequently deemed tainted or flawed by others (Crocker, Major, \& Steele, 1998; Goffman, 1963; Jones et al., 1984; Parker \& Aggleton, 2003).

Stigmatization toward people living with HIV (PLWH) affects the treatment of PLWH in a number of ways and across a broad range of settings. Manifestations include avoidance, exclusion, rejection, social ostracism, blaming, violence, physical distance, indifference, and awkward social interaction. Relevant settings are families, communities, friends, sexual relationships, health care, housing, the financial services sector, religious institutions, work, and educational settings (Andrewin \& Chien, 2008; Greeff et al., 2008; Malcolm et al., 1998; Shamos, Hartwig, \& Zindela, 2009; Stutterheim et al., 2009; Varas-Diaz, SerranoGarcia, \& Toro-Alfonso, 2005). The consequences of HIV-related stigma are severe and include hampered HIV prevention efforts, testing delays, problematic treatment adherence, psychological distress, and disrupted social interactions (Bos, Schaalma, \& Pryor, 2008; Brooks, Etzel, Hinojos, Henry, \& Perez, 2005; Rintamaki, Davis, Skripkauskas, Bennett, \& Wolf, 2006; Stutterheim et al., 2009; Vermeer, Bos, Mbwambo, Kaaya, \& Schaalma, 2009). In an effort to mitigate the negative psychological and social impact of HIV-related stigma, PLWH can employ a number of coping strategies that are geared to either altering the relationship between the person and the environment (problem-focused coping) or to regulating negative emotions (emotion-focused coping). Problem-focused coping strategies can target the self, the situation, or others, and include selective disclosure, compensating for the stigma during social interactions, avoiding situations where stigmatization is likely (disengagement), affiliating oneself with similar others, seeking social support, and activism. Emotion-focused strategies documented include downward social comparison, external attributions, denial or prejudice minimization, distraction, positive reappraisal, and disidentification with the stigmatized identity (Crocker, Major, \& Steele, 1998; Galvan, Davis, Banks, \& Bing, 2008; Major \& O’Brien, 2005; Medley, Kennedy, Lunyolo, \& Sweat, 2009; Miller \& Kaiser, 2001). 
Since the early days of the epidemic, the prevalence of HIVIAIDS has been highest in Sub-Saharan Africa and the Caribbean. As a logical consequence, African or AfroCaribbean diaspora communities also have high prevalence rates for HIV. For example, in the Netherlands, non-Western migrants comprise one tenth of the Dutch population but one third of all HIV cases. Of that third, more than half originate from Sub-Saharan Africa and almost a third from the Caribbean (Shiripinda \& van Eerdewijk, 2008). Although these data clearly demonstrate the severity of the epidemic in these communities and previous research has shown that African and Afro-Caribbean PLWH experience extensive stigmatization (M. Anderson et al., 2009; Bond, Chase, \& Aggleton, 2002; Carr, 2004; Nyblade, Pande, Mathur, MacQuarrie, \& Kidd, 2003), there is a paucity of literature on HIV-related stigma in African and Afro-Caribbean diaspora communities. To our knowledge, the literature is limited to a few studies on African immigrants in the United Kingdom (Burns, Imrie, Nazroo, Johnson, \& Fenton, 2007; Dodds et al., 2004; Kinniburgh, Scott, Gottlieb, \& Power, 2001), one study on predominantly Sub-Saharan African PLWH in the Netherlands (Shiripinda \& van Eerdewijk, 2008), one study on Caribbean communities in the United Kingdom (M. Anderson et al., 2008), and one study on African and Caribbean communities in Canada (Gardezi et al., 2008). Although these studies have documented a fear of stigmatization among African and Afro-Caribbean diaspora PLWH and some of the manifestations and consequences of HIV-related stigma in their communities, comprehensive knowledge of the manifestations and consequences of HIV-related stigma in African and Afro-Caribbean diaspora communities is still lacking, as is information on how these PLWH cope with stigma. More research in terms of not only volume but also comprehensiveness and rigor is necessary to, first, support PLWH subjected to HIV-related stigmatization and, second, develop culturally appropriate community-based stigma reduction interventions. To help meet these needs, the current study endeavored to: 1) document manifestations of HIV-related stigma in African and Afro-Caribbean communities in the Netherlands from the perspectives of both PLWH and their fellow community members; 2) delineate the psychological, social, and health-related consequences of HIV-related stigma for these PLWH; and 3) explore the strategies these PLWH employ to cope with stigmatizing experiences.

\section{METHODS}

Following study approval by Maastricht University's Ethics Committee, members of African, Dutch Antillean, and Surinamese communities were recruited for face-to-face, semistructured interviews of approximately an hour by a researcher (SS, IS, MB) or by 
one of twelve peer interviewers employed and trained by the researchers. Recruitment was purposeful and occurred directly through interviewers or through announcements distributed by the Dutch HIV Association, Humanitas Foundation, or HIV nurses working in Dutch hospitals. Both HIV-positive and HIV-negative African, Antillean, and Surinamese community members were included in the study as this allowed for triangulation across data sources. Our sample comprised 42 HIV-positive participants, of which 16 were African, 9 Antillean, 16 Surinamese, and 1 both Antillean and Surinamese, and 52 HIV-negative participants, of which 16 were African, 19 Antillean, and 17 Surinamese. Once recruited, participants were given information regarding the study's purpose and procedure. Informed consent was obtained and a monetary reward of $€ 30$ was provided after interview completion. The interviews were held between January 2005 and May 2008 and were guided by a structured protocol of open-ended questions with follow-up probes. The protocol for HIV-positive participants explored how others have reacted to their HIV status, the consequences of negative reactions, and how one has dealt with negative reactions. The protocol for HIV-negative participants explored how PLWH are treated in African and Afro-Caribbean diaspora communities in the Netherlands.

To enhance rigor, all interviews were recorded with a digital voice recorder and transcribed verbatim. Data were processed with QSR NVivo 2.0 and analyzed using a general inductive approach. Each transcript was read thoroughly while listening to the corresponding recording to identify emerging themes and establish categories to which text fragments were assigned. As coding occurred, categories and subcategories were linked to one another. A decision trail was maintained. All emergent categories were documented, as were changes made to the categories and the rationale for those changes. Coding continued until saturation was evident. Findings were subsequently checked with relevant stakeholders in the African, Antillean, and Surinamese communities in the Netherlands.

\section{RESULTS}

\section{Manifestations of HIV-related stigma}

\section{Stigmatization through social distance}

PLWH extensively reported manifestations of HIV-related stigma that reflect an increase in social distance (e.g. avoidance, rejection, abandonment, and social exclusion). Many reported experiencing greater social distance in specific settings. One such setting was the family: "I used to go to [my family's] home. They would invite me, you know. I don't get invited anymore. It's like I've been forgotten" (HIV-positive Surinamese man). Another 
was with friends: "[l have] friends who did not want to cooperate with me because I was HIV-positive, who did not want to really see me anymore" (HIV-positive Kenyan man). Yet another very relevant and important setting for many PLWH was romantic partners. Some participants reported abandonment by the partner they were with at the time of diagnosis: "The relationship was good. When he heard I had HIVIAIDS, he stayed for a bit but, after a while, he was [snaps her fingers to imply 'gone']" (HIV-positive Antillean woman). Participants also reported rejection by new or potential partners: "When I told a guy that I am HIV-positive, he said, 'I'll call you,' but he never did" (HIV-positive Surinamese woman).

HIV-negative community members also acknowledged the avoidance, rejection, abandonment, and exclusion of PLWH. One Antillean woman said, "As soon as people know that someone has the virus, that person is no longer part of the group. They would rather not spend time with that kind of person." In fact, a number of community members indicated that PLWH are seen as "pariahs" or "untouchables": "That person essentially gets a label. For people who don't know him well, he is, in a matter of speaking, really a 'pariah'” (HIV-negative Antillean woman).

It is almost as though they are the "untouchables". There is this sympathy but a displaced sympathy. There is sympathy as long as they are at a distance from you. You know, you sympathize at a distance but it is not that you welcome them in your home and take care of them. There is sympathy for as long it is not your business. (HIV-negative Kenyan woman)

Increased social distance might result from perceptions that HIV is very severe. It might also occur because of moral judgments, as exemplified by the following citation from a Surinamese community member: "If you hear that someone has HIV, you always think, 'Distance!' because people associate HIV with bad behavior, and people think, 'He's going to die anyway. He's going to die and I want nothing to do with him." (HIV-negative Surinamese man).

\section{Stigmatization through physical distance}

In addition to experiencing social distance, PLWH, and particularly African PLWH, reported increased physical distance in social interactions. One participant said, "If he [a friend] greets me, if we were kissing before, now he would give the hand from one meter or two meters" (HIV-positive Burundian woman). HIV-negative participants confirmed that people do maintain increased physical distance from PLWH. Some reported that if they were to encounter a PLWH, they would indeed maintain physical distance: "I'd prefer to have as little contact - physical contact with that person as possible" (HIV-negative Antillean 
woman). PLWH furthermore reported that some people not only maintain physical distance but also avoid actual physical contact with them. Numerous PLWH reported incidents whereby people were unwilling to sit next to them, touch them, or shake their hand.

Participant: There are some people whom you tell and they do not have much knowledge and therefore they still think they get AIDS maybe by handshaking or maybe by -

Interviewer: So don't they shake your hand? They shake hands with the other ones?

Participant: Yeah, they "Hi". You know, that "Hi" and waving at you. They just wave and say, "Hi, how are you?", and then that's it. That is how it ends. Interviewer: Were they hugging you before?

Participant: Yeah, before we had like really a very good relationship, not hugging as such, but we would shake hands.

(HIV-positive Zimbabwean woman)

PLWH reported that increased physical distance even occurs within romantic relationships: "[My ex-husband] didn't want to eat or drink with me. Sometimes, he didn't want to sleep next to me ... He found it difficult to touch me. He wore gloves" (HIV-negative African woman). This fear of physical contact appears to extend also to objects PLWH touch such as food, dishes, toilets, and chairs. One participant said, "[My relatives] don't feel very comfortable for me to handle food, especially food that you eat without cooking like apples or whatever" (HIV-positive Zimbabwean woman). Another told the interviewer, "[I heard my brother] saying to the wife, 'Whatever he uses, keep it somewhere different. Don't mix it: the cups, the glasses, the spoons'" (HIV-positive Kenyan man). HIV-negative community members confirmed this. One participant said, "I wouldn't drink out of the same glass" (HIV-negative Surinamese woman). Another stated, "If I see a person with AIDS and that person has sat on a chair, if he gets up, I won't go sit on that chair" (HIVnegative Antillean man).

\section{Stigmatization through words}

Participants frequently reported stigmatizing words. HIV-positive and HIV-negative participants indicated that particularly gossip but also blaming, negative remarks, and disdain occur within their communities. Gossip was the most frequently cited manifestation among Antillean and Surinamese participants. Many PLWH described having been the subject of gossip. One participant said, "I told some friends, my closest friends actually, and later people came up to me and said, 'So and so told me that you have 'it'" (HIVpositive Antillean man). Another said, "I chose to tell my cousin and another aunt, and I know for sure that they didn't keep it to themselves. They told other people" (HIV-positive 
Antillean woman). Ex-partners were also a source of gossip: "I went to the doctor with my boyfriend at the time and he told all his friends. I wasn't happy about that because I think it is something private - my private matter" (HIV-positive Antillean man).

In similar vein, a number of African PLWH reported violations of confidentiality by religious leaders, community and social organizations, and the health care sector. One participant indicated that her pastor failed to keep her secret: "He is telling people he is trying to break [stigma and taboo], that people don't have to be laughing at [PLWH] but he is the one who is not keeping secrets for people having HIV" (HIV-positive Zambian woman). Another participant said that a member of an organization that had his status on file approached him and asked if he was the PLWH on file: "I thought my information was confidential. My information is not very confidential" (HIV-positive Ugandan man). Yet another participant spoke of a social worker who had informed others of her status: "She went and told someone else and, one day, they came to my caravan to see what an HIV-positive person looks like" (HIV-positive Burundian woman). Other participants claimed that health professionals had violated their confidentiality: "After the test, the doctor invited [my cousin] and even told him about the test before telling me. ... The doctor let the news out to my cousin before I saw him" (HIV-positive Nigerian man).

HIV-negative participants confirmed the prominent role of gossip: "Rumors will spread and, before you know it, the whole community will know" (HIV-negative Surinamese man). Many Antillean and Surinamese participants claimed that gossip and rumors are, in fact, part of their culture because "everyone knows everyone. We all know each other and we have, in this small community, practically grown up together as brothers and sisters" (HIVnegative Surinamese man). Participants also reported that the Surinamese and Antillean communities use online forums to spread gossip: "There is a site and if someone knows that someone else has AIDS, they can put it on the site with a picture" (HIV-negative Antillean woman).

In these communities, gossip often occurs under the guise of a warning. People frequently inform others about community members' status so they can, in turn, avoid potential infection. One participant said, "If I was just standing there having a conversation with a guy, they go to him after the fact and say, 'You need to be careful because that girl has AIDS" (HIV-positive Surinamese woman). Another PLWH who was discrete about his status dated a woman who was less discrete and was warned by others that he should be careful: "They came to me and said, 'Do you know that this girl has AIDS - that this girl is sick and you must check yourself?'” (HIV-positive Nigerian man). 
Participants also reported stigmatization in the form of blaming, negative remarks, and disdain, although less frequently than gossip. One participant said that others have commented that she "has slept with all sorts of men and that is why I'm now sick" (HIVpositive Surinamese woman). Another indicated that her husband and his relatives blamed her for apparently bringing HIV home: "They were saying that I contaminated him" (HIV-positive Burundian woman). Yet another participant reported blaming by a health professional: "I went to have my uterus examined and I was admitted. The gynecologist was a woman and she, of course, had read my status and she was really rude ... [she said] stuff like 'You shouldn't have gotten that'” (HIV-positive Surinamese woman).

Other participants, who had chosen to conceal their status, reported having heard others make comments about PLWH that reflect blaming or are negative: "I have talked to people, intelligent people, who say, 'Cancer is something that happens to you but HIV is something you've done yourself. You're in essence responsible for it happening'" (HIVpositive Antillean man).

A number of PLWH in our study also claimed that, even when people do not explicitly blame them or subject them to negative remarks, they nonetheless look down on them and treat them with disdain. One participant said, "Nobody will dare respect you or talk nicely to you if you are infected" (HIV-positive Cameroonian woman). HIV-negative community members acknowledged this. One participant claimed that, "in no time, people no longer look up to you but rather down at you" (HIV-negative Surinamese man). Another said that PLWH are "second rate citizens" (HIV-negative Antillean woman).

\section{Stigmatization through silence}

According to the participants, both HIV-positive and HIV-negative, HIV-related stigma manifests not only through distance and words but also through silence. Numerous PLWH reported that particularly their family never mention or discuss their HIV infection. For these people, it is as if they never disclosed their status: "They know but they act like they don't" (HIV-positive Surinamese woman). Another participant said, "Some of them just ignore it if I tell them. They don't ask again ever. They just pretend as if I didn't say anything" (HIVpositive South African woman). Yet another conveyed, "It's never talked about. They don't ask about it. They don't say anything" (HIV-positive Antillean man).

In some cases, silence and denial ensues even after PLWH communicate a desire to talk about HIV: "When I want to talk about it, especially with family, they don't want to hear about it" (HIV-positive Antillean woman). Another said that, when she talks to her family about HIV, 'their reaction is 'What? What are you talking about? No.' It's total denial. It's 
like there is nothing going on. They never talk about. They never ask and when I bring it up, they change the subject" (HIV-positive Surinamese woman).

Silence and denial within families might be rooted in familial shame or fear of being stigmatized by association (Neuberg, Smith, Hoffman, \& Russell, 1994). It might also reflect taboos surrounding HIV in African, Antillean, and Surinamese communities. Some participants claimed that their communities in the Netherlands and back home do not want to acknowledge that members of their community indeed have HIV.

Participant: My mother recently said in passing that [a family member] had AIDS, and then she moved on to talking about something else, and that is typical for Surinamese people.

Interviewer: That people would rather not say it?

Participant: Exactly! You know, like, most people that die of HIVIAIDS had 'cancer'. You know, those kinds of things are said.

(HIV-positive Surinamese woman)

\section{Consequences of HIV-related stigma}

\section{Psychological consequences}

With respect to the psychological impact of stigmatization, PLWH conveyed that stigmatizing reactions and particularly those related to social distance (avoidance, rejection, abandonment, and social exclusion) had brought them emotional pain, sadness, and loneliness. One participant said, "It hurts like hell. You just want to crawl in a hole and stay there forever" (HIV-positive Rwandan woman). Another said, "You really feel lost. You really feel like you have been thrown away; you are being neglected now because of your status" (HIV-positive Zimbabwean woman). Yet another said, "At that moment, when those things happen, you feel really small" (HIV-positive Antillean man). PLWH reported that rejection from a (potential) romantic partner and exclusion from dating is particularly painful: “Every time you hear that, 'Nah,' or 'I'll call you', it's painful. It's a disappointment' (HIV-positive Antillean woman).

In addition to pain, sadness, and loneliness, a number of PLWH conveyed anger and frustration. One participant said that a stigmatizing experience "made me feel different from other people and it made me feel very uncomfortable. In fact, it annoyed me. It made me very angry" (HIV-positive South African woman). Another said, "It makes me depressed and frustrated because I think that at this point in time, people with HIV should be accepted" (HIV-positive Surinamese woman). 
A final psychological consequence of stigmatization observed was the internalization of stigma by some PLWH. One participant indicated that she no longer kisses her nieces and nephews because she wants to protect them from infection: "There is, in you, this feeling that says, 'I shouldn't be doing that. I shouldn't be kissing'” (HIV-positive Zimbabwean woman). Another two male participants said they would never have sex with a woman again: "I have never told a girl that I have AIDS. It's been four years since I've made love. I don't do it because I am scared to infect someone and I don't want to do that to someone" (HIV-positive Surinamese man). This same participant indicated that his HIV infection "is punishment from God" (HIV-positive Surinamese man) thus reflecting the internalization of HIV-related stigma.

\section{Social consequences}

The negative social implications of HIV-related stigma include a reduced social network, lack of social support, and social isolation. These are the result of not only enacted social exclusion but also self-imposed social isolation. Many PLWH reported having voluntarily withdrawn from their social circles in an effort to avoid situations in which they would be compelled to disclose their status and/or be subjected to stigmatization. One participant said, "I don't call because I don't know how to tell them that I am HIV-positive and I don't know how they will react" (HIV-positive Surinamese man). Another said that he avoids social situations because "imagine that the question arises, that you have a conversation and things come up, and you think, 'I have to tell them"' (HIV-positive Antillean man). Participants also conveyed that they not only find it difficult to maintain their old social circles, they also find it hard to meet and spend time with new people.

When I meet new friends, I don't feel like I will tell them about myself because if I like people, I don't want to lose people like I lost the other friends, and that is very difficult for me. It is living with your friends without telling them who you are, what you are, and what about your health and all these kinds of things. It is not easy. It makes life kind of difficult. (HIVpositive Kenyan man)

Again, participants emphasized the impact of stigmatization on dating and romantic relationships. A number of PLWH conveyed the difficulties they experienced when starting a new relationship.

Whenever you want to start a relationship, that's when it comes to mind: stigma. ... Right now, I don't have a relationship but I have met a guy, and, for me, it's really hard to talk about it. I sometimes throw things into the conversation, some topics, so I can get an idea of what he thinks, and then I think, "Wow, if I tell him, that'll be the end of it." ... I asked him whether 
he would have a relationship with someone who has HIV and he told me, "I wouldn't do that. It would be a hindrance in my life, for my sex life," and, from that, you know how someone thinks about it. ... If you want to start a romantic relationship, you are going to have to tell that person at some point or another, and that person will choose to do it or not to do it but nine out of ten times, they won't do it. (HIV-positive Antillean and Surinamese man)

Some participants indicated that their fear of rejection from a potential partner is such that they no longer bother with new relationships because they believe that disclosure to a new sexual partner will inevitably lead to stigmatization.

Anytime you want a relationship with someone, you have to think, "I am sick. I have HIV and how am I going to tell this person?" After a while, you reach a point that you don't want to do it. ... I am alone and I don't have a partner, and I don't want a partner because you'll tell that person and maybe he will push you away because you have HIV. No, l'd rather be alone with my kids. (HIV-positive Antillean woman)

\section{Health consequences}

HIV-related stigma also affects health both indirectly through its influence on psychological well-being and social support, and directly by complicating HIV treatment adherence. Quite a few PLWH in this study outlined situations in which fear of stigmatization has prevented them from taking their antiretroviral medication on time. One participant said, "If I am at a party and I need to get up and take my medication, people will ask why I do that so I just don't do it" (HIV-positive Surinamese woman). Another stated, "One of my sisters is a nurse. If she sees my pills, she'll know so I don't take my pills if I'm with her" (HIV-positive Surinamese man). Yet another illustrated, in detail, the difficulties HIV-related stigma poses for treatment adherence.

During the summer vacation, I often went to amusement parks and then you are in the bus and you agree to meet back at the bus at 17:00 so you can go home and then it is 18:00 and I am in the bus. I am sitting there with all sorts of people around me. There is someone sitting next to me who doesn't know and someone in front of me and she doesn't know it either, and she keeps turning around to talk, and there was no toilet in the bus, and, even if there was, it would be weird because, like, who would go to the toilet in the bus with a cup? So it was weird and I needed to take them so I was thinking, "Oh God, how am I supposed to do this? How am I supposed to do this?" (HIV-positive Surinamese woman)

Clearly, for some PLWH, keeping one's HIV status a secret takes priority over taking one's medication on time. 


\section{Coping with HIV-related stigma}

In an effort to mitigate some or all of the negative psychological, social, and healthrelated consequences of stigmatization, PLWH employ, often simultaneously, a number of coping strategies.

\section{Problem-focused coping}

Problem-focused strategies can target the self, the situation, or others (Miller \& Major, 2000). A self-focused strategy frequently cited was concealment or selective disclosure. A number of PLWH indicated that they are very selective in their disclosure because it reduces the likelihood of stigmatization: "I don't want my problem known to people. I try as much as I can to keep it secret" (HIV-positive Nigerian man).

A frequently reported situation-focused coping strategy was disengagement or social withdrawal. As mentioned early, many PLWH in this study reported intentionally avoiding situations in which stigmatization is likely. One participant said, "One of my brothers is very disappointing to me because he won't let me be around his children so I decided not to visit him anymore" (HIV-positive Kenyan man). Another participant conveyed the following:

My own aunt said to my sister, "If your sister comes here, she needs to sit off to the side, and we'll give her her own glass and spoon." I heard that and, of course, you feel down because your own family thinks that. Then I said to my sister, "Ok, I don't need to go visit her anymore." (HIV-positive Surinamese woman)

Avoidance of stigmatizing situations and people who stigmatize often parallels increased identification with people who share the same stigma, as exemplified by the excerpt below.

You realize that society is now taking a negative attitude toward you so you tend to look for those people who are in the same situation like you because these are the people who understand. You share the same experiences. Maybe they are also facing the same things that you are facing from society so you tend to click now with those people and when you come together, when you discuss these things, you kind of develop coping mechanisms to deal with the situation. You help each other when these things happen. [You ask,] "How have you done it?" and someone gives you an advice: "It happened to me and I did it this way." So, it is after society is showing a negative attitude towards you, and then you lean on those people who are in the same boat. (HIV-positive Zimbabwean woman)

PLWH not only sought support from other PLWH but also from nonjudgmental friends and family. In fact, many participants said that social support from their immediate environment 
helps them to cope with stigmatizing experiences: "As long as the people in my immediate surroundings, like my kids, are good about it, I don't really care what anybody else thinks" (HIV-positive Surinamese woman). Another example is as follows:

If the people close to you accept you as you are and support you in who you are - it's because of that that I feel strong, that I can take on the rest of the world. I don't care how the rest of the world thinks of me and how they'll react to [my HIV infection]. (HIV-positive Surinamese man)

A final problem-focused coping strategy geared to others is activism. Among the PLWH in our study, collective action and efforts to educate others occurred infrequently perhaps because of the cultural taboo surrounding HIV in these communities. Only two participants in our study (both Surinamese) engaged in some form of activism to change perceptions about HIV and PLWH.

\section{Emotion-focused coping}

In addition to problem-focused strategies, PLWH reported a number of emotion-focused coping strategies. One was distraction or focusing on things or people other than stigmatizing experiences. One participant said that stigmatization "makes you sad but I have two children and I just keep going. I think if I were alone that it would be much harder but l've got these two kids. They are my comfort and they keep me busy" (HIV-positive Surinamese woman).

Another emotion-focused coping strategy is positive reappraisal. A number of PLWH sought positive meaning in stigmatizing experiences, as exemplified by the citation below.

I go out a lot with friends. I see my family - visit my family. I can say that [HIV] makes you realize that life is too short and, also, I help others. Before it was like "me and my family", "me and my kids" but now, where I can help others, I go out of my way and help them. (HIV-positive Zimbabwean woman)

A number of participants also indicated that they cope with stigma by seeking comfort in their faith.

You see, the Bible says that my life is in his hands. You understand? The word of God offers me a lot when it comes to dealing with these things. Like I said, if I didn't have the Lord, I wouldn't know how to deal with all this HIV misery and the way people talk about it. (HIV-positive Surinamese man)

Other PLWH reported engaging in external attributions. They claimed that stigmatization is the result of ignorance on the part of others. 
When I think about [a stigmatizing experience], I still feel angry but, then again, I feel for those people because I know now that they are doing no better. They did what they thought was protecting themselves. It was a lack of information and education. (HIV-positive Rwandan woman)

Still others coped by distancing themselves from their stigmatized identity (disidentification). Many participants made comments suggesting that they would rather not base their personal identity and self-worth on their HIV infection.

Up until about two years ago, I was someone who always carried HIV with me and now I have kind of left it behind. I don't carry it with me anymore. I can laugh now. I can make jokes. I can feel good on my own if I want to. I think I have mourned enough. I've given it a place and if I want to go there, I do, and if I don't want to do that, I don't. (HIV-positive Antillean man)

A final emotion-focused coping strategy observed was acceptance that stigmatization is bound to happen. One participant said, "If I accept the fact that I have HIV, I also need to accept that which comes with it, and that is what I do. It's hard to accept but you have to" (HIV-positive Surinamese woman). Another said, "I had to go through [stigmatizing reactions]. There was no other way. You just have to fall down and push yourself up again" (HIV-positive Rwandan woman).

\section{DISCUSSION}

This study is one of the first to document, in detail, how HIV-related stigma manifests in African and Afro-Caribbean diaspora communities, what the consequences of such stigma is for the psychological well-being, social lives, and health of these PLWH, and how these $\mathrm{PLWH}$ respond to and cope with stigmatizing experiences.

Our findings show that HIV-related stigma manifests as social distance in the forms of avoidance, rejection, abandonment, and exclusion; physical distance from PLWH and objects they come in contact with; words through gossip, blaming, negative remarks, and disdain; and through silence and denial. These findings are corroborated by research conducted with African and Afro-Caribbean people in diaspora communities (M. Anderson et al., 2008; Dodds et al., 2004; Gardezi et al., 2008; Kinniburgh, Scott, Gottlieb, \& Power, 2001) and in their countries of origin (Bond, Chase, \& Aggleton, 2002; Duffy, 2005; VarasDiaz, Serrano-Garcia, \& Toro-Alfonso, 2005). For example, in research conducted by Kinniburgh and colleagues (2001), African PLWH in the United Kingdom reported being judged by their families and abandoned by their partner. They also reported a climate 
of secrecy regarding HIV in their community, as did Gardezi et al. (2008) in their study with African and Afro-Caribbean communities in Canada. Also, in a study conducted by Anderson and colleagues (2008), Jamaicans living in the United Kingdom reported gossip and verbal abuse by members of their community, and excessive protective measures (e.g. disinfecting clothes, forcing PLWH to use separate plates and silverware) by family members. Our finding that PLWH are excluded from preparing food and given separate eating utensils is particularly interesting in light of work done by Okoror and colleagues (2007) in South Africa. They documented how the separation of utensils and exclusion from food preparation and communal eating is an expression of rejection in cultures where food serves to establish and validate relationships and belonging. Thus, these forms of stigmatization not only reflect a fear of infection but also social rejection. Community members' concurrence with the manifestations reported by PLWH reinforces the fact that these manifestations permeate their culture and shape the treatment of PLWH.

With respect to the consequences of HIV-related stigma, our study has shown that PLWH experience emotional pain, sadness, loneliness, anger, and frustration because of stigmatizing experiences. Some PLWH also internalize the stigma they experience. These findings parallel those reported by Nyblade and colleagues (2003) who demonstrated that PLWH in Sub-Saharan Africa experience despondency, despair, and a loss of hope, and internalize stigma. The social consequences of HIV-related stigma reported in our study, namely decreased social network size, limited social support, and social isolation, were the result of not only enacted stigma but also self-imposed social withdrawal as a means of avoiding stigmatization. This is in line with the work of Smart Richman and Leary (2009) who claimed that chronic and pervasive rejection increases the likelihood of withdrawal and avoidance. In addition, we found that a particularly important context in which PLWH keenly feel the consequences of stigma was romantic relationships. This corresponds with Anderson et al. (2008) who found that African and Afro-Caribbean PLWH in the United Kingdom find it difficult to enter into and maintain long-term romantic relationships because of disclosure concerns and fears of rejection. A final consequence of HIV-related stigma reported in our study, pertaining to the health of PLWH, was poor treatment adherence. Similar difficulties with treatment adherence among migrant PLWH in the Netherlands were previously documented by Shiripinda and van Eerdewijk (2008).

Our study also elaborated coping strategies employed by African and Afro-Caribbean PLWH in the Netherlands and found that PLWH employed, often simultaneously, both problemfocused and emotion-focused coping strategies. The problem-focused coping strategies included selective disclosure or concealment, avoiding situations where stigmatization is likely (disengagement), affiliating oneself with similar others, seeking social support, and, 
to a lesser extent, activism. The emotion-focused strategies included distraction, positive reappraisal, religious coping, external attributions, disidentification with the stigmatized identity, and acceptance. These findings are line with the theoretical literature on coping with stigma (Crocker, Major, \& Steele, 1998; Miller \& Kaiser, 2001; Miller \& Major, 2000). They also correspond with work conducted with African and Afro-Caribbean PLWH. For example, Dodds and colleagues (2004) found that African PLWH in the United Kingdom cope by seeking support from other PLWH and their families. In the Caribbean, Carr (2004) found that PLWH cope with stigma by selectively disclosing, seeking social support from friends and family, and by turning to their faith. In Africa, problem-focused (e.g. connecting with other PLWH, seeking support, educating others) and emotion-focused coping responses (e.g. positive thinking, acceptance, religious coping) similar to those found in our study were comprehensively documented by Makoe and colleagues (2008).

Understanding which coping strategies PLWH employ is particularly important because some coping strategies mitigate the negative consequences of HIV-related stigma better than others do. For example, coping strategies such as support seeking and positive reappraisal have been found to be positively related to psychological well-being while stigma avoidance has been found to yield greater psychological distress (Gonzalez, Solomon, Zvolensky, \& Miller, 2009; Kraaij et al., 2008). Identifying which strategies PLWH use is the first step toward the development of interventions that seek to help PLWH use better coping strategies. Naturally, the onus for stigma reduction does not lie solely with PLWH. It would be unethical to limit interventions to training PLWH in how they can best cope with stigmatizing experiences. Interventions in the community that tackle the manifestations of HIV-related stigma documented in this study (i.e. social and physical distance, words and silence) are imperative, and must occur alongside interventions with PLWH (see Bos, Schaalma, \& Pryor, 2008; L. Brown, Macintyre, \& Trujillo, 2003).

Our study has both strengths and limitations. The primary strength of our study is its inductive nature and the 'thick' description of the data presented. We believe that this kind of description contributes substantially to a better understanding of HIV-related stigma in African and Afro-Caribbean diaspora communities, and provides an impetus for stigma reduction interventions in these communities. A second strength is the rigor of our study. We sought to promote study quality and trustworthiness in a number of ways. First, in contrast to most other studies on stigma in African and Afro-Caribbean diaspora communities, we triangulated data across PLWH and community members. Second, we used digital voice recorders and verbatim transcriptions to enhance rigor, and maintained a decision trail. Finally, we sought analytic integrity by seeking concurrence with previous findings (theory triangulation) and by checking our findings with relevant stakeholders (Creswell, 2009; 
Polit \& Beck, 2010). However, given the qualitative nature of the findings, one must be cautious in generalizing these results to other populations. A limitation of our study is the representativeness of the sample. Although qualitative studies do not seek to achieve representativeness through randomization, many do endeavor to attain some degree of representativeness with regard to, for example, gender, educational attainment, and age. In our study, we struggled to recruit Antillean women, Antilleans with a lower level of education, and older African PLWH. Nonetheless, our study design was rooted in existing theory and evidence, and stakeholder checks confirmed our findings thus suggesting that, despite difficulties in acquiring a representative sample, the findings are reflective of the current state of affairs in African and Afro-Caribbean communities in the Netherlands.

In conclusion, we have found that HIV-related stigma permeates African and AfroCaribbean communities in the Netherlands, that stigma manifests in these communities through social and physical distance, words and silence, and that this stigma negatively affects the psychological well-being, social lives, and health of African and Afro-Caribbean PLWH. We have also found that these PLWH employ a number of problem-focused and emotion-focused coping strategies to reduce the negative impact HIV-related stigma has on their lives. 
MANIFESTATIONS, CONSEQUENCES, AND COPING 


\section{HIV STATUS DISCLOSURE AMONG HIV-POSITIVE AFRICAN AND AFRO-CARIBBEAN PEOPLE
IN THE NETHERLANDS AND AFRO-CARIBBEAN PEOPLE
IN THE NETHERLANDS}

This chapter has been published in similar form as:

Stutterheim, S. E., Shiripinda, I., Bos, A. E. R., Pryor, J. P., de Bruin, M., Nellen, J. F. J. B., Kok, G., Prins, J. M., \& Schaalma, H. P. (in press). HIV status disclosure among HIVpositive African and Afro-Caribbean people in the Netherlands. AIDS Care, 23(2). 


\section{ABSTRACT}

HIV status disclosure is often characterized as a dilemma. On the one hand, disclosure can promote health, social support, and psychological well-being. On the other, disclosure can lead to stigmatization, rejection, and other negative social interactions. Previous research has shown that HIV status disclosure is a reasoned process whereby the costs and benefits to oneself and to others are weighed. As such, understanding disclosure requires understanding the reasons for and against disclosure employed by people living with HIV (PLWH). In this study, disclosure among a population disproportionately affected by HIV in the Netherlands, namely African and Afro-Caribbean diaspora, was investigated. Reasons for nondisclosure were fear of stigmatization, previous negative experiences with disclosure, having observed the stigmatization of other PLWH, shame, the desire to protect others - particularly one's children and family - from stigmatizationby-association and/or worrying, and the belief that one's HIV status is a private matter. Participants reported disclosing because they were in a close and supportive relationship, disclosure led to emotional release, disclosure could lead to emotional or financial support, they felt a perceived duty to inform, and they had a desire to educate others about sexual risk-taking. The findings suggest that stigma plays an important role in disclosure decisions among these populations. They further point to a need for HIV-related stigma reduction interventions in African and Afro-Caribbean communities and culturally sensitive counseling for PLWH whereby caregivers do not automatically assume that disclosure is best but rather provide a safe environment in which the costs and benefits of disclosure can be weighed and strategies for disclosure can be developed, if perceived as beneficial by PLWH. 


\section{INTRODUCTION}

In the Netherlands, ethnic minorities are disproportionately affected by HIVIAIDS. In fact, among people living with HIV (PLWH) in the Netherlands, one in every five is of African or Caribbean origin (HIV Monitoring Foundation, 2008). Previous research has demonstrated that, because of HIV-related stigma, disclosure of HIV status is an important concern among PLWH of African and Caribbean origin, both in their home countries and among the diaspora (J. Anderson \& Doyal, 2004; Kumar, Waterman, Kumari, \& Carter, 2006; Visser, Neufeld, de Villiers, Forsyth, \& Makin, 2008).

The term stigma refers to a distinctive, discrediting characteristic that renders its bearer tainted, flawed, or inferior in the eyes of others (Bos, Kok, \& Dijker, 2001; Crocker, Major, \& Steele, 1998; Goffman, 1963; Jones et al., 1984). The origin of stigmatization lies in the cognitive representations of people who possess the stigmatized condition. These cognitive representations may trigger emotional and behavioral reactions from others that subsequently result in stigmatizing behavior such as avoidance, blaming, and exclusion (Bos, Schaalma, \& Pryor, 2008; Dijker \& Koomen, 2003; Stutterheim et al., 2009). Stigmatizing responses to PLWH are promoted by a number of perceptions including the perception that HIV is highly contagious, the perception that HIV is severe and fatal, and the perception that PLWH are personally responsible for having acquired HIV. HIV-related stigmatization is further exacerbated by the fact that HIV has traditionally been associated with certain forms of norm-violating behavior such as homosexuality, commercial sex work, and intravenous drug use (Bos, Dijker, \& Koomen, 2007; Herek, 1999).

Because of HIV-related stigma, PLWH face a dilemma of disclosure (Pachankis, 2007). On the one hand, disclosure can lead to stigmatization in the form of discrimination, rejection, and other negative social interactions (Black \& Miles, 2002; Landau \& York, 2004), and stigmatization has been found to be detrimental to PLWH's psychological well-being (Riggs, Vosvick, \& Stallings, 2007; Stutterheim et al., 2009; Vanable, Carey, Blair, \& Littlewood, 2006). On the other, disclosure can be beneficial. It has been found to promote treatment adherence (Chesney \& Smith, 1999), safe sex (Melchert \& Patterson, 1999; Serovich \& Mosack, 2003), social support provision (Bos, Kanner, Muris, Janssen, \& Mayer, 2009; Smith, Rossetto, \& Peterson, 2008), closeness in relationships (Herek \& Capitanio, 1996; Parsons, VanOra, Missildine, Purcell, \& Gomez, 2004), and psychological well-being (Derlega, Winstead, Oldfield, \& Barbee, 2003; Smart \& Wegner, 1999). Clearly, both advantages and disadvantages of disclosure have been established. 
So what determines whether one discloses or conceals one's HIV status? Previous research has contended that HIV status disclosure is a reasoned process whereby the perceived costs and benefits to oneself and to others are weighed. When PLWH consider the benefits to outweigh the costs, disclosure is highly probable. When the costs outweigh the benefits, concealment is more likely (Black \& Miles, 2002; Derlega, Winstead, Greene, Serovich, \& Elwood, 2004; Serovich, 2001; Valle \& Levy, 2009). As such, understanding disclosure patterns requires understanding PLWH's reasons for and against disclosure (Calin, Green, Hetherton, \& Brook, 2007). This has been studied extensively in North America and Europe but less extensively in Africa or the Caribbean (for an overview of the literature, see Table 4.1). Almost no studies exploring the reasons for and against disclosure among HIV-positive African or Caribbean diaspora have been conducted. One exception is a study conducted by Calin and colleagues (2007) who investigated disclosure among Black African PLWH in the United Kingdom. In their study, reasons for nondisclosure cited were fear of rejection, broken confidentiality, assumptions of promiscuity by family and friends, negative previous experiences with disclosure, and the desire to protect others, particularly family members, from worrying. Reasons for disclosure included knowing that one's current or previous sexual partner was at risk for infection, believing that one's partner had a right to know, and knowing that one's confidant is also HIV-positive. Participants also reported disclosing their status because they were in a close relationship with someone they could trust, because confiding brought relief, because disclosure could lead to emotional support, and because they felt the need to explain physical decline (Calin, Green, Hetherton, \& Brook, 2007).

Given the high prevalence of HIV among African and Afro-Caribbean diaspora, we believe that additional investigation of the reasons for and against disclosure employed by these PLWH is warranted, especially given that, to date, only one study has explored disclosure among African diaspora and no studies have investigated disclosure among Afro-Caribbean diaspora. Further, we contend that a better understanding of how HIVpositive African and Afro-Caribbean diaspora approach the issue of disclosure will enable professionals to better support these PLWH. Consequently, in this study, we explored the reasons for nondisclosure and disclosure employed by African, Dutch Antillean, and Surinamese immigrants living in the Netherlands. 


\section{METHODS}

Study participants were recruited in one of three ways: directly by the interviewers (snowball sampling), via an online recruitment announcement placed on the Dutch HIV Association website, or through folders distributed by the Humanitas Foundation and/or HIV nurses working in Dutch hospitals. Once recruited, participants were provided with information regarding the purpose of the study and the procedure by either a researcher or an interviewer. Informed consent was obtained and a monetary reward of $€ 30$ was provided. Approval for this study was granted by the Faculty of Psychology and Neuroscience's Ethics Committee at Maastricht University.

In total, $42 \mathrm{PLWH}$ of African ( $N=15)$, Dutch Antillean ( $N=11)$, or Surinamese $(N=17)$ descent (one participant identified himself as both Antillean and Surinamese) were interviewed in face-to-face semistructured interviews by trained peer interviewers or researchers (SS, IS, MB) in either Dutch or English, depending on the participant's preference. Interviews were conducted between January 2005 and May 2008. A structured protocol of theorybased open-ended questions with follow-up probes was employed in the interviews. Sociodemographic data were also collected and are displayed in Table 4.2. Interviewers were not aware of participants' disclosure status prior to the interview. This was considered advantageous as previous knowledge of disclosure status could generate interviewer bias and impact the direction of the conversation. All interviews were recorded with a digital voice recorder and transcribed verbatim.

Data were processed using QSR NVivo 2.0. Each transcript was read thoroughly while listening to the corresponding recording to identify emerging themes and establish categories to which text fragments were assigned. As coding occurred, categories and subcategories were linked to one another. All emergent categories were documented, as were changes made to the categories and the rationale for the changes made. Coding continued until saturation was evident and no new codes were formed. Following the withincase analyses of the individual transcripts, across-case analyses were conducted to identify the overarching themes relevant to the different ethnic groups and the sample as a whole. 


\section{Table 4.1: Overview of the literature on reasons for and against disclosure of HIV status}

\begin{tabular}{|c|c|}
\hline Reasons for disclosure & Author(s), year, and study sample \\
\hline $\begin{array}{l}\text { Being in a close and } \\
\text { supportive relationship }\end{array}$ & $\begin{array}{l}\text { Calin et al., } 2007 \text { (African immigrants, UK); Derlega et al., } 2004 \text { (USA); } \\
\text { Gorbach et al., } 2004 \text { (MSM, USA); Sachperoglou \& Bor, } 2001 \text { (Greece); } \\
\text { Simoni et al., } 1995 \text { (Women, USA); Visser et al., (Women, South Africa) }\end{array}$ \\
\hline Emotional catharsis & $\begin{array}{l}\text { Calin et al., } 2007 \text { (African immigrants, UK); Derlega et al., } 2004 \text { (USA); } \\
\text { Ostrom et al. } 2006 \text { (Women, USA); Serovich, } 2001 \text { (MSM, USA); Serovich } \\
\text { \& Mosack, } 2003 \text { (MSM, USA); Valle \& Levy, } 2009 \text { (African American IDU, } \\
\text { USA) }\end{array}$ \\
\hline $\begin{array}{l}\text { Disclosure could lead to } \\
\text { emotional support }\end{array}$ & $\begin{array}{l}\text { Black \& Miles, } 2002 \text { (African American women, USA); Calin et al., } 2007 \\
\text { (African immigrants, UK); Chandra, Deepthivarma \& Manjula, } 2003 \\
\text { (India); Derlega et al., } 2004 \text { (USA); Ford et al., } 2004 \text { (Indonesia); Greeff } \\
\text { et al., } 2008 \text { (Sub-Saharan Africa); Ostrom et al. } 2006 \text { (Women, USA); } \\
\text { Serovich, } 2001 \text { (MSM, USA); Simoni et al., } 1995 \text { (Women, USA); Valle } \\
\text { \& Levy, } 2009 \text { (African American IDU, USA); Yoshioka \& Schustack, } 2001 \\
\text { (Asian Americans, USA) }\end{array}$ \\
\hline $\begin{array}{l}\text { Disclosure could lead } \\
\text { to instrumental/practical } \\
\text { support }\end{array}$ & $\begin{array}{l}\text { Greeff et al., } 2008 \text { (Sub-Saharan Africa); Ostrom et al. } 2006 \text { (Women, } \\
\text { USA); }\end{array}$ \\
\hline $\begin{array}{l}\text { Disclosure could lead } \\
\text { to financial/instrumental } \\
\text { support }\end{array}$ & $\begin{array}{l}\text { Chandra, Deepthivarma \& Manjula, } 2003 \text { (India); Valle \& Levy, } 2009 \\
\text { (African American IDU, USA) }\end{array}$ \\
\hline Perceived duty to inform & $\begin{array}{l}\text { Calin et al., } 2007 \text { (African immigrants, UK); Chandra, Deepthivarma \& } \\
\text { Manjula, } 2003 \text { (India); Derlega et al., } 2004 \text { (USA); Gorbach et al., } 2004 \\
\text { (MSM, USA); Holt et al., } 1998 \text { (UK); Ostrom et al. } 2006 \text { (Women, USA); } \\
\text { Parsons et al., } 2004 \text { (IDU, USA); Petrak et al., } 2001 \text { (UK); Serovich, } 2001 \\
\text { (MSM, USA); Serovich \& Mosack, } 2003 \text { (MSM, USA); Siegel, Lekas \& } \\
\text { Schrimshaw, } 2005 \text { (Women, USA); Simoni et al., } 1995 \text { (Women, USA); } \\
\text { Sowell et al., } 2003 \text { (Women, USA); Visser et al., } 2008 \text { (Women, South } \\
\text { Africa) }\end{array}$ \\
\hline $\begin{array}{l}\text { Desire to educate } \\
\text { regarding sexual risk } \\
\text { taking }\end{array}$ & $\begin{array}{l}\text { Black \& Miles, } 2002 \text { (African American women, USA); Derlega et al., } 2004 \\
\text { (USA); Frye et al., } 2009 \text { (IDU, USA); Greeff et al., } 2008 \text { (Sub-Saharan } \\
\text { Africa); Paxton, } 2002 \text { (Africa \& Pacific Asia); Petrak et al., } 2001 \text { (UK); } \\
\text { Serovich \& Mosack, } 2003 \text { (MSM, USA); Visser et al., } 2008 \text { (Women, } \\
\text { South Africa) }\end{array}$ \\
\hline To explain physical decline & $\begin{array}{l}\text { Calin et al., } 2007 \text { (African immigrants, UK); Greeff et al., } 2008 \text { (Sub- } \\
\text { Saharan Africa); Visser et al., } 2008 \text { (Women, South Africa) }\end{array}$ \\
\hline \multicolumn{2}{|l|}{$\begin{array}{l}\text { Reasons for } \\
\text { nondisclosure }\end{array}$} \\
\hline $\begin{array}{l}\text { Fears stigmatization/ } \\
\text { discrimination/negative } \\
\text { reactions }\end{array}$ & $\begin{array}{l}\text { Chandra, Deepthivarma \& Manjula, } 2003 \text { (India); Holt et al., } 1998 \text { (UK); } \\
\text { Kumar et al., } 2006 \text { (Women, Barbados); Parsons et al., } 2004 \text { (IDU, USA); } \\
\text { Petrak et al., } 2001 \text { (UK); Schrimshaw \& Siegel, } 2003 \text { (USA); Steward et } \\
\text { al., } 2008 \text { (India); Valle \& Levy, } 2009 \text { (African American IDU, USA) }\end{array}$ \\
\hline
\end{tabular}




\section{Reasons for} nondisclosure

Specifically...

Rejection/ abandonment

Being blamed for HIV infection

Gossip/breaches of confidentiality

Abuse

Previous disclosures have led to stigmatization

Has observed stigmatization of other PLWH

Feels shame

Wants to protect others from courtesy stigma

Wants to protect others from worrying

HIV status is a private matter

Disclosure is futile

The relationship is superficial

Communication difficulties
Author(s), year, and study sample

Calin et al., 2007 (African immigrants, UK); Derlega et al., 2004 (USA); Ford et al., 2004 (Indonesia); Frye et al., 2009 (IDU, USA); Gorbach et al., 2004 (MSM, USA); Greeff et al., 2008 (Sub-Saharan Africa); Levy et al., 1999 (France); Serovich, 2001 (MSM, USA); Serovich \& Mosack, 2003 (MSM, USA); Siegel, Lekas \& Schrimshaw, 2005 (Women, USA); Simoni et al., 1995 (Women, USA); Valle \& Levy, 2009 (African American IDU, USA); Visser et al., 2008 (Women, South Africa); Zea et al., 2003 (Latino MSM, USA)

Calin et al., 2007 (African immigrants, UK); Greeff et al., 2008 (SubSaharan Africa); Serovich, 2001 (MSM, USA); Valle \& Levy, 2009 (African American IDU, USA); Visser et al., 2008 (Women, South Africa)

Calin et al., 2007 (African immigrants, UK); Greeff et al., 2008 (SubSaharan Africa); Petrak et al., 2001 (UK); Serovich \& Mosack, 2003 (MSM, USA); Siegel, Lekas \& Schrimshaw, 2005 (Women, USA)

Ford et al., 2004 (Indonesia); Valle \& Levy, 2009 (African American IDU, USA); Visser et al., 2008 (Women, South Africa)

Black \& Miles, 2002 (African American women, USA); Calin et al., 2007 (African immigrants, UK); Greeff et al., 2008 (Sub-Saharan Africa)

Black \& Miles, 2002 (African American women USA); Frye et al., 2009 (IDU USA)

Black \& Miles, 2002 (African American women USA); Derlega et al., 2004 (USA); Serovich \& Mosack, 2003 (MSM USA); Simoni et al., 1995 (Women USA)

Black \& Miles, 2002 (African American women USA); Chandra, Deepthivarma \& Manjula, 2003 (India); Greeff et al., 2008 (Sub-Saharan Africa); Ostrom et al. 2006 (Women USA); Yoshioka \& Schustack, 2001 (Asian Americans USA)

Black \& Miles, 2002 (African American women USA); Calin et al., 2007 (African immigrants, UK); Derlega et al., 2004 (USA); Ostrom et al. 2006 (Women USA); Petrak et al., 2001 (UK); Serovich, 2001 (MSM USA); Simoni et al., 1995 (Women USA); Valle \& Levy, 2009 (African American IDU USA); Vallerand et al., 2005 (Women USA); Waugh, 2003 (UK)

Derlega et al., 2004 (USA); Gorbach et al., 2004 (MSM USA); Ostrom et al. 2006 (Women USA); Schrimshaw \& Siegel, 2003 (USA); Serovich \& Mosack, 2003 (MSM USA); Siegel, Lekas \& Schrimshaw, 2005 (Women USA); Valle \& Levy, 2009 (African American IDU USA)

Chandra, Deepthivarma \& Manjula, 2003 (India);

Derlega et al., 2004 (USA); Serovich \& Mosack, 2003 (MSM USA); Visser et al., 2008 (Women South Africa)

Derlega et al., 2004 (USA); Schrimshaw \& Siegel, 2003 (USA); Serovich \& Mosack, 2003 (MSM USA); Visser et al., 2008 (Women South Africa)

MSM = Men who have sex with men

IDU= Injecting drug users 
Table 4.2: Demographic and background characteristics of sample $(N=42)$

\begin{tabular}{|c|c|c|c|}
\hline Variable & $\begin{array}{r}\% \text { African } \\
(N=15)\end{array}$ & $\begin{array}{r}\% \text { Antillean } \\
(N=11)\end{array}$ & $\begin{array}{r}\% \text { Surinamese } \\
(N=17)\end{array}$ \\
\hline \multicolumn{4}{|l|}{ Gender } \\
\hline Male & 43.7 & 70.0 & 47.1 \\
\hline Female & 56.3 & 30.0 & 52.9 \\
\hline \multicolumn{4}{|l|}{ Age } \\
\hline Years of age (mean, SD) & $35.6(8.5)$ & $43.8(10.2)$ & $37.8(11.8)$ \\
\hline Range (min-max) & $18-51$ & $27-62$ & $22-70$ \\
\hline \multicolumn{4}{|l|}{ Level of Education* } \\
\hline Low & 18.8 & 30.0 & 23.5 \\
\hline Moderate & 50.0 & 30.0 & 53.0 \\
\hline High & 31.2 & 40.0 & 23.5 \\
\hline \multicolumn{4}{|l|}{ Employment/Income } \\
\hline Full-time work & 18.8 & 33.4 & 25.0 \\
\hline Part-time work & 12.5 & 22.2 & 0.0 \\
\hline Receiving benefits & 43.8 & 22.2 & 58.4 \\
\hline Studying & 6.2 & 0.0 & 8.3 \\
\hline Volunteer Work & 6.2 & 0.0 & 0.0 \\
\hline Combination & 12.5 & 22.2 & 8.3 \\
\hline \multicolumn{4}{|l|}{ Marital Status } \\
\hline Married & 20.0 & 0.0 & 13.3 \\
\hline Common law & 13.3 & 0.0 & 13.3 \\
\hline Single & 53.4 & 90.0 & 53.3 \\
\hline Divorced & 0.0 & 0.0 & 13.3 \\
\hline Widowed & 13.3 & 10.0 & 6.8 \\
\hline \multicolumn{4}{|l|}{ Sexual Orientation } \\
\hline Heterosexual & 80.0 & 40.0 & 68.8 \\
\hline Homosexual & 20.0 & 60.0 & 31.2 \\
\hline \multicolumn{4}{|l|}{ Transmission Mode } \\
\hline Sex & 81.3 & 100.0 & 93.8 \\
\hline Blood transfusion & 12.5 & 0.0 & 6.2 \\
\hline Other & 6.2 & 0.0 & 0.0 \\
\hline \multicolumn{4}{|l|}{ Time since diagnosis } \\
\hline Less than 2 years & 20.0 & 0.0 & 13.3 \\
\hline 2 to 4 years & 20.0 & 20.0 & 13.3 \\
\hline 4 to 6 years & 20.0 & 30.0 & 20.0 \\
\hline 6 to 8 years & 0.0 & 0.0 & 33.4 \\
\hline 8 to 10 years & 20.0 & 30.0 & 0.0 \\
\hline more than 10 years & 20.0 & 20.0 & 20.0 \\
\hline
\end{tabular}




\begin{tabular}{lrrr}
\hline Variable & $\begin{array}{r}\text { \% African } \\
(N=15)\end{array}$ & $\begin{array}{r}\% \text { Antillean } \\
(N=11)\end{array}$ & $\begin{array}{r}\% \text { Surinamese } \\
(N=17)\end{array}$ \\
\hline $\begin{array}{c}\text { Treatment } \\
\text { Antiretroviral therapy }\end{array}$ & 60.0 & 85.7 & 100.0 \\
$\quad$ No therapy & 40.0 & 14.3 & 0.0 \\
Current Health & & & \\
$\quad$ Very poor & 6.7 & 0.0 & 11.1 \\
$\quad$ Poor & 13.3 & 0.0 & 0.0 \\
Reasonable & 6.7 & 14.3 & 33.3 \\
Good & 26.7 & 42.8 & 44.5 \\
$\quad$ Very good & 33.3 & 14.3 & 0.0 \\
$\quad$ Excellent & 13.3 & 28.6 & 11.1 \\
\hline
\end{tabular}

Percentage of those participants with valid data; in most cases, missing data did not exceed $10 \%$; on some sensitive topics such as means of transmission, treatment, and current health, missing data exceeded $10 \%$.

*Low = less than high school; moderate = high school and some vocational training; high = college or university degree

\section{RESULTS}

\section{Disclosure targets}

Participants reported a number of disclosure targets including immediate family, extended family, sexual or romantic partners, friends, and colleagues or management at work. Although the qualitative methods and approach employed in this study were not designed to establish estimates of disclosure prevalence, the data did suggest that partners and immediate family members are the most common targets for disclosure, followed by friends and extended family. Disclosure at work was infrequent.

\section{Reasons for nondisclosure}

Perhaps the most prevalent reason reported for concealing one's HIV status was fear of stigmatization. In fact, many participants indicated that, in the past, they had personally experienced stigmatization following the disclosure of their HIV status and did not want to experience these kinds of reactions again. One Surinamese woman stated, "I have had a couple of bad experiences and I don't want that again." Another Rwandan woman said, "I did not want to go down that road again." Also, a number of men who have sex with men 
(MSM) referred to poor experiences related to coming out about their sexual orientation, and stated that they expected similar reactions to an HIV status disclosure: The following comments of a Surinamese MSM illustrate this.

Participant: I have seen how people reacted to my homosexuality and I thought, "Wow!"

Interviewer: Was it very negative?

Participant: From some colleagues, it was, but it wasn't directed straight at me. I just heard how they talked about it after the fact. One guy told another and that guy told another. I just think, "Wow, if they talk about gays that way, what would they say about HIV?" So I just decided l'd rather not have that.

Additionally, a number of participants indicated that they would rather keep their status concealed after seeing how other PLWH are stigmatized. One Antillean man stated, "Now they don't know about me but I have heard how they talk about other people who have HIV, like 'Have you seen him?' I'd rather not go through that."

In fact, the PLWH in our study were keenly aware of the potential stigmatizing reactions they may experience if they choose to disclose. Some participants mentioned thinking that others will reject, avoid, or abandon them. A Ugandan woman stated, "If I come and tell you, you'll just throw me out. I keep quiet." An Antillean man further illustrated this: "I don't dare talk about my HIV because I am scared to be rejected." Another form of stigmatization feared was gossip and breaches of confidentiality. Gossip was a major concern given the fact that the diaspora communities in the Netherlands are relatively small and that members are well-connected. The following excerpt from an interview with a young Zambian woman illustrates this fear of gossip.

You can't trust people! Definitely don't tell other people because if she tells another - one person "Don't talk", [that person] will go and tell another one, and they will go to another one [saying,] "Don't you say it but that one is positive".

Some participants also claimed that disclosure would lead to blaming. One Surinamese man said, "I know exactly how they think in those circles, and I don't feel like being confronted with this whole thing like that maybe it's because of my 'sins'. I don't want a whole sermon on it." This fear of blaming appeared to be particularly salient among participants with religious families, perhaps reflecting how the prominence of the Christian faith in these diaspora communities impacts PLWH's sense of acceptance by others, of rather, lack thereof. 
Another reason cited for nondisclosure was shame. When asked why she has not told people that she is HIV-positive, one woman from Cameroon answered, "Because of the shame it carries, the disgrace it carries." Another male participant from Suriname said, "I'm ashamed of it. I am ashamed that I have HIV. I don't tell people. Those who know, know. I am just so ashamed of it." This reflects what is termed internalized stigma. Internalized stigma occurs when a person possessing the stigmatized condition internalizes society's negative views about that condition (R. S. Lee, Kochman, \& Sikkema, 2002).

Yet another reason for nondisclosure related to the impact disclosure could have on others. Many participants reported not wanting to disclose because it may result in stigmatizationby-association toward, particularly, their children. Stigmatization-by-association, also termed courtesy stigma, is a phenomenon whereby the negative attitudes toward a stigmatized person are extended to people associated with that person (Goffman, 1963; Neuberg, Smith, Hoffman, \& Russell, 1994). One African participant said she would not tell others, "especially for my son. They will point at him and I don't want that." Another woman from the Dutch Antilles said, "Your kids suffer. Everyone will tell the other kids and then my kids won't have friends anymore. No, leave that problem to me. Let me experience it all, not my kids. I want them to have a good childhood." Again, concerns regarding the potential for stigmatization-by-association appeared to be particularly relevant given the small and well-connected nature of the diaspora communities.

Also, many participants conveyed opting to conceal their status so as to spare others, particularly their parents and children, from pain and from being worried. One Antillean man said, "I have told a few of my closest friends but not my parents because I think it might be too much for them." Another Kenyan man described his decision to not tell his parents as follows:

Well, when I knew that I was doing good, I thought it is not really a good idea to tell my parents because it might give them stress, especially when you are the one that's prayed for. You will worry them so I thought it is good I keep it like that [quiet].

Another participant, originally from Suriname, conveyed that she did not want to tell her children because of "the grief it would bring them, that they would not be able to go on with their lives, that they would be too concerned, and that they would think, 'mom is going to die soon."”

A final reason provided for nondisclosure was the right to privacy. A number of participants indicated that they felt no need or obligation to share their status while they are still healthy 
and able to function. One Ugandan man stated, “I don't see any reason to tell them yet that this is the situation. I don't tell them when I have a headache or stub my toe, cross the street, etc. Likewise, I don't feel it is valuable to tell."

\section{Reasons for disclosure}

Participants also conveyed a number of reasons in favor of disclosure. The first has to do with the nature of the relationship between the PLWH and the person to whom they disclosed. Participants who felt they were in a close, trusting relationship also felt that disclosure to that person was appropriate. One Kenyan man stated, "I tell them - the ones that I trust. You can't say it to anybody but you have some people sometimes you trust." Another Antillean man said, "I told people I could trust - people I have a connection with." Yet another participant from Suriname, when asked why he told particular people, responded, "Because I have a good relationship with them."

Another reason for disclosure was emotional catharsis. A number of participants reported no longer wanting to keep their "secret" to themselves. One Antillean man said, "When I kept it to myself, I didn't feel good. I didn't feel good because I was keeping this secret and it just makes you depressed." Another man, who is both Surinamese and Antillean, said, "It was too much. You just feel caged and then you need to vent so you make a choice: 'I'm going to tell it now."' Also, a Zambian woman indicated that she had told her sister because "it was so terrible to me so I can't hold it."

An additional reason for disclosure was the need for social, instrumental, and/or financial support. One Surinamese participant stated, "I felt that they needed to know because it is a difficult thing. I don't know how to say it ... I just thought they needed to know so that they know what I am dealing with." Another woman from Zimbabwe reported having told some family members in order to ensure support for her children.

What if something happens to me now? Who would look after my kids? Then you start thinking of your relatives and say, "I think this person could really take care of my kids," and then, in that way, you really confide in them, and then you tell them.

Yet another reason for disclosure provided was that the PLWH felt a sense of responsibility or duty to inform new sexual partners. Many participants reported feeling compelled to share their status with new sexual partners. One Antillean participant said, "I tell it right away and they can decide if they want to get involved with me." This sense of responsibility and duty is also exemplified by the following comment from an African woman. 
I have a new boyfriend. We have talked and I am working on telling him. He wants a relationship with me and wants us to be honest. He's told me a few things about himself and I should tell him.

The final noteworthy justification for HIV status disclosure was the desire to educate others. A number of participants reported that they would be willing to be open about their HIV status if it would contribute to decreasing others' sexual risk-taking. One young Surinamese woman said, "I know that she [a friend] is kind of easy with the boys and stuff so I told her because it is not something you expect. You don't think you can get HIV." Another Antillean participant said, "If I know that you will do something with the information, I'll tell you."

\section{DISCUSSION}

Although previous studies have established a number of reasons for and against disclosure employed by PLWH in Western countries and, to some extent, the developing world (see Table 4.1), this study is, to our knowledge, one of the first to explore how HIVpositive African and Afro-Caribbean diaspora approach decisions to disclose. In our study, the reasons for nondisclosure reported were fear of stigmatization, previous negative experiences with disclosure, having observed the stigmatization of other PLWH, shame, the desire to protect others - particularly one's children and family - from stigmatizationby-association and/or worrying, and the belief that one's HIV status is a private matter. Participants reported disclosing because they were in a close and supportive relationship, disclosure led to emotional release, disclosure could lead to emotional or financial support, they felt a perceived duty to inform, and they had a desire to educate others about sexual risk-taking. These results are very much in line with the work of Calin and colleagues (2007) who studied disclosure among Black African PLWH in the United Kingdom.

Our findings, as well as those of Calin et al. (2007), suggest that stigma plays an important role in disclosure decisions among African and Afro-Caribbean diaspora. In our study, participants not only cited fear of direct stigmatization and stigmatization-by-association as reasons for nondisclosure; they also reported personal experiences with enacted stigma, shame (i.e. internalized stigma), and the stigmatization of other PLWH as justifications for HIV status concealment. This is not surprising given that high levels of stigma have been reported in African and Caribbean countries (M. Anderson et al., 2008; Kalichman \& Simbayi, 2004). In a study conducted with PLWH in Sub-Saharan Africa, Greeff and colleagues (2008) found that refusal to disclose was a result of seeing how other PLWH had been treated. Also, participants cited the effect disclosure could have on the family 
(i.e. being shunned and stigmatized) as a reason for concealment. In a study conducted in Barbados, $30 \%$ of participants that had only disclosed their HIV status to health care providers indicated fear of stigmatization as the reason for nondisclosure to others. An additional $23 \%$ indicated not disclosing to their current sexual partner in order to avoid abnormal reactions and possible violence (Kumar, Waterman, Kumari, \& Carter, 2006). Clearly, our findings with African and Afro-Caribbean diaspora in the Netherlands are congruent with research results from studies conducted in Africa and the Caribbean.

Previous research has contended that cultural values may play an important role in disclosure-related decision-making (Simoni et al., 1995; Yoshioka \& Schustack, 2001). Interestingly, our analyses did not lead us to believe that the reasons employed by African and Afro-Caribbean diaspora in the Netherlands differed substantially from those employed by North American and European PLWH or by PLWH in other parts of the world. In fact, many of the reasons for and against disclosure cited by the participants in our study have also been found in studies conducted not only in the countries from which our participants originated but also elsewhere (for an overview of the literature, see Table 4.1). Perhaps it is not the case that cultural values generate different reasons for and against disclosure but rather that cultural values impact the importance assigned to a given reason. It may be that in some cultures some reasons weigh more heavily than others. For example, among Asian PLWH, fear of bringing shame to the family and thus the desire to protect family from stigmatization-by-association may weigh heavier than other reasons (Yoshioka \& Schustack, 2001). Among African PLWH, who have likely been subjected to or observed more stigmatization than many other PLWH, fear of stigmatization may weigh heavier than other reasons (Greeff et al., 2008). In our study, we found that fear of gossip and stigmatization-by-association were particularly salient reasons for nondisclosure because of the relatively small and well-connected nature of diaspora communities. We recommend that future research follow this qualitative study with quantitative investigations of, firstly, the relative importance of each of the reasons for and against HIV status disclosure across communities and cultures and, secondly, the impact of the relative weighting of reasons on actual disclosure.

Some limitations to this study should be mentioned. First, given the qualitative nature of the data, caution should be applied in generalizing the results of this study to other populations. Second, despite efforts to interview a relatively equal number of men and women per group and to have representative participants in terms of educational attainment, age, and sexual orientation, in this study, Antillean women and older Africans were underrepresented while highly educated Antilleans and gay Antillean men were overrepresented. Nonetheless, the reasons cited in this study appeared relatively consistent across all participants thus 
suggesting that these themes cut across the boundaries of gender, age, education, and sexual orientation. Third, there is potential for interviewer bias in this study. This, however, was combated insofar as possible by the use of digital voice recordings and verbatim transcriptions (Hancock, 1998).

Our finding that stigmatization impedes HIV status disclosure has important practical implications. Firstly, it points to the need for culturally sensitive counseling for PLWH of African and Afro-Caribbean descent. We contend that, given the high prevalence of stigma in African and Caribbean communities (M. Anderson et al., 2008; Kalichman \& Simbayi, 2004), health care providers should not automatically assume that disclosure of HIV status is best. Rather, they should consider the cultural context in which disclosure occurs and the potential for negative reactions to HIV status disclosure. In their efforts to support African and Afro-Caribbean PLWH, we recommend that health care providers use their unique role to provide these PLWH with a safe environment in which the costs and benefits of disclosure can be weighed and strategies for effective disclosure can be developed, if perceived as beneficial by PLWH. A supportive and safe environment for PLWH is imperative (Bos, Schaalma, \& Pryor, 2008; Nyblade, Pande, Mathur, MacQuarrie, \& Kidd, 2003) and can be promoted not only in health care providers' offices but also in the communities in which HIV-related stigma is so prevalent. We recommend culturally appropriate theory and evidence-based HIV-related stigma reduction interventions that tackle a number of manifestations of stigma across a broad range of settings within and beyond African and Afro-Caribbean communities (see Bos, Schaalma, \& Pryor, 2008; L. Brown, Macintyre, \& Trujillo, 2003; Stutterheim et al., 2009). Also, given that some participants conveyed negative experiences with disclosure in the past, we recommend the implementation of skill-building interventions that aid and support PLWH to disclose in ways that are most advantageous and least likely to generate negative responses (see Bos, Dijker, \& Koomen, 2007). Further, structural supports for PLWH (e.g. ongoing counseling programs, support groups) can also contribute to more advantageous disclosures of HIV status and ameliorate the negative impact of HIV-related stigma experiences. 


\section{HIV-RELATED STIGMA AND PSYCHOLOGICAL DISTRESS: \\ THE HARMFUL EFFECTS OF SPECIFIC STIGMA MANIFESTATIONS IN VARIOUS SOCIAL SETTINGS}

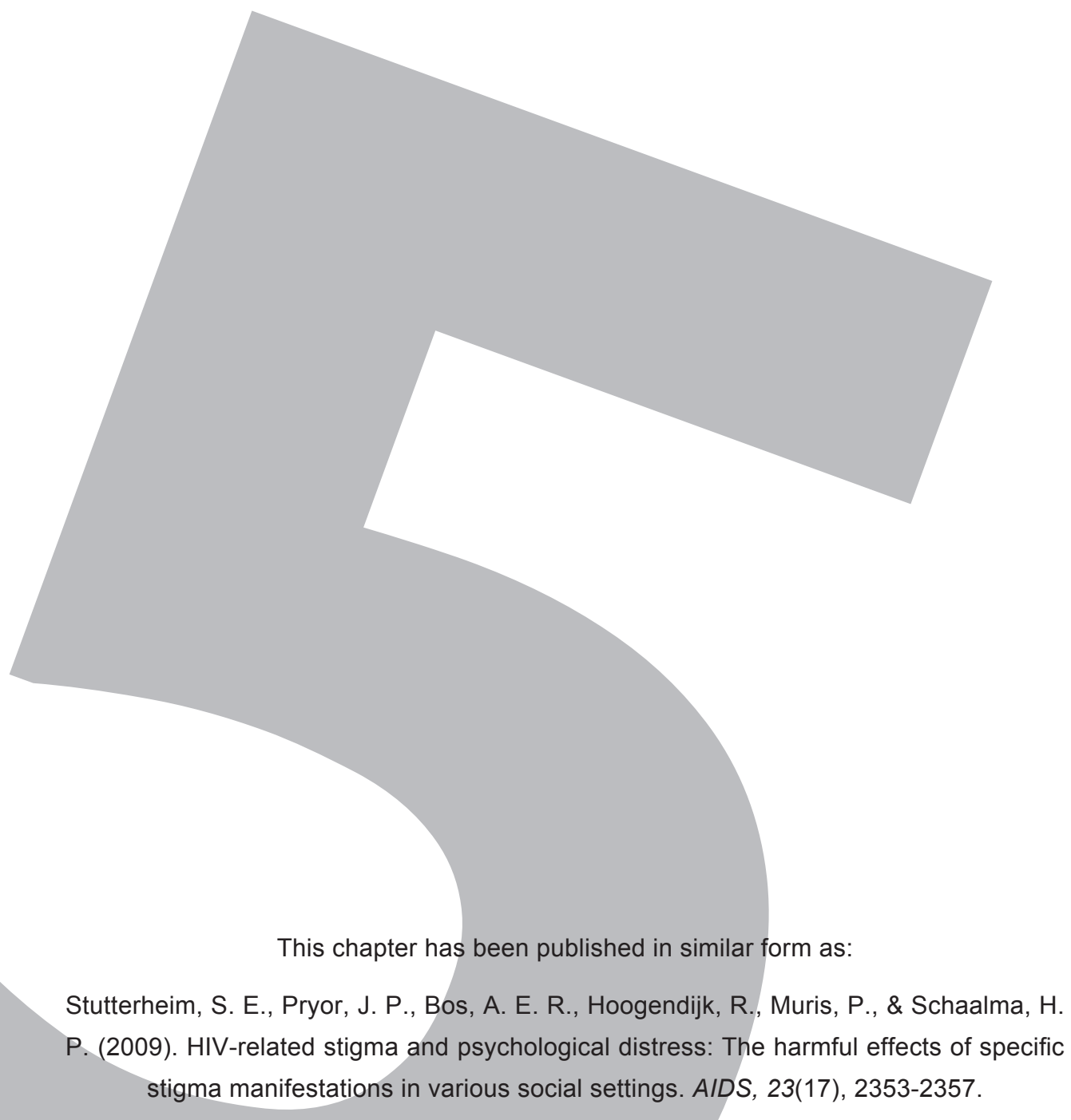




\section{ABSTRACT}

Recent research has shown that experiences of stigmatization have an adverse impact on the psychological well-being of people living with HIV (PLWH). Most studies investigating this relationship employ an aggregate measure of stigma. Although this approach provides useful information about the psychological implications of HIV-related stigma in general, it neglects to acknowledge the possibility that some manifestations in specific settings may be psychologically more detrimental than others. The present study examines which specific stigma experiences are most strongly related to psychological distress across a number of social settings. A cross-sectional survey was administered to 667 PLWH in the Netherlands. We examined participants' experiences of 11 manifestations of HIV-related stigma in 6 social settings. Linear regression analyses were conducted to determine which setting-specific manifestations best predict psychological distress after controlling for marital status, education, and health status. Three manifestations in family settings, namely receiving advice to conceal one's status, being avoided, and being treated with exaggerated kindness, and one manifestation in health care settings, namely awkward social interaction, best predicted psychological distress in PLWH. Manifestations of HIVrelated stigma thus vary according to setting. These findings suggest that stigma reduction interventions focusing on these influential settings may benefit the psychological wellbeing of $\mathrm{PLWH}$. 


\section{INTRODUCTION}

HIV-related stigma is a social phenomena whereby a person is considered to possess a discrediting attribute and thus deemed tainted, spoiled, or flawed by others (Bos, Kok, \& Dijker, 2001; Crocker, Major, \& Steele, 1998; Goffman, 1963; Jones et al., 1984). HIVrelated stigma can hamper HIV prevention efforts (UNAIDS, 2008), inhibit treatment adherence (Chesney \& Smith, 1999; Herek, 1999; Nyblade, Pande, Mathur, MacQuarrie, \& Kidd, 2003), function as a barrier to HIV testing (Meiberg, Bos, Onya, \& Schaalma, 2008; Vermeer, Bos, Mbwambo, Kaaya, \& Schaalma, 2009), and negatively impact social relationships and the psychological well-being of PLWH (Bos, Schaalma, \& Pryor, 2008; Crandall \& Coleman, 1992; Pryor, Reeder, Yeadon, \& Hesson-McLnnis, 2004; Stutterheim, Bos, \& Schaalma, 2008). In fact, research conducted in various countries, including South Africa (Simbayi et al., 2007), China (Mak et al., 2007), Peru (Wu et al., 2008), and the USA (Clark, Lindner, Armistead, \& Austin, 2003; Kang, Rapkin, Remien, Mellins, \& Oh, 2005; R. S. Lee, Kochman, \& Sikkema, 2002; Riggs, Vosvick, \& Stallings, 2007; Vanable, Carey, Blair, \& Littlewood, 2006), has demonstrated that HIV-related stigma independently contributes to psychological distress over and above health status and HIV-related symptoms.

Stigmatizing reactions to PLWH manifest in a number of ways across a range of settings. Relevant manifestations include avoidance, exclusion, rejection, isolation, social ostracism, blaming, violence, service denial, physical distance, indifference, awkward social interaction, and being advised to conceal one's status (Bond, Chase, \& Aggleton, 2002; Carr \& Gramling, 2004; Gielen, McDonnell, Burke, \& O'Campo, 2000; Herek, 1999; Herek, Capitanio, \& Widaman, 2002; Malcolm et al., 1998; Rintamaki, Scott, Kosenko, \& Jensen, 2007; Sandelowski, Lambe, \& Barroso, 2004; Sayles, Ryan, Silver, Sarkisian, \& Cunningham, 2007; Swendeman, Rotheram-Borus, Comulada, Weiss, \& Ramos, 2006; Vanable, Carey, Blair, \& Littlewood, 2006; Varas-Diaz, Serrano-Garcia, \& ToroAlfonso, 2005). Relevant settings are families, communities, friends or acquaintances, sexual relationships, health care settings, the housing sector, the financial services sector, religious institutions, while travelling or migrating, work, and educational settings (Bermingham \& Kippax, 1998; Carr \& Gramling, 2004; Dray-Spira, Lert, Marimoutou, Bouhnik, \& Obadia, 2003; Green \& Platt, 1997; Herek, 1999; Herek, Capitanio, \& Widaman, 2002; Malcolm et al., 1998; Monico, Tanga, \& Nuwagaba, 2001; Sandelowski, Lambe, \& Barroso, 2004; Simbayi et al., 2007; Simoni, Mason, \& Marks, 1997; VarasDiaz, Serrano-Garcia, \& Toro-Alfonso, 2005). 
To our knowledge, no previous quantitative study has explored how particular manifestations in specific social settings impact the psychological well-being of PLWH. In fact, most studies investigating the psychological impact of HIV-related stigma employ an aggregate measure of stigma such as the HIV stigma scale (Berger, Ferrans, \& Lashley, 2001) and the AIDS-related stigma scale (Kalichman et al., 2005). Although this approach provides useful information about the psychological implications of HIV-related stigma in general, it neglects to acknowledge the possibility that some manifestations in specific settings may be psychologically more detrimental than others. The present study examines which specific stigma experiences are most strongly related to psychological distress across a number of social settings.

\section{METHODS}

\section{Participants and procedure}

All data were obtained from an anonymous national survey with PLWH in the Netherlands. Participation was voluntary, informed consent was provided, and no monetary compensation was involved. Following approval from Maastricht University's Ethics Committee, a total of 2,264 surveys were distributed by the Dutch HIV Association $(N=1433)$ and by HIV nurses $(N=823)$. The surveys distributed by the HIV Association were sent by mail to all members in May 2007 with a reminder letter four weeks later. The surveys distributed by HIV nurses were handed out to patients during consultations between June and September 2007. A total of 669 participants completed the survey (response=29.5\%). Of these, 468 were recruited by the HIV Association (response=32.7\%) and 193 by HIV nurses (response=23.5\%). Three participants contacted the researchers directly for a survey. For five others, data on how they were recruited was missing. Two surveys were excluded from the analyses as the corresponding participants were outliers with respect to age (6 and 97 years) thus yielding a total of 667 participants.

Of these $667,86.2 \%$ were male and $13.8 \%$ were female. Ages ranged from 17 to 75 with a mean age of $46.6(S D=9.6)$. Almost half $(49.5 \%)$ had at least a Bachelor's degree, $31.0 \%$ had a high school diploma and some vocational training, and $19.5 \%$ had a high school diploma or less. Furthermore, $68.3 \%$ had paid employment and $48.4 \%$ had a long term partner. The majority defined themselves as gay (79.5\%) and from Europe or North America (90.6\%). Most participants (87.5\%) had acquired HIV through sexual intercourse. The mean time since diagnosis was 8.75 years $(S D=6.0)$. 


\section{Measures}

HIV-related stigma was measured using an index developed by the authors (available upon request) following a review of the social stigma literature and a focus group with experts, PLWH, and service providers working with various PLWH populations in the Netherlands. This index measured 11 manifestations across 6 social settings. The manifestations were increased physical distance, awkward social interaction, indifference, avoidance, blaming, exaggerated kindness, aggression, exclusion, excessive hygienic measures, being told to disclose one's status, and being told to conceal one's status. The settings were friends, family, partner, health care sector, work, and leisure activities. The questions were formatted such that participants first indicated whether they had experienced a given manifestation. They then indicated the settings in which that manifestation occurred. Participants were permitted to mark more than one setting.

Psychological distress was measured using a validated version of the Mental Health Inventory (MHI) which measures depression, anxiety, positive affect, and behavioral control (Veit \& Ware, 1983). The scale comprises 18 items, all of which are answered on a six-point scale ranging from 1 (none of the time) to 6 (all of the time). A higher score is indicative of more psychological distress. Cronbach's alpha was .94.

Demographic and background characteristics were also documented. Demographic characteristics measured included gender, age, educational attainment, employment, marital status, sexual orientation, and ethnic background. Other background characteristics measured included the mode by which one acquired HIV, time since diagnosis, the presence of visible symptoms, current treatment with antiretroviral therapy, self-reported health status, and recruitment method.

\section{Data analyses}

After generating descriptive statistics, we determined whether settings differ with respect to the mean number of manifestations using a repeated measures analysis of variance and paired samples $t$-tests. For the $t$-tests, $p$ values $<.001$ were considered statistically significant. This was followed by a series of setting-specific linear regressions of psychological distress on the 11 manifestations. Covariates were determined by initially establishing which demographic and background characteristics were correlated with psychological distress. All significant demographic and background characteristics were then entered into an initial linear regression model. Those that remained significant, namely having a partner, educational attainment, and self-reported health status, were 
then included in the setting-specific regression analyses. Following these analyses, a final model was tested to determine which particular manifestation and setting combinations most strongly predict psychological distress. This model included only those predictors that were significant in the setting-specific regression models. For all regression models, $p$ values $<.05$ (two tailed) were considered statistically significant.

\section{RESULTS}

With respect to the general prevalence of stigma experiences (see Table 5.1), we found that while more than half of the participants (54.1\%) had been advised to conceal their HIV status, more than a quarter $(28.8 \%)$ had been encouraged to disclose their HIV status to others. Other important manifestations of HIV-related stigma were blaming $(38.2 \%)$, increased physical distance $(34.4 \%)$, avoidance $(30.9 \%)$, excessive hygienic measures $(29.5 \%)$, indifference $(28.7 \%)$, and exclusion $(27.3 \%)$. Also, one in every twelve PLWH $(8.6 \%)$ reported suffering some form of aggression as a result of their HIV status.

Repeated measures analysis of variance established that the mean number of stigma manifestations differed significantly across settings $F(1,634)=46.749, p<.001, \eta 2=.07$. Paired samples $t$-tests showed that the mean number of manifestations in the setting friends $(M=1.14, S D=1.67)$ was not significantly higher than with family $(M=0.96, S D=1.55)$ but was significantly higher than all other settings, all $t(634) s>4.11, p<.001$. Also, the settings family, health care sector $(M=0.87, S D=1.38)$, and work $(M=0.83, S D=1.48)$, which did not differ significantly from one another, all had significantly higher means than the settings partner $(M=0.41, S D=0.87)$ and leisure activities $(M=0.40, S D=1.11)$, all $t(634) s>6.83, p<.001$.

Six hierarchical linear regression analyses investigated the relationships between stigma experiences in each of the specific settings and psychological distress (see Table 5.2). Significant predictors were: 1) For friends: blame, awkward social interaction, and exaggerated kindness ( $p s<.05)$; 2) For family: being advised to conceal one's status, avoidance, and exaggerated kindness (ps<.01); 3) For the health care sector: indifference and awkward social interaction; and 4) For partner: being told to conceal and exaggerated kindness $(p s<.05)$. The overall leisure settings and work models produced significant $R^{2} s$ but did not identify specific manifestations predicting psychological distress.

The ten significant predictors from the setting-specific models were then entered into a final regression model (see Table 5.3) which yielded an $R^{2}$ change of .09 $(p<.001)$. Four 
significant predictors emerged: being told to conceal by family $(p<.01)$, being avoided by family $(p<.01)$, experiencing exaggerated kindness from family $(p<.05)$, and awkward social interaction in the health care sector $(p<.05)$.

\section{Table 5.1: Overall frequencies of HIV-related stigma manifestations} experienced by PLWHA

\begin{tabular}{lcc}
\hline & $\%$ & $N$ \\
\hline Told to conceal $(N=653)$ & 54.1 & 353 \\
Blame $(N=655)$ & 38.2 & 250 \\
Increased physical distance $(N=652)$ & 34.4 & 224 \\
Avoidance $(N=651)$ & 30.9 & 201 \\
Excessive hygienic measures $(N=648)$ & 29.5 & 191 \\
Told to disclose $(N=652)$ & 28.8 & 188 \\
Indifference $(N=648)$ & 28.7 & 186 \\
Exclusion $(N=653)$ & 27.3 & 178 \\
Awkward social contact $(N=648)$ & 19.8 & 128 \\
Exaggerated kindness $(N=655)$ & 19.4 & 127 \\
Aggression $(N=653)$ & 8.6 & 56 \\
\hline
\end{tabular}

Percentages represent the number of participants that reported having experienced the manifestation divided by the total number of participants that responded to the item regarding the manifestation.

\section{DISCUSSION}

Although previous studies have established that HIV-related stigma does indeed independently contribute to psychological distress in PLWH (Clark, Lindner, Armistead, \& Austin, 2003; Kang, Rapkin, Remien, Mellins, \& Oh, 2005; R. S. Lee, Kochman, \& Sikkema, 2002; Mak et al., 2007; Riggs, Vosvick, \& Stallings, 2007; Simbayi et al., 2007; Vanable, Carey, Blair, \& Littlewood, 2006; Wu et al., 2008), the present study is, to our knowledge, the first quantitative study to explore how specific manifestations of HIV-related stigma are associated with psychological distress across a number of social settings. Our findings suggest that certain setting-specific manifestations of stigma are indeed more psychologically damaging than others. Psychological distress was most strongly predicted by three specific manifestations of stigma occurring in family settings - avoidance, exaggerated kindness, and being told to conceal one's status - and one 


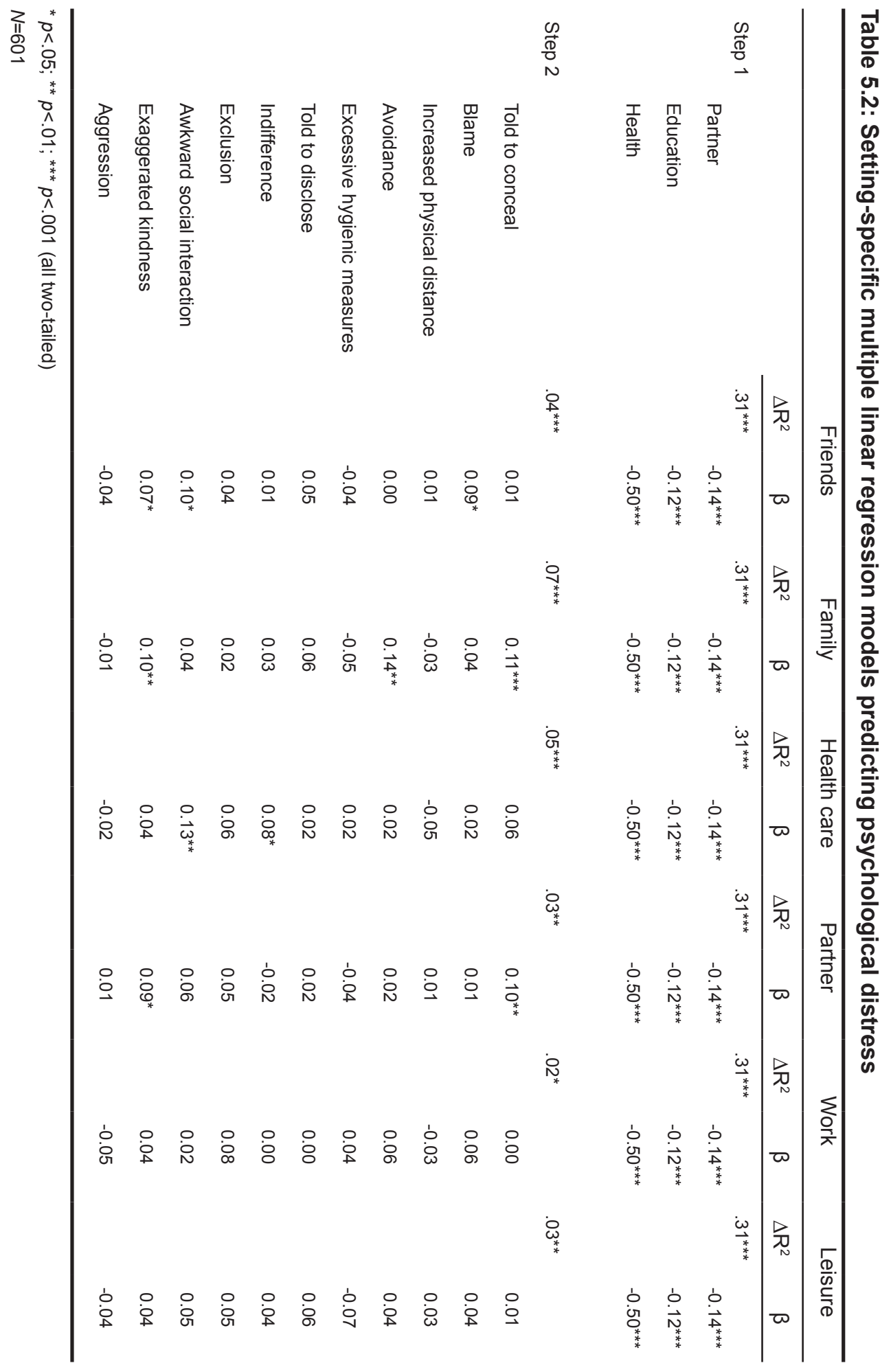


Table 5.3: Final multiple regression model predicting psychological distress

\begin{tabular}{|c|c|c|c|}
\hline & & $\Delta \mathrm{R}^{2}$ & $\beta$ \\
\hline \multirow[t]{4}{*}{ Step 1} & & $.31^{* * *}$ & \\
\hline & Partner & & $-0.14^{* * *}$ \\
\hline & Education & & $-0.12^{* * *}$ \\
\hline & Health & & $-0.50^{* * *}$ \\
\hline \multirow[t]{11}{*}{ Step 2} & & $.09^{* * *}$ & \\
\hline & Blaming (friends) & & 0.06 \\
\hline & Awkward social contact (friends) & & 0.05 \\
\hline & Exaggerated kindness (friends) & & 0.00 \\
\hline & Told to conceal (family) & & $0.09^{* *}$ \\
\hline & Avoidance (family) & & $0.10^{* *}$ \\
\hline & Exaggerated kindness (family) & & $0.08^{*}$ \\
\hline & Indifference (health care) & & 0.04 \\
\hline & Awkward social interaction (health care) & & $0.09^{*}$ \\
\hline & Told to conceal (partner) & & 0.06 \\
\hline & Exaggerated kindness (partner) & & 0.05 \\
\hline
\end{tabular}

${ }^{*} p<.05 ;{ }^{* *} p<.01 ;{ }^{* * *} p<.001$ (all two-tailed)

$N=601$

manifestation in health care settings - awkward social interaction. Stigmatization by family may be particularly detrimental as families are not chosen and often considered an important source of unconditional love and support. Stigmatization by family may thus threaten a fundamental human need, namely the need to belong (Baumeister, Leary, Higgins, \& Kruglanski, 2000). With respect to the impact of awkward social interactions, previous research has shown that many PLWH assume that health professionals are knowledgeable about HIV and thus expect them to be at ease with them (Green \& Platt, 1997). When health professionals' actions suggest otherwise, disappointment and subsequent psychological distress may ensue.

Our finding that different experiences of stigma impact psychological well-being differently depending on the setting in which the stigma occurs has both theoretical and practical implications. Firstly, it suggests that setting- and manifestation-specific measures of HIVrelated stigma likely provide insight that aggregate measures cannot. Secondly, it points to the importance of gearing stigma reduction interventions to specific manifestations in specific settings. In order to do this effectively, additional research on family and health 
care settings is necessary (see Bos, Schaalma, \& Pryor, 2008; L. Brown, Macintyre, \& Trujillo, 2003)).

Some limitations to this study should be mentioned. First, compared to the general Dutch PLWH population (HIV Monitoring Foundation, 2008), our study overrepresented gay men, people with a high level of education, and people with a Western background. Although correlational analyses showed no associations between these variables and psychological distress, caution should be applied when generalizing findings. A second limitation is the response rate. We endeavored to increase response rates via personal contact and followup reminders, and succeeded in reaching $6 \%$ of all diagnosed PLWH in the Netherlands. Nonetheless, the potential for non-response bias cannot be dismissed. A third limitation is the cultural setting in which the survey occurred. The Netherlands is a fairly tolerant culture. As such, it is possible that Dutch PLWH experience less stigmatization and psychological damage than PLWH elsewhere. Although our findings support research on HIV-related stigma and psychological distress conducted in other countries (Clark, Lindner, Armistead, \& Austin, 2003; Kang, Rapkin, Remien, Mellins, \& Oh, 2005; R. S. Lee, Kochman, \& Sikkema, 2002; Mak et al., 2007; Riggs, Vosvick, \& Stallings, 2007; Simbayi et al., 2007; Vanable, Carey, Blair, \& Littlewood, 2006; Wu et al., 2008), we nonetheless recommend replicating our findings in other cultural contexts. A fourth limitation is that this study did not consider the serostatus of interaction partners in settings (e.g. partner, family, friends). We suggest that future research control for this. A final limitation is the cross-sectional study design. While we presumed that stigma impacts psychological well-being, one could contend that the direction of the relationship is the opposite. This, however, would require relatively similar bivariate correlations between psychological distress and most of the manifestations of HIV-related stigma in most of the settings. Instead, we found psychological distress to be most strongly associated with very specific manifestations in specific settings. Consequently, we contend that the direction of the relationship assumed is more likely than its alternative.

In conclusion, this study has uniquely contributed to our understanding of the relationship between HIV-related stigma and psychological distress, and added to previous research by demonstrating that it is possible to identify the specific manifestations occurring in specific social settings that are most detrimental to psychological well-being. 
HIV-RELATED STIGMA AND PSYCHOLOGICAL DISTRESS 


\section{PSYCHOLOGICAL AND SOCIAL CORRELATES OF HIV STATUS DISCLOSURE: THE SIGNIFICANCE OF STIGMA VISIBILITY}

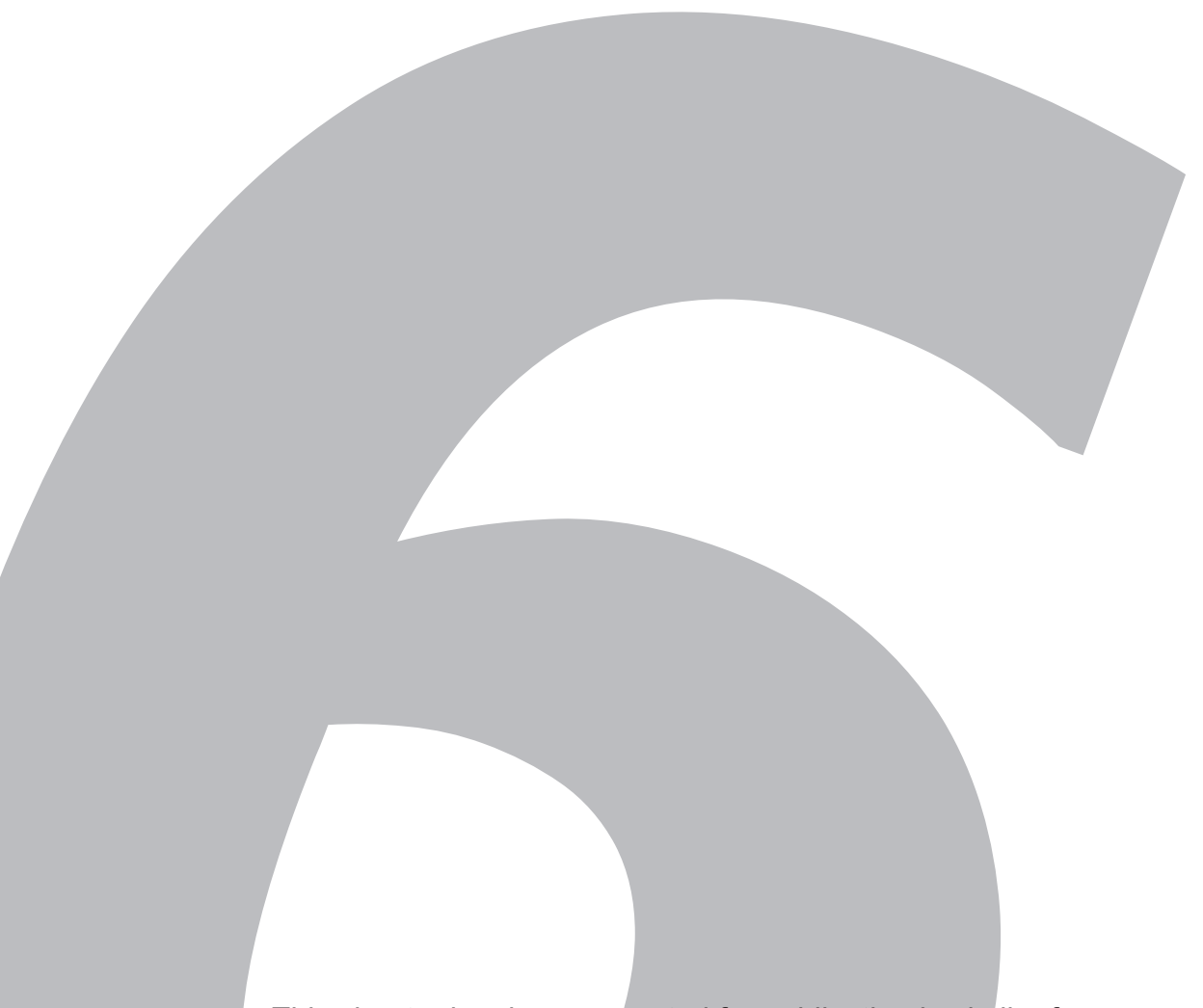

This chapter has been accepted for publication in similar form as:

Stutterheim, S. E., Bos, A. E. R., Pryor, J. P., Brands, R., Liebregts, M., \& Schaalma, H. P. (in press). Psychological and social correlates of HIV status disclosure: The significance of stigma visibility, AIDS Education and Prevention, 23(3). 


\section{ABSTRACT}

HIV-related stigma, psychological distress, self esteem, and social support were investigated in a sample comprising people who have concealed their HIV status to all but a selected few (limited disclosers), people who can conceal but chose to be open (full disclosers), and people who had visible symptoms that made concealing difficult (visibly stigmatized). The visibly stigmatized and full disclosers reported significantly more stigma experiences than limited disclosers, but only the visibly stigmatized reported more psychological distress, lower self esteem, and less social support than limited disclosers. This suggests that having a visible stigma is more detrimental than having a concealable stigma. Differences in psychological distress and self esteem between the visibly stigmatized and full disclosers were mediated by social support while differences between the visibly stigmatized and limited disclosers were mediated by both social support and stigma. These findings suggest that social support buffers psychological distress in people with HIV. 


\section{INTRODUCTION}

A stigma is a distinctive, discrediting characteristic that renders its bearer tainted, flawed, or inferior in the eyes of others (Bos, Kok, \& Dijker, 2001; Crocker, Major, \& Steele, 1998; Goffman, 1963; Jones et al., 1984; Major \& O'Brien, 2005). A fundamental dimension of stigmas concerns the degree to which they can be concealed from others. People who choose to 'pass' as 'normal' by concealing their stigma nevertheless remain 'discreditable' as long as there is a potential that the stigma can be revealed (Goffman, 1963). Concerns regarding who to tell and the fear of being discovered are significant sources of psychological distress among those who conceal stigmas (Pachankis, 2007). Those who voluntarily disclose their stigmatized status or those who have conspicuous stigmas must endure potentially being 'discredited' in the eyes of others. People living with HIV (PLWH) run the gamut with regard to these three different varieties of stigma experience. Some try to pass, telling virtually no one or only a selected few. Others choose to openly reveal their status. Still others have conspicuous symptoms that make passing difficult. In the current study, we explored the psychological and social consequences of these three different kinds of disclosure choices.

The current literature indicates that both disclosure and concealment have positive and negative consequences. Numerous studies have documented negative reactions to HIV status disclosure (Alonzo \& Reynolds, 1995; Black \& Miles, 2002), and the subsequent detrimental consequences for psychological well-being (Bing et al., 2001; Heckman et al., 2004; Pence, Miller, Whetten, Eron, \& Gaynes, 2006; Stutterheim et al., 2009) and social relationships (J. D. Lee \& Craft, 2002) thus suggesting that it would be wise to keep one's HIV status a secret. Others have shown that concealing a stigmatized condition also has very substantial psychological and social costs, including stress (Greenberg \& Stone, 1992; Pennebaker, Colder, \& Sharp, 1990), poor mental health outcomes (Derlega, Winstead, Oldfield, \& Barbee, 2003; Steward et al., 2008; Ullrich, Lutgendorf, \& Stapleton, 2003), strained social interactions (Smart \& Wegner, 1999), social isolation (Corrigan \& Matthews, 2003; Remennick, 2000), and the insufficient provision of social support (Alonzo \& Reynolds, 1995; Chesney \& Smith, 1999). The role of social support is particularly important as it not only enables PLWH to better cope with health concerns (Smith, Rossetto, \& Peterson, 2008) but also buffers stress, anxiety, and depression that can result from, among other things, stigmatization. (Kalichman, DiMarco, Austin, Luke, \& DiFonzo, 2003; Lam, Naar-King, \& Wright, 2007; Li, Lee, Thammawijaya, Jiraphongsa, \& Rotheram-Borus, 2009). However, a prerequisite for the receipt of social support is 
precisely that which can generate stigmatization: disclosure. In essence, PLWH must take the risk of being met with stigmatizing reactions in order to gain the support necessary to deal with stigmatizing reactions. Evidently, PLWH who are in a position to conceal their status are faced with difficult decisions regarding whether or not they should disclose or conceal.

For some PLWH, disclosure is involuntary. Disease progression and, more frequently, side effects of highly active antiretroviral therapy (HAART), such as lipodystrophy syndrome, can make HIV a condition with conspicuous symptoms. The psychological and social implications of HIV may vary according to the presence or absence of visible symptoms. PLWH with visible symptoms may, in fact, be better off than PLWH who can conceal their condition. Research conducted by Frable, Platt, and Hoey (1998) compared concealable and visible stigmas and found that those with concealable stigmas (i.e. sexual orientation, bulimia, or very low socioeconomic status) had more anxiety, depression, and negative affect, as well as lower self esteem, than those with visible stigmas (i.e. ethnicity or overweight). This would suggest that people with visible stigmas fare better than those who try to conceal, perhaps because they have access to an array of possible coping strategies that might not be readily available to those whose stigma is hidden (Quinn, 2006). For example, people with visible stigmas are often in a better position to find and compare themselves to in-group members, and they might more readily attribute negative treatment to prejudice (Crocker, Major, \& Steele, 1998). To our knowledge, no prior studies have examined how the presence of visible symptoms impacts the stigma experiences of people with HIV.

In the current study, we examined HIV-related stigma, psychological distress, self-esteem, and social support in a sample of people known to have HIV. Our participants fit into three categories, namely people who have concealed their HIV status to all but a selected few (limited disclosers), people who are able to conceal their status but chose to be open about it to others (full disclosers), and people who felt they had visible symptoms that make their status difficult to conceal to others (visibly stigmatized). One of the unique features of this study is that we were able to compare the consequences of stigma visibility to those of stigma concealment or disclosure across groups that had essentially the same stigma. Although one might argue that having visible symptoms represents a qualitatively different stigma, some important factors, such as the stereotypes about PLWH and the degree to which PLWH are blamed for their condition, are constant across these different experiences of stigma. 


\section{METHODS}

\section{Participants and procedure}

Data were obtained via an anonymous survey for which participants provided informed consent. Participation was voluntary and did not involve monetary compensation. Approval from the Ethics Committee at Maastricht University's Faculty of Psychology and Neuroscience was provided. In total, 2,264 surveys were distributed to PLWH in 2007 by the Dutch HIV Association and by HIV nurses. Of the 2,264, 669 surveys were returned (response rate=29.5\%). Two surveys were excluded from the analyses as the corresponding participants were outliers with respect to age (6 and 97 years).

Of the 667 participants included, $86.2 \%$ were male and $13.8 \%$ were female. Ages ranged from 17 to 75 with a mean age of $46.6(S D=9.6)$. Almost half of the respondents $(49.5 \%)$ had at least a Bachelor's degree. An additional $31.0 \%$ had a high school diploma and/or some vocational training, and $19.5 \%$ had a high school diploma or less. Further, $68.3 \%$ of participants had paid employment and $48.4 \%$ of participants had a long term partner. The greater majority of the sample defined themselves as gay $(79.5 \%)$ and from Europe or North America (90.6\%). Most of the participants (87.5\%) had acquired HIV through sexual intercourse and the mean time since diagnosis was 8.75 years $(S D=6.0)$. A total of $79.3 \%$ of participants were being treated with antiretroviral therapy at the time of the study.

\section{Measures}

Disclosure of HIV status was measured using questions that addressed disclosure to several potential targets ("Who have you told that you have HIV?"). For their long term partner, mother, and father, participants answered 'yes', 'no', or 'not applicable'. With respect to disclosure to immediate and extended family members (excluding mother and father), friends, acquaintances, and colleagues, answers were provided on a five-point scale ranging from 1 ([almost] no one) to 5 ([almost] everyone).

HIV-related stigma experiences were assessed using a 15-item scale developed by the authors. Participants indicated the degree to which they had experienced negative reactions to their HIV status in a number of social settings ("To what extent have you experienced negative reactions to your HIV status in each of the following situations?"; examples of settings: family, friends, other PLWH, work, health care sector, faith community, gay community) on a five-point scale ranging from 1 (never) to 5 (very often). A higher score is indicative of greater stigma. Cronbach's alpha is .77. 
Psychological distress was measured using the 18-item Mental Health Inventory (MHI) which measures depression, anxiety, positive affect, and behavioral control (Veit \& Ware, 1983). Answers were provided on a six-point scale ranging from 1 (none of the time) to 6 (all of the time). A higher score is indicative of more psychological distress. This scale has been used extensively and is considered to be both valid and reliable (Rosenthal, Downs, Arheart, \& Deal, 1991; Veit \& Ware, 1983). Cronbach's alpha is .94. An example of an item is: "How much of the time, during the past four weeks, have you felt downhearted and blue?"

Self esteem was assessed using the Rosenberg Self Esteem Scale (RSE; Rosenberg, 1965) which contains ten items, all of which are scored on a four-point scale ranging from 1 (strongly disagree) to 4 (strongly agree). A higher score is indicative of greater self esteem. The RSE is a frequently used measure of self esteem (Schmitt \& Allik, 2005). Cronbach's alpha is .88. An example item is: "I am able to do things as well as most other people."

Social support was measured using the 12-item short version of the Social Support List of Interactions (SSL-12) which measures the frequency of everyday support, social support in problem situations, and esteem support (Kempen \& Van Eijk, 1995). Answers were provided on a four-point scale ranging from 1 (seldom or never) to 4 (very often). A higher score is indicative of more social support. This scale has good psychometric properties (Kempen \& Van Eijk, 1995). Cronbach's alpha is .73. An example item is "Does it ever happen to you that people drop in for a visit?"

The presence of visible symptoms was measured by one item, namely, "Do you currently have visible symptoms as a result of your HIV infection?," to which participants responded with 'yes' or 'no'. Those that responded affirmatively were subsequently asked to describe those symptoms. Responses included lipodystrophy syndrome, dermatological complaints, and neurological symptoms.

Demographic characteristics and HIV-related characteristics were also measured. HIV-related characteristics included the mode by which one acquired HIV, the time since diagnosis, current treatment with HAART, and self-reported current health status. Demographic characteristics measured included gender, age, educational attainment, current employment, marital status, sexual orientation, and ethnic background.

\section{RESULTS}

Descriptive statistics showed that $97.9 \%$ of participants had disclosed their HIV status to their long term partner, $68.2 \%$ to their mother, and $64.7 \%$ to their father. Also, $65.0 \%$ 
reported having disclosed to most other family members and $64.1 \%$ to most friends. Disclosure rates to acquaintances and colleagues were lower with $31.1 \%$ having disclosed to most acquaintances and $28.8 \%$ to most colleagues.

As stated above, groups were distinguished according to their disclosure status (full disclosers, limited disclosers, and the visibly stigmatized). Full disclosers were those participants that had disclosed to their partner and most of their family, friends, acquaintances, and colleagues in the absence of visible symptoms $(N=300)$. Limited disclosers were those participants that did not have visible symptoms and that had opted not to disclose their status in more public settings (i.e. to colleagues and acquaintances; $N=163$ ). The visibly stigmatized were those participants that reported visible symptoms and disclosure to most disclosure targets $(N=194) .{ }^{1}$ An additional group of nondisclosers comprised participants that had told no one $(N=10)$ but was not included in the analyses because of its size.

Means, standard deviations, and inter-correlations for HIV-related stigma, psychological distress, self esteem, and social support are displayed in Table 6.1. One-way analyses of variance-least significant differences (ANOVA-LSD; see Table 6.2) showed that full disclosers and the visibly stigmatized reported significantly more stigma experiences than limited disclosers, $F(2,654)=27.08, p<.001$. They also demonstrated that visibly stigmatized participants reported significantly more psychological distress, $F(2,637)=$ $4.43, p<.05$, lower self esteem, $F(2,637)=4.62, p<.01$, and less social support, $F(2,633)=$ $8.68, p<.001$, than limited disclosers or full disclosers. Limited and full disclosers, in turn, did not differ significantly from one another on psychological distress, self esteem, and social support.

\footnotetext{
${ }^{1}$ Given the nature of their disclosure, these groups differ with respect to certain demographic and HIV-related characteristics. Significant differences were found with respect to age (the visibly stigmatized were older than full disclosers who, in turn, were significantly older than limited disclosers), ethnicity (non-Western ethnicity was more common among limited disclosers), children (limited disclosers had more children), employment (paid employment was lower among the visibly stigmatized), sexual orientation (full disclosers were more likely to be gay), health (poorer health was reported among the visibly stigmatized), time since diagnosis (the visibly stigmatized knew about their HIV status longer than full disclosers who, in turn, have known their status longer than limited disclosers), antiretroviral therapy (the visibly stigmatized were more likely to be receiving therapy), and time since therapy was initiated (time was longest among the visibly stigmatized followed by the full disclosers and then the limited disclosers). No significant differences were found for gender, marital status, or educational attainment.
} 
Table 6.1: Means, standard deviations, and inter-correlations of main variables

\begin{tabular}{lccccc}
\hline & Mean & $S D$ & 1 & 2 & 3 \\
\hline 1. HIV-related stigma & 4.04 & 3.27 & - & & \\
2. Psychological distress & 2.73 & 0.88 & $.24^{* * *}$ & - & \\
3. Self esteem & 3.07 & 0.50 & $-.17^{* * *}$ & $-.73^{* * *}$ & - \\
4. Social support & 2.71 & 0.43 & $-.11^{* *}$ & $-.19^{* * *}$ & $.27^{* * *}$ \\
\hline
\end{tabular}

${ }^{* *} p<.01,{ }^{* * *} p<.001$

Table 6.2: Group comparisons of limited disclosers, full disclosers, and the visibly stigmatized on main variables

\begin{tabular}{crrr}
\hline & Limited disclosers & Full disclosers & Visibly stigmatized \\
\hline HIV-related stigma & $2.50^{\mathrm{a}}$ & $4.39^{\mathrm{b}}$ & $4.78^{\mathrm{b}}$ \\
Mean & 2.84 & 3.12 & 3.37 \\
$S D$ & 163 & 300 & 194 \\
$N$ & & & $2.88^{\mathrm{b}}$ \\
Psychological distress & $2.66^{\mathrm{a}}$ & $2.65^{\mathrm{a}}$ & .88 \\
Mean & .86 & .86 & 190 \\
SD & 158 & 292 & $2.99^{\mathrm{b}}$ \\
$N$ & & & .50 \\
Self esteem & $3.12^{\mathrm{a}}$ & $3.12^{\mathrm{a}}$ & 190 \\
Mean & .54 & .47 & \\
SD & 159 & 291 & $2.61^{\mathrm{b}}$ \\
$N$ & & & .45 \\
Social support & $2.74^{\mathrm{a}}$ & $2.77^{\mathrm{a}}$ & 186 \\
Mean & .46 & .38 & 293 \\
SD & 157 & & \\
$N$ & & &
\end{tabular}

Means in a given row that do not share a common superscript differ at the .05 level

In order to better understand why significant differences were found between the visibly stigmatized and full disclosers on psychological distress, self esteem, and social support despite similar levels of exposure to stigmatization, we conducted mediation analyses according to the method outlined by Baron and Kenny (1986). In brief, this method comprises a series of regression analyses. First, the dependent variable is regressed on the independent variable (Step 1); then the potential mediator is regressed on the 
independent variable (Step 2); subsequently, the dependent variable is regressed on the potential mediator (Step 3); and, lastly, the dependent variable is regressed on both the independent variable and the potential mediator (Step 4). Mediation is satisfied if the independent variable affects both the dependent variable (Step 1) and the mediator (Step 2 ), the mediator affects the dependent variable in the predicted direction (Step 3), and the effect of the independent variable is less significant in Step 4 than in Step 1. Our mediation analyses (see Figure 6.1) demonstrated that differences in psychological distress and self esteem between the visibly stigmatized and the full disclosers were fully mediated by social support, Sobel's $z=-2.91, p<.01$ (see Figure 6.1A) and Sobel's $z=3.49, p<.001$ (see Figure $6.1 \mathrm{~B})$, respectively. Subsequent analyses also showed that differences in psychological distress and self esteem between the visibly stigmatized and limited disclosers were fully mediated by not only social support, Sobel's $z=-2.70, p<.01$ (see Figure $6.1 \mathrm{C}$ ) and Sobel's $z=2.39, p<.05$ (see Figure 6.1D), but also by stigma experiences, Sobel's $z=-3.76, p<.001$ (see Figure 6.1E) and Sobel's $z=2.12, p<.05$ (see Figure 6.1F).

\section{DISCUSSION}

This study is, to our knowledge, the first to explore the psychological and social correlates of full and limited disclosure of HIV status in the presence and absence of visible symptoms. In our comparison of limited disclosers, full disclosers, and visibly stigmatized PLWH, we found that participants with visible symptoms of HIV were at the greatest disadvantage, both psychologically and in terms of social support. These participants reported substantially more psychological distress, lower self esteem, and less social support than participants that were in a position to conceal their status, be they full or limited disclosers. This suggests that having a visible stigma is more detrimental than having a concealable stigma.

One could argue that the reason why visibly stigmatized participants report poorer psychological and social well-being is because they experience more stigma than participants who can conceal. Our results, however, do not support this contention. In fact, in our study, the visibly stigmatized and the full disclosers did not differ from one another in terms of the amount or frequency of stigma experiences. They did, however, vary significantly in their mental health outcomes. Our mediation analyses suggest that this is attributable to social support. Social support was found to mediate the differences in psychological distress and self esteem between the visibly stigmatized participants and the full disclosers thus suggesting that social support may be an important buffer against the negative psychological consequences of stigmatization.

When we explored the differences in psychological distress and self esteem between limited disclosers and the visibly stigmatized, we found that both stigma experiences and 


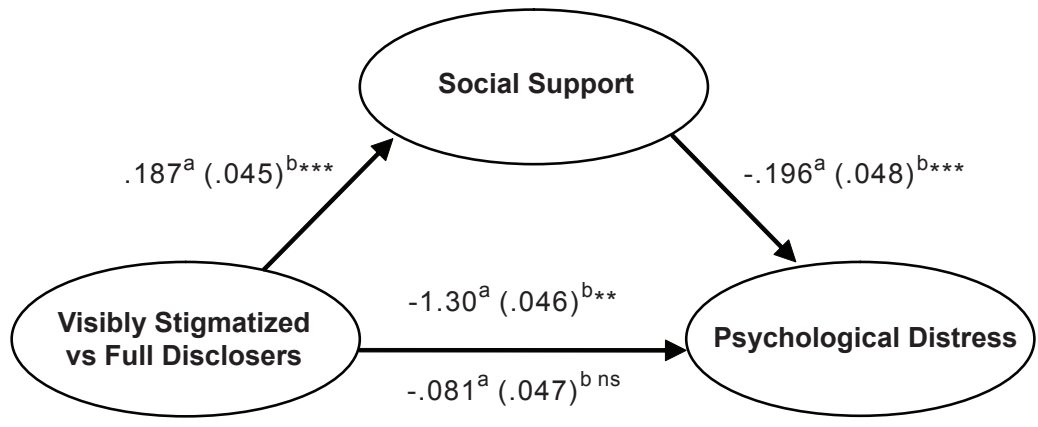

Figure 6.1A

$z=-2.91^{\text {** }}$

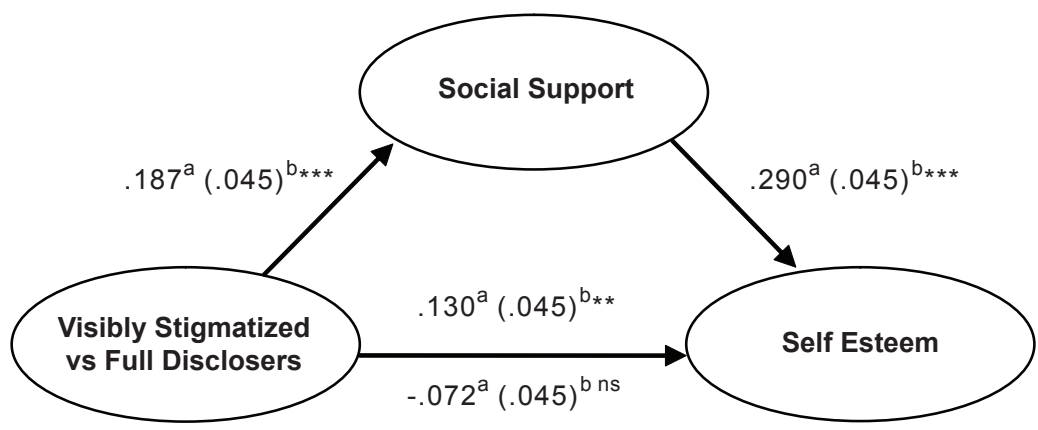

Figure 6.1B

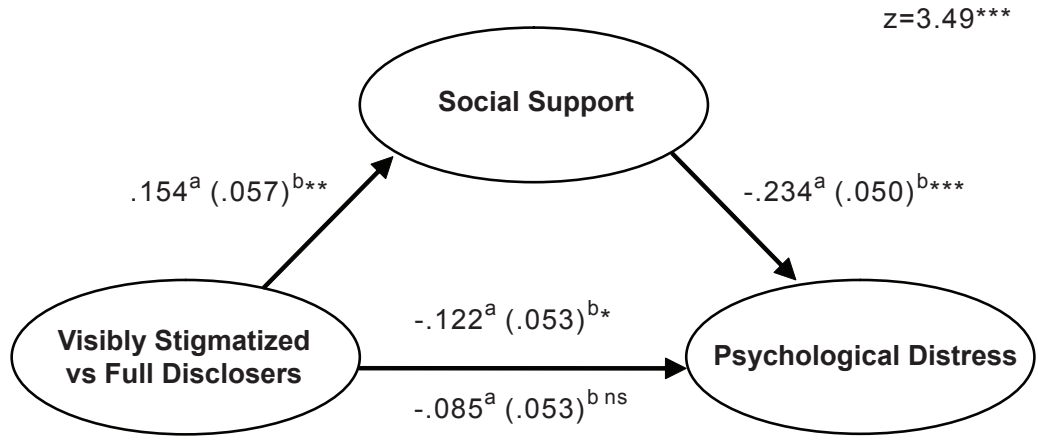

Figure 6.1C

$z=-2.70^{* * *}$

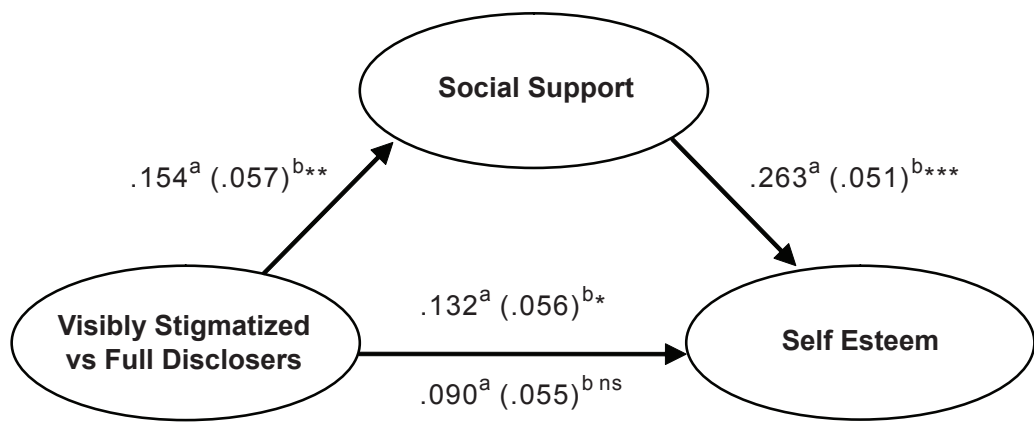

Figure 6.1D 


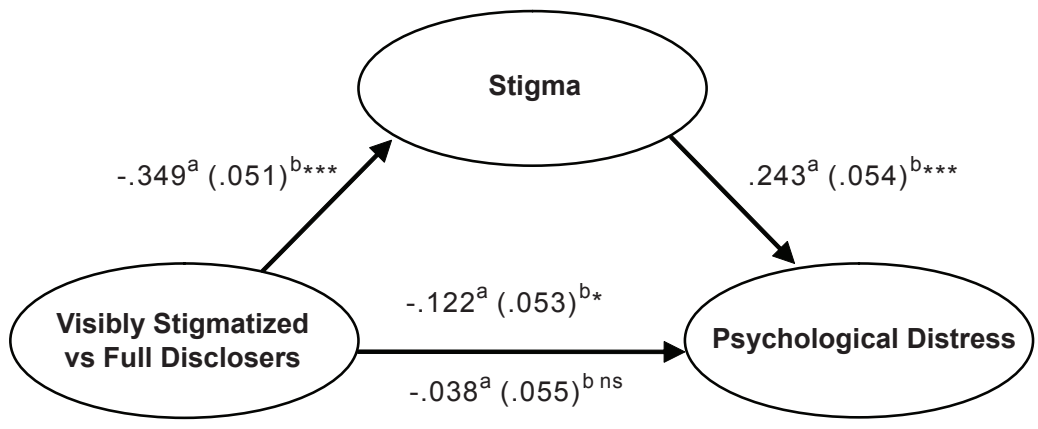

Figure 6.1E

$z=-3.76^{* * *}$

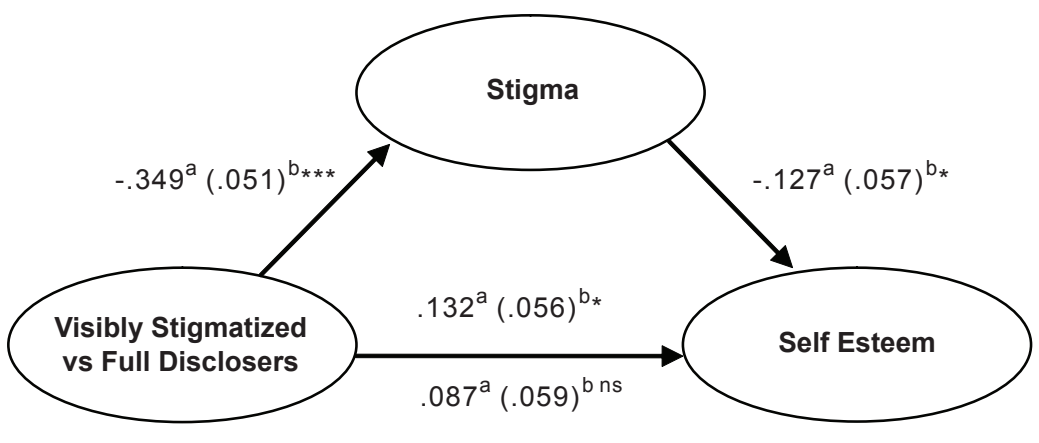

Figure 6.1F

$z=2.12^{*}$

\section{Figure 6.1: Mediation analyses of differences in psychological distress and self esteem between groups}

$\mathrm{a}=$ unstandardized regression coefficient; $\mathrm{b}=$ standard error

${ }^{*}=p<.05 ;{ }^{* *}=p<.01 ;{ }^{* * *}=p<.001$

In accordance with Baron and Kenny (1986), Step 1: text above the horizontal line; Step 2: text to the left of the diagram; Step 3: text to the right of the diagram; Step 4: text under the horizontal line.

social support mediated these differences thus suggesting that limited disclosers have less psychological distress not only because they experience less stigma but also because they receive the necessary support to buffer the stigma they do experience.

Clearly, social support can be a buffer against psychological distress in PLWH. In our study, PLWH with visible symptoms reported significantly less social support than their concealable counterparts. This may be attributable to the nature of their HIV status disclosure. Previous research has shown that the way in which disclosure occurs can impact disclosure targets' responses (Bos, Dijker, \& Koomen, 2007). The selection of the optimal setting, person, and time may thus enable more positive reactions to HIV status disclosure. Unfortunately, people with visible symptoms like lipodystrophy syndrome are less able to determine the conditions under which they disclose as they are often 'outed' by 
their looks. As such, their disclosure is less likely to be voluntary (Joachim \& Acorn, 2000). This is in line with research by Buzzella, Beals, and Peplau (2003) who, in their study on the disclosure of sexual orientation, found that involuntary disclosure is significantly related to less social support. It may also be that voluntary disclosers actually disclose for the purposes of gaining support and thus receive more social support upon disclosure than those who are subjected to involuntary disclosure.

Our finding that PLWH with visible symptoms experienced more psychological distress and lower self esteem corresponds with work conducted by Reynolds and colleagues (2006) and by Sanches et al. (2009), both of whom have demonstrated a relationship between psychological distress and visible HIV symptoms. However, it does not correspond with the work of Frable and colleagues (1998) who have shown that people with a concealable stigma are at greater psychological disadvantage than people with a visible stigma. The incongruence between Frable and colleagues (1998) findings and ours may be the result of the fact that Frable and colleagues compared groups with fundamentally different stigmatized identities (sexual orientation, bulimia, and very low socioeconomic status versus ethnicity and overweight) while we compared three groups that all share the same stigmatized condition, namely HIV. In other words, factors that differentiated the stigmas studied by Frable and her colleagues other than concealability might have contributed to the psychological differences they found across stigmatized groups.

In our comparison of limited disclosers and full disclosers, we found that the only significant difference between the two groups was that the full disclosers had been exposed to more stigma experiences. This finding is in line with research by Bos and colleagues (2009) who previously found that selective disclosure limits stigmatizing responses to mental illness disclosure. The fact that no differences were found in psychological distress, self esteem, and social support, despite significant differences in stigma experiences, is noteworthy. Perhaps full disclosers possess certain attributes (e.g. self-efficacy, self-confidence) and coping mechanisms (e.g. a greater tendency to attribute externally) to a greater extent than limited disclosers. This corresponds with the work of Paxton (2002b) who has shown that public disclosure can lead to psychological release. We recommend that future studies explore such attributes and coping mechanisms as possible mediators or moderators of the relationship between stigma experiences and psychological distress.

There are limitations to the study presented here. First, our study was conducted with a sample of predominantly gay men with a relatively high level of educational attainment and a European or North American background. This may impact the generalizability of our findings. Future research should endeavor to oversample ethnic minorities, heterosexual women, and people with a lower level of education. A second limitation is the cross- 
sectional study design and the resulting difficulties determining causality. We suggest that future studies adopt a longitudinal design. A third limitation is the response rate. We sought to increase response rates via personal contact and follow-up reminders, and were successful in reaching $6 \%$ of all diagnosed PLWH in the Netherlands. However, the potential for non-response bias cannot be dismissed. Further, we acknowledge that our measurement of visible symptoms is self-reported and thereby impacted by participants' perceptions of what is visible and what is not. Future research may benefit from using a more objective measure of visible symptoms (e.g. medical diagnoses of lipodystrophy syndrome). A final limitation is that a group of nondisclosers was not included in this study. Although nondisclosers are hard to find and include in these kinds of studies, as was the case with our study, including such a group may shed greater light on the psychological and social impact of disclosure versus concealment. Future studies should seek to include such individuals despite the difficulties involved in their recruitment.

This study has a number of theoretical and practical implications. In terms of theory, the findings contribute to the debate on whether it is more advantageous to have a visible or concealable stigma (Frable, Platt, \& Hoey, 1998; Pachankis, 2007; Quinn, 2006). Our findings clearly support the contention that visible stigmas are psychologically and socially more detrimental than concealable ones. Our findings also contribute to the ongoing discussion regarding whether concealment or disclosure is better among those that are in a position to conceal their stigmatized identity. Our findings have shown that, although full disclosers experience more stigma than limited disclosers, they do not experience more or less psychological distress or social support. This suggests that future work on the psychological impact of concealment or disclosure should go beyond a dichotomous distinction between disclosers and nondisclosers and explore the impact of varying degrees of disclosure.

In terms of practice, the finding that social support plays an important protective role in the preservation of PLWH's psychological well-being and self esteem is highly relevant. It points to the need to promote social support provision to $\mathrm{PLWH}$, especially those with visible symptoms. Health care providers should endeavor to provide such support via their own personal contact with PLWH and also by referring PLWH to important support groups. Theory and evidence-based efforts and interventions to positively connect PLWH with their families and friends in ways that promote social support provision and reduce negative reactions to HIV status disclosure are also advised. Such interventions can focus on empowering PLWH, developing disclosure skills in PLWH, and providing information to disclosure targets that is likely to reduce negative responses (e.g. information indicating that HIV cannot be spread through casual social contact, information showing that PLWH 
can live long and healthy lives with HAART). For additional recommendations on how to reduce negative reactions to HIV status disclosure, see Bos, Pryor, and Schaalma (2008) and Brown, Macintyre, and Trujillo (2003). Clearly, the creation of supportive environments for PLWH and the development of HIV-related stigma reduction interventions are imperative to the promotion of positive HIV status disclosure experiences. 
THE SIGNIFICANCE OF STIGMA VISIBILITY 


\section{GENERAL DISCUSSION}


Since the early years of the epidemic, HIVIAIDS has impacted not only the physical health of people living with HIV (PLWH) but also their psychological well-being and social lives. Much of this is attributable to HIV-related stigma. In this dissertation, social and psychological processes contributing to, and resulting from, HIV-related stigma have been investigated. The studies included in this dissertation have explored the beliefs underlying HIV-related stigma, the manifestations of this stigma, the consequences of stigma for PLWH's well-being, the coping mechanisms employed by PLWH to deal with HIV-related stigma, and issues pertaining to disclosure of HIV status. The first part of this dissertation reported on qualitative research conducted with populations disproportionately affected by the HIV epidemic, namely African and Afro-Caribbean diaspora communities in the Netherlands. The second part of this dissertation investigated some aspects of HIV-related stigma in more detail, and with a broader study population. In particular, it explored the social and psychological implications of different manifestations of HIV-related stigma, and of different forms of disclosure. The findings of these studies are summarized and discussed in the context of the existing literature in the sections below.

\section{BELIEFS CONTRIBUTING HIV-RELATED STIGMA}

Chapter 2 explored which beliefs held by African, Antillean, and Surinamese diaspora contribute to the stigmatization of PLWH in their communities and the cultural context of these beliefs. Through interviews with both HIV-positive and HIV-negative community members, we established that a number of beliefs previously found to contribute to HIVrelated stigma in communities indigenous to developed countries (Bos, Dijker, \& Koomen, 2007; Dijker \& Koomen, 2003; Herek, 1999) are also held by members of these diaspora communities.

The first is the belief that HIV is highly contagious. In these communities, this belief and the subsequent inclination to be 'careful' around PLWH were found to prevail despite adequate knowledge regarding HIV transmission and prevention. This discrepancy has previously been found in African studies (Maman et al., 2009; Ogden \& Nyblade, 2005), and studies conducted in developed countries have also found that people have aversions to casual contact with PLWH despite apparent awareness of how HIV is actually transmitted (Pryor, Reeder, \& Landau, 1999; Rozin, Markwith, \& Nemeroff, 1992).

The second belief is that HIV is very severe. In these communities, HIV was associated with death and wasting. Similar associations have been found in studies conducted in Africa (de-Graft Aikins, 2006; Nyblade, Pande, Mathur, MacQuarrie, \& Kidd, 2003; Visser, Makin, \& Lehobye, 2006), the Caribbean (Varas-Diaz, Serrano-Garcia, \& Toro-Alfonso, 2005), 
and among African and Caribbean diaspora (Burns, Imrie, Nazroo, Johnson, \& Fenton, 2007; Dodds et al., 2004; Gardezi et al., 2008). The association between HIV and death is unsurprising given that the participants originate from countries where highly active antiretroviral therapy (HAART) has, until recently, been or currently remains unavailable or difficult to access.

The third belief is the belief that PLWH are personally responsible for their HIV infection. In these communities, HIV is often viewed as punishment or due justice for poor or immoral behavior, thus generating less compassion for HIV than for other medical conditions. Attributions of personal responsibility have frequently been reported in other studies conducted in Africa (Duffy, 2005; Hartwig, Kissioki, \& Hartwig, 2006; Roura et al., 2008), the Caribbean (Carr, 2004), and in their respective diaspora communities (M. Anderson et al., 2008; Burns, Imrie, Nazroo, Johnson, \& Fenton, 2007; Dodds et al., 2004; Gardezi et al., 2008). In one South African study, $61 \%$ of the participants considered PLWH to be of poor moral character, $22 \%$ considered HIV to be punishment for bad behavior, and 26\% blamed PLWH for contracting HIV (Visser, Makin, \& Lehobye, 2006). The negative relationship between attributions of personal responsibility and compassion for PLWH has also previously been documented (Bos, Kok, \& Dijker, 2001).

The fourth belief contributing to HIV-related stigma in African and Afro-Caribbean diaspora communities is the belief that PLWH acquired HIV because they engaged in behaviors considered norm-violating. Examples of such behaviors are promiscuity, commercial sex work, and homosexuality. Differences in which behaviors were associated with HIV appeared to vary between the communities. For example, the association with homosexuality was present in Afro-Caribbean communities but not in African communities, and the association with commercial sex work was stronger in African communities than in Afro-Caribbean communities. These differences are likely due to differences in the HIV epidemics in participants' home countries. The link between HIV and promiscuity and commercial sex has previously been established in studies conducted in a number of Sub-Saharan African countries (Duffy, 2005; Nyblade, Pande, Mathur, MacQuarrie, \& Kidd, 2003), in the Caribbean (White \& Carr, 2005), and among diaspora of African and Caribbean descent (M. Anderson et al., 2008; Dodds et al., 2004). The association between HIV and homosexuality has also been established in studies with Caribbean people, both in their home countries (Carr, 2004; White \& Carr, 2005) and in their diaspora communities (M. Anderson et al., 2008; Gardezi et al., 2008).

An additional finding of this study on the beliefs contributing to HIV-related stigma was that taboos on HIV and sexuality exacerbate the beliefs that contribute to HIV-related stigma. 
This contributing role of taboo to HIV-related stigma has previously been noted by studies conducted in Sub-Saharan Africa (Campbell, Foulis, Maimane, \& Sibiya, 2005; Roura et al., 2008) and in studies with African and Caribbean diaspora (Burns, Imrie, Nazroo, Johnson, \& Fenton, 2007; Dodds, 2006; Gardezi et al., 2008). Taboos can function to perpetuate myths and reinforce attributions of blame and norm violation by impeding knowledge acquisition and increased familiarity with HIV and PLWH, both of which have been found to enable the reduction of HIV-related stigma.

\section{SETTINGS AND MANIFESTATIONS OF HIV STIGMA}

In Chapter 3, the manifestations of stigma in African and Afro-Caribbean communities were qualitatively explored as comprehensive knowledge regarding how stigma manifests in African and Afro-Caribbean diaspora communities is limited. Drawing on the perspectives of both HIV-negative (perceivers) and HIV-positive community members (targets), we established that HIV-related stigma manifests in these communities as social distance, physical distance, words, and silence. Social distance includes avoidance, rejection, abandonment, and social exclusion, and was found to occur across a number of social settings including families, friends, and romantic partners. Physical distance as a manifestation of HIV-related stigma was reported to pertain not only to PLWH but also to objects PLWH touch such as food, dishes, toilets, and chairs. Stigmatizing words take the form of gossip, violations of confidentiality, blaming, and disdain when HIV status is known or assumed, and as negative remarks regarding HIV and PLWH when HIV status is unknown. Lastly, in these communities, silence was also reported to be a manifestation of HIV-related stigma that occurs particularly in families but also in one's community, and that is considered attributable to familial shame and cultural taboos on talking about HIV. These findings are corroborated by research conducted with African and Caribbean people in diaspora communities (M. Anderson et al., 2008; Dodds et al., 2004; Gardezi et al., 2008; Kinniburgh, Scott, Gottlieb, \& Power, 2001) and in their countries of origin (Bond, Chase, \& Aggleton, 2002; Duffy, 2005; Varas-Diaz, Serrano-Garcia, \& Toro-Alfonso, 2005). For example, in research conducted by Kinniburgh and colleagues (2001), African PLWH in the United Kingdom reported being judged by their families and abandoned by their partner. They also reported a climate of secrecy regarding HIV in their community, as did Gardezi et al. (2008) in their study with African and Afro-Caribbean communities in Canada. Also, in a study conducted by Anderson and colleagues (2008), Jamaicans living in the United Kingdom reported gossip and verbal abuse by members of their community, and excessive protective measures (e.g. disinfecting clothes, forcing PLWH to use separate plates and silverware) by family members. 
In addition to a qualitative account of HIV-related stigma manifestations in African and Afro-Caribbean communities, this dissertation includes quantitative findings on the settings and manifestations in which HIV-related stigma occurs. In Chapter 5, the prevalence of stigma manifestations was investigated using a broader sample of PLWH. About one in every three participating PLWH had experienced blaming, increased physical distance, avoidance, excessive hygienic measures, indifference, and/or exclusion, and one in every twelve had been subjected to some form of aggression as a result of being HIV-positive. The results also showed that while more than half of participants had been advised to conceal their HIV status, more than a quarter had been encouraged to disclose their HIV status to others. Similar prevalence rates for manifestations such as avoidance and exclusion have previously been found in other studies conducted in the United States (Gielen et al., 2000; Swendeman, Rotheram-Borus, Comulada, Weiss, \& Ramos, 2006; Vanable, Carey, Blair, \& Littlewood, 2006).

Chapter 5 also explored differences in the frequency of stigma manifestations across a number of social settings, namely with friends, family, one's partner, the health care sector, at work, and in leisure settings. The findings demonstrated that PLWH are most likely to experience some manifestation of HIV-related stigma in settings with friends, followed by family, people in the health care sector, and people at work. The likelihood of experiencing a manifestation of HIV-related stigma was lowest with one's partner and in leisure activity settings. To our knowledge, differences in frequency had not previously been explored. However, the finding that stigmatization occurs most frequently with friends seems plausible as previous research has shown that PLWH disclose more often to friends than to others (Kalichman, DiMarco, Austin, Luke, \& DiFonzo, 2003; Stempel, Moulton, \& Moss, 1995). Additionally, greater frequency of stigmatization with friends may also, at least in part, be attributable to the fact that one's group of friends may contain a greater absolute number of potentially stigmatizing individuals than other settings. As such, with friends, there are likely more opportunities for stigmatization.

\section{CONSEQUENCES OF HIV RELATED STIGMA}

In addition to exploring the manifestations of HIV-related stigma, both Chapter 3 and Chapter 5 investigated the consequences of HIV-related stigma for PLWH. Chapter 6 also investigated the impact of different forms of HIV status disclosure on psychological and social well-being.

Chapter 3 delineated the psychological, social, and health consequences of stigmatization for African, Dutch Antillean, and Surinamese PLWH. The psychological consequences 
reported were emotional pain, sadness, loneliness, anger, frustration, and internalized stigma. These findings parallel those reported by Nyblade and colleagues (2003) who demonstrated that PLWH in Sub-Saharan Africa experience despondency, despair, and a loss of hope, and internalize stigma. The social consequences reported in Chapter 3 included decreased social network size, limited social support, and social isolation, and were found to result from not only enacted stigma but also self-imposed social withdrawal. This is in line with the work of Smart Richman and Leary (2009) who claimed that chronic and pervasive rejection increases the likelihood of withdrawal and avoidance. Lastly, poor treatment adherence was found to be a health-related consequence of HIV-related stigma. Similar difficulties with treatment adherence among migrant PLWH in the Netherlands have previously been documented by Shiripinda and van Eerdewijk (2008).

Chapter 5 investigated the psychological consequences of HIV-related stigma in more detail by exploring which specific stigma experiences are most strongly related to psychological distress across the previously mentioned social settings (i.e. with friends, with family, with one's partner, in the health care sector, at work, and in leisure settings). The negative psychological impact of 11 manifestations of HIV-related stigma (physical distance, awkward social interaction, indifference, avoidance, blaming, exaggerated kindness, aggression, exclusion, excessive hygienic measures, being told to disclose one's status, and being told to conceal one's status) was first explored according to setting. In the setting friends, blame, awkward social interaction, and exaggerated kindness were found to contribute most to psychological distress. With family, the most detrimental manifestations were being advised to conceal one's status, avoidance, and exaggerated kindness. With one's partner, significant predictors were being told to conceal one's status and exaggerated kindness. In the health care sector, the manifestations predicting psychological distress were indifference and awkward social interaction. No significant predictors were found for the work and leisure settings. All significant setting-specific manifestations were then included in one regression model and four predictors emerged. Three manifestations in family settings, namely receiving advice to conceal one's status, being avoided, and being treated with exaggerated kindness, and one manifestation in health care settings, namely awkward social interaction, best predicted psychological distress in PLWH. Given the literature, it is possible to contend that stigmatization by family may be particularly detrimental because families are not chosen and often considered an important source of unconditional love and support. Stigmatization by family may thus threaten a fundamental human need, namely the need to belong (Baumeister, Leary, Higgins, \& Kruglanski, 2000). With respect to the impact of awkward social interactions with health care professionals, previous research has shown that many PLWH assume that health professionals are knowledgeable about HIV and thus expect them to be at ease 
with them (Green \& Platt, 1997). When health professionals' actions suggest otherwise, disappointment and subsequent psychological distress may ensue. Evidently, this has shown that the psychological impact of HIV-related stigma does vary depending on the manifestation and the setting in which that manifestation occurs. This suggests that future studies measuring the psychological impact of HIV-related stigma could benefit from measures that specify manifestations and settings. To date, most studies investigating the psychological impact of HIV-related stigma employ aggregate measures of stigma and, although these measures do provide useful information regarding the psychological implications of HIV-related stigma in general, they cannot establish differential effects of different manifestations and settings.

Chapter 6 looked at the psychological and social implications of different kinds of disclosure by investigating HIV-related stigma, psychological distress, self esteem, and social support in a sample comprising people who have concealed their HIV status to all but a selected few (limited disclosers), people who can conceal but chose to be open (full disclosers), and people who had visible symptoms that made concealing difficult (visibly stigmatized). In this study, we found that while visibly stigmatized participants and full disclosers both reported significantly more stigma experiences than limited disclosers, only the visibly stigmatized reported more psychological distress, lower self esteem, and less social support than limited disclosers. This suggests that having a visible stigma is more detrimental than having a concealable stigma. This finding corresponds with work conducted by Reynolds and colleagues (2006) and by Sanches et al. (2009), both of whom have demonstrated a relationship between psychological distress and visible HIV symptoms. However, it does not correspond with the work of Frable and colleagues (1998) who have shown that people with a concealable stigma are at greater psychological disadvantage than people with a visible stigma. It is possible that this difference is the result of the fact that Frable and colleagues (1998) compared groups with fundamentally different stigmatized identities (sexual orientation, bulimia, and very low socioeconomic status versus ethnicity and overweight) while we compared three groups that all share the same stigmatized condition, namely HIV. In other words, factors that differentiated the stigmas studied by Frable and colleagues other than concealability might have contributed to the psychological differences they found across stigmatized groups.

An additional finding of the study reported in Chapter 6 was that differences in psychological distress and self esteem between the visibly stigmatized and full disclosers were mediated by social support while differences between the visibly stigmatized and limited disclosers were mediated by both social support and stigma. These findings clearly suggest that social support can buffer psychological distress in PLWH. This is supported by work conducted 
by Lee and Craft (2002) and by Zea and colleagues (2005). The former found that social support aids in coping with the adverse effects of stigmatization while the latter established that social support following HIV status disclosure alleviates depression and enhances self esteem. Also, the finding that limited disclosers experience less stigmatization is in line with research conducted by Bos and colleagues (2009) who found that selective disclosure limits stigmatizing responses to mental illness disclosure. Clearly, the findings suggest that the nature of the stigma (e.g. visible or concealable stigma) and the extent to which disclosure has taken place (e.g. nondisclosure, limited disclosure, or full disclosure) should be considered when studying the psychological impact of different forms of disclosure.

Together, the three studies in Chapters 3, 5, and 6 have demonstrated that HIV-related stigma does indeed have negative implications for PLWH's health and well-being, that some manifestations in certain settings are psychologically more detrimental than others, that certain forms of disclosure are psychologically and socially more detrimental than others, and that social support can buffer the negative psychological impact of HIVrelated stigma.

\section{COPING WITH HIV-RELATED STIGMA}

How PLWH cope with HIV-related stigma was also investigated. In Chapter 3, the coping strategies employed by African and Afro-Caribbean PLWH to mitigate the negative social and psychological consequences of HIV-related stigma were documented as our knowledge regarding which coping strategies are employed by African and Afro-Caribbean diaspora PLWH is still relatively limited. Chapter 3 established that PLWH employ a number of coping strategies that are geared to either altering their relationship with their environment (problem-focused coping) or to regulating negative emotions (emotionfocused coping). Problem-focused coping strategies reported in this study were selectively disclosing or concealing one's HIV status, disengaging or socially withdrawing from stigmatizing individuals, affiliating with similar others, seeking social support from friends and family, and, to a lesser extent, engaging in activism to reduce HIV-related stigma. Emotion-focused coping strategies included distraction or focusing on things or people other than stigmatizing experiences, positive reappraisal of stigmatizing experiences, religious coping, external attributions to ignorance, disidentification with one's stigmatized identity, and acceptance that stigmatization will happen. These findings are line with the theoretical literature on coping with stigma (Crocker, Major, \& Steele, 1998; Miller \& Kaiser, 2001; Miller \& Major, 2000). They also correspond with work conducted with African and Caribbean PLWH. For example, Dodds and colleagues (2004) found that African PLWH 
in the United Kingdom cope by seeking support from other PLWH and their families. In the Caribbean, Carr (2004) found that PLWH cope with stigma by selectively disclosing, seeking social support from friends and family, and by turning to their faith. In Africa, problem-focused (e.g. connecting with other PLWH, seeking support, educating others) and emotion-focused coping responses (e.g. positive thinking, acceptance, religious coping) similar to those found in our study were comprehensively documented by Makoe and colleagues (2008).

\section{DISCLOSURE OF HIV STATUS}

The final aspect of HIV-related stigma investigated in this dissertation was disclosure of HIV status. Previous research has shown that disclosure decisions involve the weighing of costs and benefits (Black \& Miles, 2002; Derlega, Winstead, Greene, Serovich, \& Elwood, 2004; Serovich, 2001; Valle \& Levy, 2009). As such, understanding disclosure patterns requires that we understand the reasons employed by PLWH to justify disclosure or concealment. Consequently, in Chapter 4, the reasons for and against disclosure employed by African and Afro-Caribbean PLWH in the Netherlands were investigated. Reasons for nondisclosure were fear of stigmatization, having had previous negative experiences with disclosure, having observed the stigmatization of other PLWH, feeling ashamed of one's HIV infection, wanting to protect others - particularly one's children and family - from stigmatization-by-association and/or worrying, and believing that one's HIV status is a private matter. With respect to reasons for disclosure, the PLWH in this study reported having or wanting to disclose because they were in a close and supportive relationship, because disclosure can yield emotional release, because disclosure can lead to emotional or financial support, because they feel a perceived duty to inform others, and because they have a desire to educate others about sexual risk-taking. Taken together, the results, and particularly the results pertaining to reasons for nondisclosure, point to the fact that stigma, be it public stigma (discrimination), self stigma (felt and internalized stigma), or stigma-by-association, plays a very important role in disclosure decisions among African and Afro-Caribbean PLWH. This is not surprising given that high levels of stigma have been reported in African and Caribbean countries (M. Anderson et al., 2008; Kalichman \& Simbayi, 2004). In a study conducted with PLWH in Sub-Saharan Africa, Greeff and colleagues (2008) found that refusal to disclose resulted from seeing how other PLWH had been treated, and that the negative effect disclosure could have on the family was a reason for concealment. In a study conducted in Barbados, $30 \%$ of participants indicated fear of stigmatization as the reason for nondisclosure to others and an additional $23 \%$ 
indicated not disclosing to their current sexual partner in order to avoid abnormal reactions and possible violence (Kumar, Waterman, Kumari, \& Carter, 2006).

Additional findings pertaining to disclosure were reported in Chapter 6. This chapter demonstrated how having a choice with respect to disclosure impacts the psychological and social well-being of PLWH. As stated above, the study findings established that PLWH that are 'outed' by their visible symptoms (e.g. lipodystrophy syndrome) are psychologically and socially poorer off than PLWH who are in a position to conceal their HIV status. Previous research has shown that the way in which disclosure occurs can impact disclosure targets' responses and that the selection of an optimal setting, person, and time may enable more positive reactions to the disclosure (Bos, Dijker, \& Koomen, 2007). Unfortunately, when visible symptoms are present, people are less able to determine the conditions under which they disclose. Their disclosure is less likely to be voluntary (Joachim \& Acorn, 2000). This is in line with research by Buzzella, Beals, and Peplau (2003) who, in their study on the disclosure of sexual orientation, found that involuntary disclosure is significantly related to less social support.

\section{HIV-RELATED STIGMA: UNIVERSAL OR CULTURALLY CONSTRUCTED?}

Given that the first part of this dissertation focused on HIV-related stigma in specific cultural contexts, namely African and Afro-Caribbean communities in the Netherlands, the issue of culture and stigma is worthy of discussion. In much of the literature on stigma in general, and HIV-related stigma in particular, claims have been made regarding the impact of culture on the causes, manifestations, and consequences of HIV-related stigma. Many scholars have contended that stigma is socially and culturally constructed (Crocker, Major, \& Steele, 1998; Dovidio, Major, \& Crocker, 2000; Stangor \& Crandall, 2000) and that cultural differences affect what is stigmatized, the extent to which stigmatization occurs, how that stigma is manifested, and how targets of stigmatization are impacted by and cope with stigmatizing experiences (Maman et al., 2009; Norman, Abreu, Candelaria, \& Sala, 2009; Tate, Van Den Berg, Hansen, Kochman, \& Sikkema, 2006; Weiss, Ramakrishna, \& Somma, 2006). Additionally, culture is claimed to impact disclosure processes (Chandra, Deepthivarma, \& Manjula, 2003; Greeff et al., 2008; Simoni et al., 1995; Yoshioka \& Schustack, 2001).

This contention that the various aspects of HIV-related stigma are culturally constructed and thus differ from one culture or community to another seems to demand that all aspects 
of HIV-related stigma be comprehensively explored in each and every community in which stigma is thought to occur. This is a noble but likely infeasible endeavor and, in light of the findings reported in this dissertation, possibly unnecessary. Although the studies in this dissertation sought to illuminate cultural differences in beliefs contributing to HIV-related stigma, the manifestations of this stigma, the consequences for PLWH, how PLWH cope, and how PLWH approach the issue of disclosure, only nuanced differences were found.

With respect to the underlying causes of HIV-related stigma, our findings in Chapter 2 suggest that the beliefs that contribute to HIV-related stigma in communities indigenous to developed countries are also held by African and Afro-Caribbean diaspora communities. When differences between the communities were sought, the only differences found pertained to more detailed aspects of these beliefs. For example, the fear of contagion pertained also to the air among African but not Afro-Caribbean participants, and the association between HIV and homosexuality was made by Afro-Caribbean but not African participants. These differences may be important but they are nuanced. Similarly, the manifestations of stigma reported in Chapter 3 were found to occur across African and Afro-Caribbean communities, and the differences found pertained not to the presence or absence of a given manifestation but rather to the frequency of that manifestation in a community. It is possible that stigmatization occurs more in some cultures than in others. Further, also in Chapter 3, no obvious differences between African and Afro-Caribbean PLWH emerged in terms of the psychological, social, and health consequences of HIVrelated stigma, or in terms of the coping strategies employed to deal with HIV-related stigma. Lastly, in Chapter 4, where reasons for and against disclosure were discussed, no apparent cultural differences between African and Afro-Caribbean PLWH were found, nor was it obvious that reasons for and against disclosure employed by these PLWH differed substantially from reasons reported in studies conducted elsewhere. It is, however, possible that cultural differences do exist in terms of the relative importance assigned to certain reasons but this remains to be investigated. Taken together, the findings of the qualitative studies with African and Afro-Caribbean diaspora communities suggest that the causes, manifestations, and consequences of HIV-related stigma are relatively ubiquitous, as are the coping mechanisms employed by PLWH to deal with HIV-related stigma and the reasons given for and against disclosure of HIV status.

This claim that HIV-related stigma is relatively universal and that differences across cultures are not major but rather nuanced differences is in line with Ogden and Nyblade (2005) who claim that stigma is "far less varied and context-specific than may have been imagined" (p. 7) and that there are more similarities than differences across contexts in the causes of stigma, the forms stigma takes, and the consequences of stigma. Ogden 
and Nyblade (2005) further support the contention that only nuanced differences emerge between cultures. They too observed that such differences pertain to details such as "the particular form of casual transmission that is most commonly feared, or the particular places where that fear manifests itself" (p. 15). Likewise, van Brakel (2006) claims that the consequences of stigma are remarkably similar across cultures and conditions and that all stigma stems from a similar underlying concept. This is supported by Neuberg, Smith, and Asher (2000) who claim that stigmatization processes are ubiquitous because they serve fundamental biological and group needs that have existed and continue to exist across cultures. More specifically, they claim that because group living is necessary for human survival, people will stigmatize those who threaten the effective functioning of the group. In terms of HIV, PLWH around the world can be construed to threaten their respective communities with their potential to infect others physically (contagion) and morally (norm-violation). Indeed, it seems that, in all cultures, there is a fear dimension and moral dimension to HIV-related stigma.

This contention also has implications for theory development and future research. In particular, it suggests that cross-cultural research efforts should no longer focus on qualitatively exploring potential cultural differences in the broad strokes of HIV-related stigma; rather, future research should focus on specific gaps in the literature, and investigate, both qualitatively and quantitatively, nuanced differences between cultures in, for example, the relative importance assigned to reasons for and against disclosure.

\section{PRACTICAL IMPLICATIONS}

The findings of the studies reported in this dissertation contribute not only to our theoretical understanding of HIV-related stigma but also have implications for the practice of HIVrelated stigma reduction. In general, multi-faceted interventions that are geared to multiple levels are advocated (Bos, Schaalma, \& Pryor, 2008; L. Brown, Macintyre, \& Trujillo, 2003; Heijnders \& Van Der Meij, 2006; Mahajan et al., 2008). The study findings reported in this dissertation support this. In Chapter 2 , the findings point to the need for theory and evidencebased interventions that are geared to changing the beliefs that contribute to HIV-related stigma, and that involve PLWH and their communities throughout intervention development, implementation, and evaluation. In particular, Chapter 2 recommends the implementation of community-based interventions that not only provide information and increase knowledge regarding HIV transmission, prevention, and course, but also bring community members in direct or vicarious contact with HIV-positive individuals so as to create familiarity with, dispel myths about, and generate compassion for PLWH. Providing a safe environment in 
which communities and individuals can reflect on and discuss their stigma-related beliefs, attitudes, and values is also considered imperative, as are interventions that seek to change societal and community structures that promote stigmatization.

The findings of Chapter 2 further suggest that, in order to reduce HIV-related stigma, taboos on talking about HIV and sexuality need to be broken. One strategy that can promote this is public disclosure on the part of PLWH (Paxton, 2002a). This, however, should not be done in the absence of adequate support and training (Paxton, 2002b). In fact, support for PLWH is imperative, especially given the findings of Chapters 3,5 , and 6 . These three chapters have shown that PLWH do endure negative psychological, social, and health consequences as a result of HIV-related stigma. Chapters 3 and 6 have also shown that social support can function as an important buffer against the negative consequences of having experienced stigmatization. Additionally, the findings of Chapter 4 indicate that the need for social support is, for many PLWH, substantial enough to risk stigmatization and disclose their HIV status. They further suggest that PLWH need to be supported in their disclosure decision processes. Lastly, Chapter 6 has shown that particularly PLWH with visible symptoms are in need of support. Evidently, a major practical implication of the study findings in this dissertation is that PLWH must be provided with adequate social and emotional support.

An additional practical implication is that the negative consequences of HIV-related stigma for PLWH can be reduced best by targeting the settings and manifestations in which the impact is greatest. In particular, the findings of Chapter 5 suggest that interventions that target families and health care settings are warranted. The setting-specific manifestations established in this study, namely being advised conceal one's status, avoidance, and exaggerated kindness on the part of PLWH's families and awkward social interaction in the health care sector may, in many cases, not be the result of negative intentions and stigmatizing attitudes but rather a lack of interaction skills and/or familiarity with HIV and PLWH. The development of social interaction skills must therefore be a fundamental component of these HIV-related stigma reduction interventions geared to families and the health care sector.

\section{REFLECTIONS ON METHODOLOGY}

Before providing recommendations for future research, it is important to briefly reflect on the methods employed to investigate HIV-related stigma in the studies described in this dissertation. A primary strength of this dissertation is its use of both qualitative 
and quantitative methods. This mixed methods approach utilized the strengths of both qualitative and quantitative methods and allowed for both inductive exploration and deductive investigation (Creswell, 2009). The qualitative studies have offered a rich and contextualized understanding of what it means to have HIV in African and AfroCaribbean communities while the quantitative studies tested hypotheses concerning the psychological and social impact of setting-specific manifestations and certain forms of disclosure. Together, they have furthered our understanding of HIV-related stigma. Another methodological strength is the prominent, but not exclusive, focus on the perspective of the stigmatized individual. Until recently, the perspective of the stigmatized individual has been neglected in social psychological studies of stigma. This is a serious impediment to our understanding of HIV-related stigma. By emphasizing the perspective of the stigmatized individual, and triangulating that perspective with the perspective of perceivers, this dissertation has contributed to reducing the paucity of literature that takes the perspective of the target without neglecting the important role of the perceiver.

A limitation of the methods employed in this dissertation is the cross-sectional nature of the data. Both the qualitative and quantitative studies acquired data from participants on only one occasion. This makes it difficult to draw conclusions about causality in quantitative studies and determine whether themes are temporal or long-lasting in qualitative studies. Longitudinal data acquisition would have been more advantageous. A second limitation pertaining to the quantitative findings is the relatively low response rate to the survey used in these studies. Despite efforts to increase response rates (e.g. personal contact and follow-up reminders) and the successful inclusion of $6 \%$ of all diagnosed PLWH in the Netherlands, the end response rate for the studies reported in the second part of this dissertation was $29.5 \%$. The potential for non-response bias can, therefore, not be dismissed.

\section{RECOMMENDATIONS FOR FUTURE RESEARCH}

Clearly, this dissertation has contributed to our theoretical understanding of HIV-related stigma and provided guidelines for the practice of stigma reduction but the task is not complete. Gaps in our knowledge and understanding of HIV-related stigma and how it can best be reduced remain. For example, more research on the consequences of HIV-related stigma is necessary. This dissertation has focused on the personal psychological and social consequences of HIV-related stigma for PLWH but not on its impact on the broader community and society as a whole. HIV-related stigma has previously been established as a barrier to testing, prevention, and adherence (Chesney \& Smith, 1999; Meiberg, Bos, 
Onya, \& Schaalma, 2008; UNAIDS, 2009; Vermeer, Bos, Mbwambo, Kaaya, \& Schaalma, 2009), but more research in this regard is necessary. In particular, it would be worthwhile to investigate how HIV-related stigma and the corresponding taboos on talking about HIV contribute to sexual risk-taking.

Additionally, future research needs to explore, in more detail than has been the case in this dissertation, how PLWH cope with HIV-related stigma. Understanding the extent to which a number of coping strategies are employed by PLWH is particularly important because some coping strategies mitigate the negative consequences of stigma better than others do. For example, coping strategies such as support seeking and positive reappraisal have been found to be positively related to psychological well-being while stigma avoidance has been found to yield greater psychological distress (Gonzalez, Solomon, Zvolensky, \& Miller, 2009; Kraaij et al., 2008). It is, therefore, important that we identify the extent to which the various coping strategies are employed and confirm which strategies are most beneficial. With that information, we will be better able to develop interventions that support PLWH and promote the use of the most advantageous coping strategies.

Another area of research that should be expanded pertains to social interactions between perceivers and targets of stigmatization (Hebl \& Dovidio, 2005). We know that direct contact between PLWH and others can function to reduce stigmatization (Herek \& Capitanio, 1997; Pettigrew \& Tropp, 2006), but we know too little about which particular interaction strategies are most beneficial to the reduction of HIV-related stigma and the provision of social support. Both perceivers and targets can influence, through their verbal and non-verbal communication, the likelihood and extent of stigmatizing behavior in social interactions (Bos, 2001; Bos, Dijker, \& Koomen, 2007; Hebl \& Dovidio, 2005). The technology of virtual reality offers a new and innovative environment in which interaction strategies can be tested and compared (Dotsch \& Wigboldus, 2008; Yee, Bailenson, Urbanek, Chang, \& Merget, 2007), and is thus worthy of exploration.

Further, research on the effectiveness of HIV-related stigma reduction interventions is necessary. There is now a large body of literature on the experience of stigma but too few studies have evaluated the process, impact, or effectiveness of stigma reduction interventions (for exceptions, see Markham et al., 2000; Yiu, Mak, Ho, \& Chui, 2010). This is a serious deficit in HIV-related stigma research and practice. Rather than just trying strategies out and hoping for the best, we need to establish what works and what works best on all levels of intervention (intrapersonal, interpersonal, community, and institutional levels). Comprehensive and detailed evaluations of stigma reduction interventions and, in particular, of their respective components are therefore warranted (Abraham \& Michie, 
2008; Bos, Schaalma, \& Pryor, 2008; Schaalma \& Kok, 2009). Such evaluation studies should be initiated not post-hoc but rather from the very inception of an intervention, and in collaboration with targets and relevant stakeholders (Bartholomew, Parcel, Kok, \& Gottlieb, 2006; Bos, Schaalma, \& Pryor, 2008).

Also, given the dynamic nature of the HIV epidemic, changes in HIV-related stigma over time need to be documented (Van Brakel, 2006). Too few studies have investigated HIVrelated stigma longitudinally (for exceptions, see Herek, Capitanio, \& Widaman, 2002; Maughan-Brown, 2009). Longitudinal investigations are now particularly important as the epidemic undergoes fundamental changes. In developing countries, HIV is changing from a terminal to a chronic condition due to recent mass roll-outs of antiretroviral therapy and, in developed countries, where therapy has been available for some time, many PLWH are entering old age with all its accompanying complications. Obviously, changes in the epidemic can yield changes in the experience and consequences of HIV-related stigma. These changes need to be documented.

Further, in the shadow of the vast amount of research on public and self stigma, there are two other forms of stigma that need to be studied more extensively. These are stigma-byassociation and institutional stigma (Pryor \& Reeder, in press). With respect to stigma-byassociation, future research can be informed by our current knowledge regarding public and self stigma. As such, it would be wise to study which manifestations in particular settings are experienced by people subjected to stigma-by-association, the impact of those manifestations on the psychological and social well-being of people subjected to stigma-byassociation, how people cope with stigma-by-association, how people decide whether or not to disclose their association with a stigmatized individual, and also the extent to which their association can be hidden and the impact of this. Additionally, the dynamics underlying stigma-by-association and the targets most affected by stigma-by-association should be established. With respect to institutional HIV-related stigma, it would likely be beneficial to incorporate in our social psychological and sociological body of literature research from other domains (law, economics, policy research) where expertise in ascertaining how legal, economic, and structural factors contribute to the promotion and perpetuation of institutional stigma is present. A multi-disciplinary approach has much to offer.

A final recommendation for future research pertains to the very concept of stigma. Conceptually, stigma remains, at least to some degree, vague. There are number of related concepts and it is often unclear whether stigma is fundamentally different than, for example, deviance or labeling (Dijker \& Koomen, 2007). Future research should therefore endeavor to create more conceptual clarity on the differences and similarities between 
stigma or components of stigma and deviance, labeling, marginality, stereotyping, prejudice, and discrimination. To some extent, this has already been done. Traditionally, the social psychological literature has claimed that stereotyping represents cognitions, prejudice emotions, and discrimination behaviors, and that stigma comprises all three components (Dijker \& Koomen, 2003; Earnshaw \& Chaudoir, 2009) but one can ask whether it is functional to employ a number of possibly interchangeable terms to describe what may be the same phenomenon. It would be both interesting and beneficial if experts in the fields of stigma, deviance, labeling, and prejudice were to engage in dialogue and debate on the similarities and differences between these concepts.

\section{CONCLUDING REMARKS}

This dissertation has investigated the social and psychological processes underlying, and resulting from, HIV-related stigma. It has explored, using both qualitative and quantitative methods, aspects of public and self stigma and has taken predominantly, but not exclusively, the perspective of the stigmatized individual. It has further focused on populations disproportionately affected by HIV and HIV-related stigma, namely African and Afro-Caribbean communities in the Netherlands, and explored in detail more specific aspects of HIV-related stigma with a broader study population. It has delineated the beliefs contributing to, manifestations and consequences of, and coping mechanisms for HIVrelated stigma and discussed issues of disclosure, and in doing so, has contributed to a better understanding of what it means to live with HIV. 
GENERAL DISCUSSION

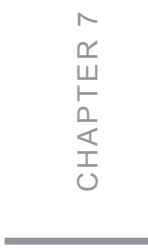


REFERENCES 
Abraham, C., \& Michie, S. (2008). A taxonomy of behavior change techniques used in interventions. Health Psychology, 27(3), 379-387.

Alonzo, A. A., \& Reynolds, N. R. (1995). Stigma, HIV and AIDS: An exploration and elaboration of a stigma trajectory. Social Science and Medicine, 41(3), 303-315.

Anderson, J., \& Doyal, L. (2004). Women from Africa living with HIV in London: A descriptive study. AIDS Care, 16(1), 95-105.

Anderson, M., Elam, G., Gerver, S., Solarin, I., Fenton, K., \& Easterbrook, P. (2008). HIVI AIDS-related stigma and discrimination: Accounts of HIV-positive Caribbean people in the United Kingdom. Social Science and Medicine, 67(5), 790-798.

Anderson, M., Elam, G., Solarin, I., Gerver, S., Fenton, K., \& Easterbrook, P. (2009). Coping with HIV: Caribbean people in the United Kingdom. Qualitative Health Research, 19(8), 1060-1075.

Andrewin, A., \& Chien, L. Y. (2008). Stigmatization of patients with HIV/AIDS among doctors and nurses in Belize. AIDS Patient Care and STDs, 22(11), 897-906.

Baron, R. M., \& Kenny, D. A. (1986). The moderator-mediator variable distinction in social psychological research: Conceptual, strategic, and statistical considerations. Journal of Personality and Social Psychology, 51(6), 1173-1182.

Bartholomew, L. K., Parcel, G. S., Kok, G., \& Gottlieb, N. H. (2006). Planning health promotion programs: An intervention mapping approach (2nd ed.). San Francisco, CA: Jossey-Bass.

Baumeister, R. F., Leary, M. R., Higgins, E. T., \& Kruglanski, A. W. (2000). The need to belong: Desire for interpersonal attachments as a fundamental human motivation. In E. T. Higgins \& A. W. Kruglanski (Eds.), Motivational science: Social and personality perspectives. (pp. 24-49). New York, NY: Psychology Press.

Berger, B. E., Ferrans, C. E., \& Lashley, F. R. (2001). Measuring stigma in people with HIV: Psychometric assessment of the HIV stigma scale. Research in Nursing \& Health, 24(6), 518-529.

Bermingham, S., \& Kippax, S. (1998). HIV-related discrimination: A survey of New South Wales general practitioners. Australian and New Zealand Journal of Public Health, 22(1), 92-97.

Bing, E. G., Burnam, M. A., Longshore, D., Fleishman, J. A., Sherbourne, C. D., London, A. S., et al. (2001). Psychiatric disorders and drug use among human immunodeficiency virus-infected adults in the United States. Archives of General Psychiatry, 58(8), 721-728.

Black, B. P., \& Miles, M. S. (2002). Calculating the risks and benefits of disclosure in African American women who have HIV. Journal of Obstetric, Gynecologic \& Neonatal Nursing, 31(6), 688-697. 
Bond, V., Chase, E., \& Aggleton, P. (2002). Stigma, HIVIAIDS and prevention of mother-tochild transmission in Zambia. Evaluation and Program Planning, 25(4), 347-356.

Bos, A. E. R. (2001). HIV stigma and social interaction: Examining strategies to influence perceivers' emotional and behavioral reactions to initial encounters. Maastricht: Maastricht University.

Bos, A. E. R., Dijker, A. J. M., \& Koomen, W. (2007). Sex differences in emotional and behavioral responses to HIV+ individuals' expression of distress. Psychology \& Health, 22(4), 493-511.

Bos, A. E. R., Kanner, D., Muris, P., Janssen, B., \& Mayer, B. (2009). Mental illness stigma and disclosure: Consequences of coming out of the closet. Issues in Mental Health Nursing, 30(8), 509-513.

Bos, A. E. R., Kok, G., \& Dijker, A. J. (2001). Public reactions to people with HIVIAIDS in the Netherlands. AIDS Education and Prevention, 13(3), 219-228.

Bos, A. E. R., Schaalma, H. P., \& Pryor, J. B. (2008). Reducing AIDS-related stigma in developing countries: The importance of theory- and evidence-based interventions. Psychology, Health \& Medicine, 13(4), 450-460.

Brooks, R. A., Etzel, M. A., Hinojos, E., Henry, C. L., \& Perez, M. (2005). Preventing HIV among Latino and African American gay and bisexual men in a context of HIVrelated stigma, discrimination, and homophobia: perspectives of providers. AIDS Patient Care and STDs, 19(11), 737-744.

Brown, L., Macintyre, K., \& Trujillo, L. (2003). Interventions to reduce HIVIAIDS stigma: What have we learned? AIDS Education and Prevention, 15(1), 49-69.

Brown, R., \& Hewstone, M. (2005). An integrative theory of intergroup contact. In M. P. Zanna (Ed.), Advances in Experimental Social Psychology (Vol. 37, pp. 255-343). San Diego, CA: Elsevier Academic Press.

Burns, F. M., Imrie, J. Y., Nazroo, J., Johnson, A. M., \& Fenton, K. A. (2007). Why the(y) wait? Key informant understandings of factors contributing to late presentation and poor utilization of HIV health and social care services by African migrants in Britain. AIDS Care, 19(1), 102-108.

Buzzella, B. A., Beals, K., \& Peplau, L. A. (2003). The impact of voluntary disclosure on the psychological well-being of homosexual individuals. UCLA Undergraduate Psychology Journal, 2(1).

Calin, T., Green, J., Hetherton, J., \& Brook, G. (2007). Disclosure of HIV among black African men and women attending a London HIV clinic. AIDS Care, 19(3), 385-391.

Campbell, C., \& Deacon, H. (2006). Unravelling the contexts of stigma: From internalisation to resistance to change. Journal of Community \& Applied Social Psychology, 16(6), 411-417. 
Campbell, C., Foulis, C. A., Maimane, S., \& Sibiya, Z. (2005). "I have an evil child at my house": Stigma and HIVIAIDS management in a South African community. American Journal of Public Health, 95(5), 808-815.

Carr, R. L. (2004). Stigmas, coping and the impact of gender on patterns of social ostracism: A qualitative study with HIV+ Jamaicans. Kingston, Jamaica: University of the West Indies.

Carr, R. L., \& Gramling, L. F. (2004). Stigma: A health barrier for women with HIVIAIDS. Journal of the Association of Nurses in AIDS Care, 15(5), 30-39.

Chandra, P. S., Deepthivarma, S., \& Manjula, V. (2003). Disclosure of HIV infection in south India: Patterns, reasons and reactions. AIDS Care, 15(2), 207-215.

Chesney, M. A., \& Smith, A. W. (1999). Critical delays in HIV testing and care: The potential role of stigma. American Behavioral Scientist, 42(7), 1162-1174.

Clark, H. J., Lindner, G., Armistead, L., \& Austin, B. J. (2003). Stigma, disclosure, and psychological functioning among HIV-infected and non-infected African-American women. Women \& Health, 38(4), 57-71.

Corrigan, P. W., \& Matthews, A. K. (2003). Stigma and disclosure: Implications for coming out of the closet. Journal of Mental Health, 12(3), 235-248.

Crandall, C. S., \& Coleman, R. (1992). AIDS-related stigmatization and the disruption of social relationships. Journal of Social and Personal Relationships, 9(2), 163-177.

Creswell, J. W. (2009). Research design: Qualitative, quantitative, and mixed method approaches (3rd ed.). Thousand Oaks, CA: Sage.

Crocker, J., Major, B., \& Steele, C. (1998). Social stigma. In D. T. Gilbert, S. T. Fiske \& G. Lindzey (Eds.), The handbook of social psychology (4th ed., Vol. 1 \& 2, pp. 504553). New York, NY: McGraw-Hill.

de-Graft Aikins, A. (2006). Reframing applied disease stigma research: A multilevel analysis of diabetes stigma in Ghana. Journal of Community \& Applied Social Psychology, 16(6), 426-441.

Deacon, H. (2006). Towards a sustainable theory of health-related stigma: Lessons from the HIVIAIDS literature. Journal of Community \& Applied Social Psychology, 16(6), 418-425.

Deacon, H., Stephney, I., \& Prosalendis, S. (2005). Understanding HIV/AIDS stigma: A theoretical and methodological analysis. Capetown, South Africa: Human Sciences Research.

Derlega, V. J., Winstead, B. A., Greene, K., Serovich, J., \& Elwood, W. N. (2004). Reasons for HIV disclosure/nondisclosure in close relationships: Testing a model of HIVdisclosure decision making. Journal of Social and Clinical Psychology, 23(6), 747-767. 
Derlega, V. J., Winstead, B. A., Oldfield, I. E., \& Barbee, A. P. (2003). Close relationships and social support in coping with HIV: A test of sensitive interaction systems theory. AIDS and Behavior, 7(2), 119-129.

Dijker, A. J., \& Koomen, W. (2003). Extending Weiner's attribution-emotion model of stigmatization of ill persons. Basic and Applied Social Psychology, 25(1), 51-68.

Dijker, A. J., \& Koomen, W. (2007). Stigmatization, tolerance, and repair: An integrative psychological analysis of responses to deviance. Cambridge, UK: Cambridge University Press.

Dodds, C. (2006). HIV-related stigma in England: Experiences of gay men and heterosexual African migrants living with HIV. Journal of Community \& Applied Social Psychology, 16(6), 472-480.

Dodds, C., Keogh, P., Chime, O., Haruperi, T., Nabulya, B., SSanya SSeruma, W., et al. (2004). Outsider status: Stigma and discrimination experienced by gay men and African people with HIV. London: Sigma Research.

Dotsch, R., \& Wigboldus, D. H. J. (2008). Virtual prejudice. Journal of Experimental Social Psychology, 44(4), 1194-1198.

Dovidio, J. F., Major, B., \& Crocker, J. (2000). Stigma: Introduction and overview. In T. F. Heatherton, R. E. Kleck, M. R. Hebl \& J. G. Hull (Eds.), The social psychology of stigma (pp. 1-28). New York, NY: Guilford Press.

Dray-Spira, R., Lert, F., Marimoutou, C., Bouhnik, A. D., \& Obadia, Y. (2003). Socioeconomic conditions, health status and employment among persons living with HIVIAIDS in France in 2001. AIDS Care, 15(6), 739-748.

Duffy, L. (2005). Suffering, shame, and silence: The stigma of HIVIAIDS. Journal of the Association of Nurses in AIDS Care, 16(1), 13-20.

Earnshaw, V. A., \& Chaudoir, S. R. (2009). From conceptualizing to measuring HIV stigma: A review of HIV stigma mechanism measures. AIDS and Behavior, 13(6), 1160-1177.

Erwin, J., Morgan, M., Britten, N., Gray, K., \& Peters, B. (2002). Pathways to HIV testing and care by black African and white patients in London. Sexually Transmitted Infections, 78(1), 37-39.

Frable, D. E. S., Platt, L., \& Hoey, S. (1998). Concealable stigmas and positive selfperceptions: Feeling better around similar others. Journal of Personality and Social Psychology, 74(4), 909-922.

Galvan, F. H., Davis, E. M., Banks, D., \& Bing, E. G. (2008). HIV stigma and social support among African Americans. AIDS Patient Care and STDs, 22(5), 423-436.

Gardezi, F., Calzavara, L., Husbands, W., Tharao, W., Lawson, E., Myers, T., et al. (2008). Experiences of and responses to HIV among African and Caribbean communities in Toronto, Canada. AIDS Care, 20(6), 718-725. 
Genberg, B. L., Hlavka, Z., Konda, K. A., Maman, S., Chariyalertsak, S., Chingono, A., et al. (2009). A comparison of HIVIAIDS-related stigma in four countries: Negative attitudes and perceived acts of discrimination towards people living with HIV/ AIDS. Social Science and Medicine, 68(12), 2279-2287.

Gielen, A. C., Fogarty, L., O’Campo, P., Anderson, J., Keller, J., \& Faden, R. (2000). Women living with HIV: Disclosure, violence, and social support. Journal of Urban Health, 77(3), 480-491.

Gielen, A. C., McDonnell, K. A., Burke, J. G., \& O'Campo, P. (2000). Women's lives after an HIV-positive diagnosis: Disclosure and violence. Maternal and Child Health Journal, 4(2), 111-120.

Goffman, I. (1963). Stigma: Notes on the Management of Spoiled Identity. Eaglewood Cliffs, NJ: Prentice-Hall.

Gonzalez, A., Solomon, S. E., Zvolensky, M. J., \& Miller, C. T. (2009). The interaction of mindful-based attention and awareness and disengagement coping with HIVI AIDS-related stigma in regard to concurrent anxiety and depressive symptoms among adults with HIVIAIDS. Journal of Health Psychology, 14(3), 403-413.

Greeff, M., Phetlhu, R., Makoae, L. N., Dlamini, P. S., Holzemer, W. L., Naidoo, J. R., et al. (2008). Disclosure of HIV status: Experiences and perceptions of persons living with HIVIAIDS and nurses involved in their care in Africa. Qualitative Health Research, 18(3), 311-324.

Green, G., \& Platt, S. (1997). Fear and loathing in health care settings reported by people with HIV. Sociology of Health \& IIIness, 19(1), 70-92.

Greenberg, M. A., \& Stone, A. A. (1992). Emotional disclosure about traumas and its relation to health: Effects of previous disclosure and trauma severity. Journal of Personality and Social Psychology, 63(1), 75-84.

Hancock, B. (1998). Trent Focus for research and development in primary health care: An introduction to qualitative research. Nottingham: Trent Focus Group.

Hartwig, K. A., Kissioki, S., \& Hartwig, C. D. (2006). Church leaders confront HIVIAIDS and stigma: A case study from Tanzania. Journal of Community \& Applied Social Psychology, 16(6), 492-497.

Hebl, M. R., \& Dovidio, J. F. (2005). Promoting the 'social' in the examination of social stigmas. Personality and Social Psychology Review, 9(2), 156-182.

Heckman, T. G., Anderson, E. S., Sikkema, K. J., Kochman, A., Kalichman, S. C., \& Anderson, T. (2004). Emotional distress in nonmetropolitan persons living with HIV disease enrolled in a telephone-delivered, coping improvement group intervention. Health Psychology, 23(1), 94-100. 
Heijnders, M., \& Van Der Meij, S. (2006). The fight against stigma: An overview of stigmareduction strategies and interventions. Psychology, Health \& Medicine, 11(3), 353-363.

Herek, G. M. (1999). AIDS and stigma. American Behavioral Scientist, 42(7), 1106-1116.

Herek, G. M., \& Capitanio, J. P. (1996). 'Some of my best friends': Intergroup contact, concealable stigma, and heterosexuals' attitudes toward gay men and lesbians. Personality and Social Psychology Bulletin, 22(4), 412-424.

Herek, G. M., \& Capitanio, J. P. (1997). AIDS stigma and contact with persons with AIDS: Effects of direct and vicarious contact. Journal of Applied Social Psychology, 27(1), 1-36.

Herek, G. M., Capitanio, J. P., \& Widaman, K. F. (2002). HIV-related stigma and knowledge in the United States: Prevalence and trends, 1991-1999. American Journal of Public Health, 92(3), 371-377.

HIV Monitoring Foundation. (2008). Monitoring of Human Immunodeficiency Virus (HIV) infection in the Netherlands. Amsterdam, the Netherlands: HIV Monitoring Foundation.

Joachim, G., \& Acorn, S. (2000). Living with chronic illness: The interface of stigma and normalization. CJNR: Canadian Journal of Nursing Research, 32(3), 37-48.

Jones, E., Farina, A., Hastorf, A., Markus, H., Miller, D., \& Scott, R. (1984). Social stigma: The psychology of marked relationships. New York, NY: W.H. Freeman.

Kalichman, S. C., DiMarco, M., Austin, J., Luke, W., \& DiFonzo, K. (2003). Stress, social support, and HIV-status disclosure to family and friends among HIV-positive men and women. Journal of Behavioral Medicine, 26(4), 315-332.

Kalichman, S. C., \& Simbayi, L. (2004). Traditional beliefs about the cause of AIDS and AIDS-related stigma in South Africa. AIDS Care, 16(5), 572-580.

Kalichman, S. C., \& Simbayi, L. C. (2003). HIV testing attitudes, AIDS stigma, and voluntary HIV counselling and testing in a black township in Cape Town, South Africa. Sexually Transmitted Infections, 79(6), 442-447.

Kalichman, S. C., Simbayi, L. C., Jooste, S., Toefy, Y., Cain, D., Cherry, C., et al. (2005). Development of a brief scale to measure AIDS-related stigma in South Africa. AIDS and Behavior, 9(2), 135-143.

Kang, E., Rapkin, B. D., Remien, R. H., Mellins, C. A., \& Oh, A. (2005). Multiple dimensions of HIV stigma and psychological distress among Asians and Pacific Islanders living with HIV illness. AIDS and Behavior, 9(2), 145-154.

Kempen, G. I. J. M., \& Van Eijk, L. M. (1995). The psychometric properties of he SSL12I, a short scale for measuring social support in the elderly. Social Indicators Research, 35(3), 303-312. 
Kinniburgh, J., Scott, P., Gottlieb, M., \& Power, L. (2001). Prejudice, Discrimination and HIV. London, UK: Terrence Higgins Trust.

Kraaij, V., van der Veek, S. M. C., Garnefski, N., Witlox, R., Maes, S., \& Schroevers, M. (2008). Coping, goal adjustment, and psychological well-being in HIV-infected men who have sex with men. AIDS Patient Care and STDs, 22(5), 395-402.

Kumar, A., Waterman, I., Kumari, G., \& Carter, A. O. (2006). Prevalence and correlates of HIV serostatus disclosure: A prospective study among HIV-infected postparturient women in Barbados. AIDS Patient Care and STDs, 20(10), 724-730.

Lam, P. K., Naar-King, S., \& Wright, K. (2007). Social support and disclosure as predictors of mental health in HIV-positive youth. AIDS Patient Care and STDs, 21(1), 20-29.

Landau, G., \& York, A. S. (2004). Keeping and disclosing a secret among people with HIV in Israel. Health and Social Work, 29(2), 116-126.

Lee, J. D., \& Craft, E. A. (2002). Protecting one's self from a stigmatized disease...once one has it. Deviant Behavior, 23(3), 267-299.

Lee, R. S., Kochman, A., \& Sikkema, K. J. (2002). Internalized stigma among people living with HIV-AIDS. AIDS and Behavior, 6(4), 309-319.

Li, L., Lee, S. J., Thammawijaya, P., Jiraphongsa, C., \& Rotheram-Borus, M. J. (2009). Stigma, social support, and depression among people living with HIV in Thailand. AIDS Care, 21(8), 1007-1013.

Lichtenstein, B., Laska, M. K., \& Clair, J. M. (2002). Chronic sorrow in the HIV-positive patient: Issues of race, gender, and social support. AIDS Patient Care and STDs, 16(1), 27-38.

Link, B. G., \& Phelan, J. C. (2001). Conceptualizing stigma. Annual Review of Sociology, 27, 363-385.

Mahajan, A. P., Sayles, J. N., Patel, V. A., Remien, R. H., Sawires, S. R., Ortiz, D. J., et al. (2008). Stigma in the HIVIAIDS epidemic: A review of the literature and recommendations for the way forward. AIDS, 22 Supplement 2, S67-79.

Major, B., \& O'Brien, L. T. (2005). The social psychology of stigma. Annual Review of Psychology, 56, 393-421.

Mak, W. W., Cheung, R. Y., Law, R. W., Woo, J., Li, P. C., \& Chung, R. W. (2007). Examining attribution model of self-stigma on social support and psychological well-being among people with HIV+/AIDS. Social Science and Medicine, 64(8), 1549-1559.

Makoae, L. N., Greeff, M., Phetlhu, R. D., Uys, L. R., Naidoo, J. R., Kohi, T. W., et al. (2008). Coping with HIV-related stigma in five African countries. Journal of the Association of Nurses in AIDS Care, 19(2), 137-146.

Malcolm, A., Aggleton, P., Bronfman, M., Galvão, J., Mane, P., \& Verral, J. (1998). HIVrelated stigmatization and discrimination: Its forms and contexts. Critical Public Health, 8(4), 347-370. 
Maman, S., Abler, L., Parker, L., Lane, T., Chirowodza, A., Ntogwisangu, J., et al. (2009). A comparison of HIV stigma and discrimination in five international sites: The influence of care and treatment resources in high prevalence settings. Social Science and Medicine, 68(12), 2271-2278.

Markham, C., Baumler, E., Richesson, R., Parcel, G., Basen-Engquist, K., Kok, G., et al. (2000). Impact of HIV-positive speakers in a multicomponent, school-based HIVISTD prevention program for inner-city adolescents. AIDS Education and Prevention, 12(5), 442-454.

Maughan-Brown, B. (2009). Stigma rises despite antiretroviral roll-out: A longitudinal analysis in South Africa. Social Science and Medicine, 70(3), 368-374.

Medley, A. M., Kennedy, C. E., Lunyolo, S., \& Sweat, M. D. (2009). Disclosure outcomes, coping strategies, and life changes among women living with HIV in Uganda. Qualitative Health Research, 19(12), 1744-1754.

Meiberg, A. E., Bos, A. E., Onya, H. E., \& Schaalma, H. P. (2008). Fear of stigmatization as barrier to voluntary HIV counselling and testing in South Africa. East African Journal of Public Health, 5(2), 49-54.

Melchert, T. P., \& Patterson, M. M. (1999). Duty to warn and intervention with HIV-positive clients. Professional Psychology: Research and Practice, 30(2), 180-186.

Miller, C. T., \& Kaiser, C. R. (2001). A theoretical perspective on coping with stigma. Journal of Social Issues, 57(1), 73-92.

Miller, C. T., \& Major, B. (2000). Coping with stigma and prejudice. In T. F. Heatherton, R. E. Kleck, M. R. Hebl \& J. G. Hull (Eds.), The social psychology of stigma. (pp. 243-272). New York, NY US: Guilford Press.

Mills, E. A. (2006). From the physical self to the social body: Expressions and effects of HIV-Related stigma in South Africa. Journal of Community \& Applied Social Psychology, 16(6), 498-503.

Monico, A. M., Tanga, E. O., \& Nuwagaba, A. (2001). Uganda: HIV and AIDS-related discrimination, stigmatization and denial (No. UNAIDS/01.43E). Geneva, Switzerland: UNAIDS.

Neuberg, S. L., Smith, D. M., \& Asher, T. (2000). Why people stigmatize: Toward a biocultural framework. In T. F. Heatherton, R. E. Kleck, M. R. Hebl \& J. G. Hull (Eds.), The social psychology of stigma (pp. 31-61). New York, NY: Guilford Press.

Neuberg, S. L., Smith, D. M., Hoffman, J. C., \& Russell, F. J. (1994). When we observe stigmatized and 'normal' individuals interacting: Stigma by association. Personality and Social Psychology Bulletin, 20(2), 196-209.

Norman, L. R., Abreu, S., Candelaria, E., \& Sala, A. (2009). The effect of sympathy on discriminatory attitudes toward persons living with HIVIAIDS in Puerto Rico: A hierarchical analysis of women living in public housing. AIDS Care, 21(2), 140-149. 
Nyblade, L., Pande, R., Mathur, S., MacQuarrie, K., \& Kidd, R. (2003). Disentangling HIV and AIDS stigma in Ethiopia, Tanzania and Zambia. Washington, DC: International Center for Research on Women.

Ogden, J., \& Nyblade, L. (2005). Common at its core: HIV-related stigma across contexts. Washington, DC: International Center for Research on Women (ICRW).

Okoror, T. A., Airhihenbuwa, C. O., Zungu, M., Makofani, D., Brown, D. C., \& Iwelunmor, J. (2007). "My mother told me I must not cook anymore": Food, culture, and the context of HIV- and AIDS-related stigma in three communities in South Africa. International Quarterly of Community Health Education, 28(3), 201-213.

Oyserman, D., \& Swim, J. K. (2002). Stigma: An Insider's View. Journal of Social Issues, $57(1), 1-14$

Pachankis, J. E. (2007). The psychological implications of concealing a stigma: A cognitiveaffective-behavioral model. Psychological Bulletin, 133(2), 328-345.

Parker, R., \& Aggleton, P. (2003). HIV and AIDS-related stigma and discrimination: A conceptual framework and implications for action. Social Science and Medicine, 57(1), 13-24.

Parsons, J. T., VanOra, J., Missildine, W., Purcell, D., \& Gomez, C. (2004). Positive and negative consequences of HIV disclosure among seropositive injection drug users. AIDS Education and Prevention, 16(5), 459-475.

Paxton, S. (2002a). The impact of utilizing HIV-positive speakers in AIDS education. AIDS Education and Prevention, 14(4), 282-294.

Paxton, S. (2002b). The paradox of public HIV disclosure. AIDS Care, 14(4), 559-567.

Pence, B. W., Miller, W. C., Whetten, K., Eron, J. J., \& Gaynes, B. N. (2006). Prevalence of DSM-IV-defined mood, anxiety, and substance use disorders in an HIV clinic in the Southeastern United States. JAIDS Journal of Acquired Immune Deficiency Syndromes, 42(3), 298-306.

Pennebaker, J. W., Colder, M., \& Sharp, L. K. (1990). Accelerating the coping process. Journal of Personality and Social Psychology, 58(3), 528-537.

Pettigrew, T. F., \& Tropp, L. R. (2006). A meta-analytic test of intergroup contact theory. Journal of Personality and Social Psychology, 90(5), 751-783.

Polit, D. F., \& Beck, C. T. (2010). Essentials of nursing research. Philidelphia, PA: Wolters Kluwer Lippincott Williams \& Wilkins.

Pryor, J. B., \& Reeder, G. D. (in press). HIV-related stigma. In B. Hall, J. Hall \& C. Cockerell (Eds.), AIDS: The first 30 years. Shelton, CT: People's Medical.

Pryor, J. B., Reeder, G. D., \& Landau, S. (1999). A social-psychological analysis of HIV-related stigma: A two-factor theory. American Behavioral Scientist, 42(7), 1193-1211. 
Pryor, J. B., Reeder, G. D., Yeadon, C., \& Hesson-McLnnis, M. (2004). A dual-process model of reactions to perceived stigma. Journal of Personality and Social Psychology, 87(4), 436-452.

Quinn, D. M. (2006). Concealable versus conspicuous stigmatized identities. In S. Levin \& C. van Laar (Eds.), Stigma and group inequality: Social psychological approaches (pp. 83-103). Mahwah, NJ: Lawrence Erlbaum.

Rankin, W. W., Brennan, S., Schell, E., Laviwa, J., \& Rankin, S. H. (2005). The stigma of being HIV-positive in Africa. PLoS Med, 2(8), e247.

Reidpath, D. D., \& Chan, K. Y. (2005). A method for the quantitative analysis of the layering of HIV-related stigma. AIDS Care, 17(4), 425-432.

Remennick, L. (2000). Childless in the land of imperative motherhood: Stigma and coping among infertile Israeli women. Sex Roles, 43(11), 821-841.

Reynolds, N. R., Neidig, J. L., Wu, A. W., Gifford, A. L., \& Holmes, W. C. (2006). Balancing disfigurement and fear of disease progression: Patient perceptions of HIV body fat redistribution. AIDS Care, 18(7), 663-673.

Riggs, S. A., Vosvick, M., \& Stallings, S. (2007). Attachment style, stigma and psychological distress among HIV+ adults. Journal of Health Psychology, 12(6), 922-936.

Rintamaki, L. S., Davis, T. C., Skripkauskas, S., Bennett, C. L., \& Wolf, M. S. (2006). Social stigma concerns and HIV medication adherence. AIDS Patient Care and STDs, 20(5), 359-368.

Rintamaki, L. S., Scott, A. M., Kosenko, K. A., \& Jensen, R. E. (2007). Male patient perceptions of HIV stigma in health care contexts. AIDS Patient Care and STDs, 21(12), 956-969.

Rosenberg, M. (1965). Society and the adolescent self-image. Princeton, NJ: University Press.

Rosenthal, T. L., Downs, J. M., Arheart, K. L., \& Deal, N. (1991). Similarities and differences on five inventories among mood and anxiety disorder patients. Behaviour Research and Therapy, 29(3), 239-247.

Roura, M., Urassa, M., Busza, J., Mbata, D., Wringe, A., \& Zaba, B. (2008). Scaling up stigma? The effects of antiretroviral roll-out on stigma and HIV testing. Early evidence from rural Tanzania. Sexually Transmitted Infections, 85(4), 308-312.

Rozin, P., Markwith, M., \& Nemeroff, C. (1992). Magical contagion beliefs and fear of AIDS. Journal of Applied Social Psychology, 22(14), 1081-1092.

Sanches, R. S., Mill, J., Machado, A. A., Donadi, E. A., \& Morais Fernandes, A. P. (2009). Facial lipoatrophy: Appearances are not deceiving. Journal of the Association of Nurses in AIDS Care, 20(3), 169-175. 
Sandelowski, M., Lambe, C., \& Barroso, J. (2004). Stigma in HIV-positive women. Journal of Nursing Scholarship, 36(2), 122-128.

Sayles, J. N., Ryan, G. W., Silver, J. S., Sarkisian, C. A., \& Cunningham, W. E. (2007). Experiences of social stigma and implications for healthcare among a diverse population of HIV positive adults. Journal of Urban Health, 84(6), 814-828.

Scambler, G., \& Hopkins, A. (1986). Being epileptic: Coming to terms with stigma. Sociology of Health \& IIIness, 8(1), 26-43.

Scambler, G., \& Paoli, F. (2008). Health work, female sex workers and HIVIAIDS: Global and local dimensions of stigma and deviance as barriers to effective interventions. Social Science and Medicine, 66(8), 1848-1862.

Schaalma, H., \& Kok, G. (2009). Decoding health education interventions: The times are a-changin.' Psychology \& Health, 24(1), 5-9.

Schmitt, D. P., \& Allik, J. (2005). Simultaneous administration of the Rosenberg Self-Esteem Scale in 53 nations: Exploring the universal and culture-specific features of global self-esteem. Journal of Personality and Social Psychology, 89(4), 623-642.

Serovich, J. M. (2001). A test of two HIV disclosure theories. AIDS Education and Prevention, 13(4), 355-364.

Serovich, J. M., \& Mosack, K. E. (2003). Reasons for HIV disclosure or nondisclosure to casual sexual partners. AIDS Education and Prevention, 15(1), 70-80.

Shamos, S., Hartwig, K. A., \& Zindela, N. (2009). Men's and women's experiences with HIV and stigma in Swaziland. Qualitative Health Research, 19(12), 1678-1689.

Shiripinda, I., \& van Eerdewijk, A. (2008). Facing HIV in the Netherlands: Lived experiences of migrants living with HIV. Utrecht, the Netherlands: Pharos/SOA AIDS.

Simbayi, L. C., Kalichman, S., Strebel, A., Cloete, A., Henda, N., \& Mqeketo, A. (2007). Internalized stigma, discrimination, and depression among men and women living with HIVIAIDS in Cape Town, South Africa. Social Science and Medicine, 64(9), 1823-1831.

Simoni, J. M., Mason, H. R., \& Marks, G. (1997). Disclosing HIV status and sexual orientation to employers. AIDS Care, 9(5), 589-599.

Simoni, J. M., Mason, H. R. C., Marks, G., Ruiz, M. S., Reed, D., \& Richardson, J. L. (1995). Women's self-disclosure of HIV infection: Rates, reasons, and reactions. Journal of Consulting and Clinical Psychology, 63(3), 474-478.

Smart, L., \& Wegner, D. M. (1999). Covering up what can't be seen: Concealable stigma and mental control. Journal of Personality and Social Psychology, 77(3), 474-486.

Smart Richman, L., \& Leary, M. R. (2009). Reactions to discrimination, stigmatization, ostracism, and other forms of interpersonal rejection: A multimotive model. Psychological Review, 116(2), 365-383. 
Smith, R., Rossetto, K., \& Peterson, B. L. (2008). A meta-analysis of disclosure of one's HIV-positive status, stigma and social support. AIDS Care, 20(10), 1266-1275.

Stangor, C., \& Crandall, C. S. (2000). Threat and the social construction of stigma. In T. F. Heatherton, R. E. Kleck, M. R. Hebl \& J. G. Hull (Eds.), The social psychology of stigma. New York, NY: Guilford Press.

Stempel, R. R., Moulton, J. M., \& Moss, A. R. (1995). Self-disclosure of HIV-1 antibody test results: The San Francisco General Hospital cohort. AIDS Education and Prevention, 7(2), 116-123.

Steward, W. T., Herek, G. M., Ramakrishna, J., Bharat, S., Chandy, S., Wrubel, J., et al. (2008). HIV-related stigma: Adapting a theoretical framework for use in India. Social Science and Medicine, 67(8), 1225-1235.

Stutterheim, S. E., Bos, A. E. R., \& Schaalma, H. P. (2008). HIV-related stigma in the Netherlands. Maastricht, the Netherlands: AIDS Fonds \& Maastricht University.

Stutterheim, S. E., Pryor, J. B., Bos, A. E., Hoogendijk, R., Muris, P., \& Schaalma, H. P. (2009). HIV-related stigma and psychological distress: The harmful effects of specific stigma manifestations in various social settings. AIDS, 23(17), 2353-2357.

Swendeman, D., Rotheram-Borus, M. J., Comulada, S., Weiss, R., \& Ramos, M. E. (2006). Predictors of HIV-related stigma among young people living with HIV. Health Psychology, 25(4), 501-509.

Tate, D. C., Van Den Berg, J. J., Hansen, N. B., Kochman, A., \& Sikkema, K. J. (2006). Race, social support, and coping strategies among HIV-positive gay and bisexual men. Culture, Health and Sexuality, 8(3), 235-249.

Trickett, E. J. (2009). Community psychology: Individuals and interventions in community context. Annual Review of Psychology, 60, 395-419.

Ullrich, P. M., Lutgendorf, S. K., \& Stapleton, J. T. (2003). Concealment of homosexual identity, social support and CD4 cell count among HIV-seropositive gay men. Journal of Psychosomatic Research, 54(3), 205-212.

UNAIDS. (2008). 2008 Report on the global AIDS epidemic (No. 08.25E/JC1510E). Geneva, Switzerland: UNAIDS.

UNAIDS. (2009). AIDS epidemic update 2009 (No. UNAIDS/09.36E/JC1700E). Geneva, Switzerland: UNAIDS.

Valle, M., \& Levy, J. (2009). Weighing the consequences: Self-disclosure of HIV-positive status among African American injection drug users. Health Education \& Behavior, 36(1), 155-166.

Van Brakel, W. H. (2006). Measuring health-related stigma: A literature review. Psychology, Health \& Medicine, 11(3), 307-334. 
Vanable, P. A., Carey, M. P., Blair, D. C., \& Littlewood, R. A. (2006). Impact of HIV-related stigma on health behaviors and psychological adjustment among HIV-positive men and women. AIDS and Behavior, 10(5), 473-482.

Varas-Diaz, N., Serrano-Garcia, I., \& Toro-Alfonso, J. (2005). AIDS-related stigma and social interaction: Puerto Ricans living with HIVIAIDS. Qualitative Health Research, 15(2), 169-187.

Veit, C. T., \& Ware, J. E. (1983). The structure of psychological distress and well-being in general populations. Journal of Consulting and Clinical Psychology, 51(5), 730742.

Vermeer, W., Bos, A. E., Mbwambo, J., Kaaya, S., \& Schaalma, H. P. (2009). Social and cognitive variables predicting voluntary HIV counseling and testing among Tanzanian medical students. Patient Education and Counseling, 75(1), 135-140.

Visser, M. J., Makin, J. D., \& Lehobye, K. (2006). Stigmatizing attitudes of the community towards people living with HIVIAIDS. Journal of Community \& Applied Social Psychology, 16(1), 42-58.

Visser, M. J., Neufeld, S., de Villiers, A., Forsyth, B. W. C., \& Makin, J. D. (2008). To tell or not to tell: South African women's disclosure of HIV status during pregnancy. AIDS Care, 20(9), 1138-1145.

Weiner, B. (1993). AIDS from an attributional perspective. In J. B. Pryor \& G. D. Reeder (Eds.), The social psychology of HIV infection. (pp. 287-302). Hillsdale, NJ: Lawrence Erlbaum.

Weiss, M. G., Ramakrishna, J., \& Somma, D. (2006). Health-related stigma: Rethinking concepts and interventions. Psychology, Health \& Medicine 11(3), 277-287.

White, R. C., \& Carr, R. L. (2005). Homosexuality and HIVIAIDS stigma in Jamaica. Culture, Health and Sexuality, 7(4), 347-359.

Wu, D. Y., Munoz, M., Espiritu, B., Zeladita, J., Sanchez, E., Callacna, M., et al. (2008). Burden of depression among impoverished HIV-positive women in Peru. JAIDS Journal of Acquired Immune Deficiency Syndromes, 48(4), 500-504.

Yee, N., Bailenson, J. N., Urbanek, M., Chang, F., \& Merget, D. (2007). The unbearable likeness of being digital: The persistence of nonverbal social norms in online virtual environments. CyberPsychology \& Behavior, 10(1), 115-121.

Yiu, J. W., Mak, W. W., Ho, W. S., \& Chui, Y. Y. (2010). Effectiveness of a knowledge-contact program in improving nursing students' attitudes and emotional competence in serving people living with HIVIAIDS. Social Science and Medicine, 71(1), 38-44.

Yoshioka, M. R., \& Schustack, A. (2001). Disclosure of HIV status: Cultural issues of Asian patients. AIDS Patient Care and STDs, 15(2), 77-82. 
Zea, M. C., Reisen, C. A., Poppen, P. J., Bianchi, F. T., \& Echeverry, J. J. (2005). Disclosure of HIV status and psychological well-being among Latino gay and bisexual men. AIDS and Behavior, 9(1), 15-26. 
SUMMARY 
Few modern illnesses have been as extensively stigmatized as HIV. The consequences of HIV-related stigma are substantial and include hampered HIV prevention, testing delays, poor treatment adherence, psychological distress in people living with HIV (PLWH), and disrupted social interactions. This dissertation reports on the social and psychological processes involved in the production and experience of HIV-related stigma.

The first part of this dissertation describes the results of comprehensive qualitative research conducted with African, Dutch Antillean, and Surinamese diaspora in the Netherlands. In Chapter 2, the beliefs that underlie and contribute to HIV-related stigma in these communities are investigated. Interviews with both HIV-positive and HIV-negative community members established that beliefs that HIV is highly contagious, that HIV is a very severe disease, and that PLWH are personally responsible for acquiring their HIV infection contribute to HIV-related stigma, as does the belief that PLWH are HIV-positive because they engage in norm-violating behavior such as promiscuity, commercial sex work, and, for Afro-Caribbean diaspora, also homosexuality. These beliefs were found to be exacerbated and perpetuated by cultural taboos on talking about HIV and sexuality.

Chapter 3 describes the manifestations and consequences of HIV-related stigma in African, Dutch Antillean, and Surinamese communities, and delineates the coping strategies employed by PLWH to mitigate the negative social and psychological consequences of HIV-related stigma. In this study, HIV-related stigma was found to manifest as social distance, physical distance, words, and silence, and to have substantial psychological, social, and health-related consequences. The psychological consequences of HIV-related stigma were emotional pain, sadness, loneliness, anger, frustration, and internalized stigma. The social consequences included decreased social network size, limited social support, and social isolation, and were found to result from not only enacted stigma but also self-imposed social withdrawal. Also, poor treatment adherence was found to be a health-related consequence. In terms of coping strategies, this study established that PLWH employ both problem-focused and emotion-focused coping strategies to mitigate the negative consequences of stigma. Problem-focused coping strategies reported were selective disclosure, disengagement, affiliating with similar others, seeking social support and, to a lesser extent, activism. Emotion-focused strategies included distraction, positive reappraisal, religious coping, external attributions, disidentification, and acceptance.

Chapter 4 investigates how African, Dutch Antillean, and Surinamese PLWH approach disclosure. Previous research has shown that HIV status disclosure is a reasoned process whereby the costs and benefits to oneself and to others are weighed. As such, understanding disclosure requires understanding the reasons for and against disclosure employed by $\mathrm{PLWH}$. In this study, reasons for nondisclosure and disclosure were established. Reasons 
for nondisclosure were fear of stigmatization, having had previous negative experiences with disclosure, having observed the stigmatization of other $\mathrm{PLWH}$, feeling shame, and wanting to protect others - particularly one's children and family - from stigmatizationby-association and/or worrying, and believing that one's HIV status is a private matter. Participants reported disclosing because they were in a close and supportive relationship, disclosure led to emotional release, disclosure could lead to emotional or financial support, they felt a perceived duty to inform, and they had a desire to educate others about sexual risk-taking. The findings suggest that stigma plays an important role in disclosure decisions among these populations.

Together, the three chapters in the first part of this dissertation follow the process of stigmatization in African and Afro-Caribbean diaspora communities from the beliefs underlying stigma to the manifestations and consequences of HIV-related stigma and subsequent coping while also considering how African and Afro-Caribbean PLWH decide whether or not to disclose their HIV status.

The second part of this dissertation explores some aspects of HIV-related stigma in more detail and expands the study population and sample to include all PLWH living in the Netherlands. The findings reported in this section are also, in contrast to the first part of this dissertation, quantitative in nature.

Chapter 5 investigates, using a cross-sectional survey, which specific stigma experiences are most strongly related to psychological distress across a number of social settings, something that has not previously been done. Most studies investigating the psychological impact of HIV-related stigma employ an aggregate measure of stigma and, although this approach provides useful information about the psychological implications of HIV-related stigma in general, it neglects to acknowledge the possibility that some manifestations in specific settings may be psychologically more detrimental than others. As a result, this study examined participants' experiences of 11 manifestations of HIV-related stigma in 6 social settings. Linear regression analyses were conducted to determine which settingspecific manifestations best predict psychological distress. The results show that three manifestations in family settings, namely receiving advice to conceal one's status, being avoided, and being treated with exaggerated kindness, and one manifestation in health care settings, namely awkward social interaction, best predicted psychological distress in $\mathrm{PLWH}$, thus demonstrating that manifestations of HIV-related stigma do vary according to setting.

Chapter 6 returns to the issue of disclosure and, in particular, to the debate on whether a visible or concealable stigma is more detrimental to PLWH's psychological well-being 
and social lives. The study reported in this chapter investigated HIV-related stigma, psychological distress, self esteem, and social support in a sample comprising people who have concealed their HIV status to all but a selected few (limited disclosers), people who can conceal but chose to be open (full disclosers), and people who had visible symptoms that made concealing difficult (visibly stigmatized). The findings indicate that while visibly stigmatized participants and full disclosers both reported significantly more stigma experiences than limited disclosers, only the visibly stigmatized reported more psychological distress, lower self esteem, and less social support than limited disclosers. This suggests that having a visible stigma is more detrimental than having a concealable stigma. Differences in psychological distress and self esteem between the visibly stigmatized and full disclosers were mediated by social support while differences between the visibly stigmatized and limited disclosers were mediated by both social support and stigma. These findings clearly suggest that social support buffers psychological distress in PLWH.

The final chapter of this dissertation, Chapter 7, is a general discussion of all research findings, both the qualitative findings acquired through research with African, Dutch Antillean, and Surinamese diaspora communities and the quantitative findings gathered through cross-sectional research with the general PLWH population in the Netherlands. This chapter not only summarizes the findings of the studies reported in this dissertation and discusses them in the context of the current literature; it also discusses the role of culture in understanding stigma, claiming that HIV-related stigma is a relatively ubiquitous phenomenon. Before concluding, this chapter reflects on the methods used to conduct the research reported in this dissertation, outlines implications of the research findings, and provides recommendations for future research. 
SAMENVATTING 
Mensen met HIV behoren tot één van de meest gestigmatiseerde groepen in onze samenleving. HIV stigma heeft ernstige gevolgen voor sociale interacties, psychologisch welbevinden en de gezondheid. Zo leidt HIV stigma niet alleen tot het verbreken van sociale relaties en een verminderd psychologisch welbevinden, maar belemmert het ook HIV preventie, het tijdig testen op HIV en therapietrouw. Dit proefschrift gaat dieper in op de sociale en psychologische processen, die gerelateerd zijn aan HIV stigma.

Het eerste deel van dit proefschrift beschrijft de resultaten van uitgebreid kwalitatief onderzoek naar HIV stigma onder Afrikaanse, Antilliaanse en Surinaamse gemeenschappen in Nederland.

In hoofdstuk 2 zijn de determinanten van HIV stigma onder Afrikaanse, Surinaamse en Antilliaanse gemeenschappen beschreven. Uit interviews die gehouden zijn onder HIV positieve en HIV negatieve leden van deze gemeenschappen blijkt dat ideeën over de besmettelijkheid, ernst en persoonlijke verantwoordelijkheid voor het ontstaan van de ziekte gerelateerd zijn aan HIV stigma. Daarnaast blijkt ook dat HIV geassocieerd wordt met normovertredend gedrag zoals promiscuïteit, prostitutie en voor Afro-Caribische immigranten, homoseksualiteit. Het culturele taboe om te praten over HIV en seksualiteit versterkt de negatieve beeldvorming over HIV.

Hoofdstuk 3 beschrijft de uitingsvormen en gevolgen van HIV stigma onder Afrikaanse, Antilliaanse en Surinaamse gemeenschappen. Daarnaast wordt onderzocht welke copingstrategieën mensen met HIV in deze gemeenschappen gebruiken om met de negatieve sociale en psychologische gevolgen van HIV stigma om te gaan. In dit onderzoek werd gevonden dat HIV stigma zich uit door sociale afstand, fysieke afstand, woorden en stilte, wat ernstige psychologische, sociale en gezondheidsgerelateerde gevolgen heeft. De psychologische gevolgen van HIV stigma zijn emotionele pijn, verdriet, eenzaamheid, boosheid, frustratie en geïnternaliseerde stigma. De sociale gevolgen zijn een verminderd sociaal netwerk, beperking in sociale steun en sociale isolatie. Deze sociale gevolgen zijn niet alleen een gevolg van stigmatisering, maar ook van een zelf opgelegde terugtrekking uit het sociale leven. Verder is therapietrouw een gezondheidsgerelateerde consequentie. De huidige studie bevestigde dat mensen met HIV zowel probleemgerichte als emotiegerichte copingstrategieën hanteren om met de negatieve gevolgen van stigmatisering om te gaan. Voorbeelden van probleemgerichte copingstrategieën zijn selectieve onthulling van de HIV status, het terugtrekken uit sociale relaties, de omgang met anderen in dezelfde situatie, het zoeken van sociale steun en - hoewel minder toegepast - activisme. Emotiegerichte copingstrategieën zijn afleiding zoeken, positieve herwaardering, religieuze coping, externe attributies, disidentificatie en acceptatie. 
In hoofdstuk 4 staat het onthullen van de HIV status van Afrikaanse, Surinaamse en Antilliaanse mensen met HIV centraal. Eerder onderzoek heeft aangetoond dat HIV status onthulling een beredeneerd proces is, waarbij de kosten en baten worden afgewogen. In deze studie zijn de redenen voor het al dan niet onthullen van de HIV status onderzocht. Redenen voor het niet onthullen zijn angst voor stigmatisering, eerdere negatieve ervaringen bij een onthulling, het opmerken van stigmatisering van andere mensen met HIV, schaamte, de behoefte om anderen - in het bijzonder kinderen en familie - (emotioneel) te beschermen en te voorkomen dat zij ook gestigmatiseerd worden, en de overtuiging dat de HIV status een privé zaak is. Deelnemers aan het onderzoek melden tot onthulling over te gaan als zij een nauwe band met iemand hadden waarin wederzijdse steun ervaren werd of wanneer het zou kunnen leiden tot emotionele bevrijding, of tot emotionele of financiële steun of wanneer men het een plicht vond om anderen te informeren en men anderen graag zou willen voorlichten over seksueel risicogedrag. Deze bevindingen suggereren dat stigmatisering een belangrijke rol speelt bij het besluit om de HIV status te onthullen aan anderen.

De drie hoofdstukken van het eerste deel van het proefschrift beschrijven dus het proces van HIV stigma onder Afrikaanse, Surinaamse en Antilliaanse migranten. Daarbij is zowel gekeken naar de oorzaken van stigmatisering, de uitingsvormen van stigmatisering en de gevolgen van stigmatisering. Bovendien zijn de manieren waarop men met de negatieve gevolgen van HIV stigma omgaat onderzocht. Daarnaast is dieper ingegaan op de redenen voor het al dan niet onthullen van de HIV status .

Het tweede deel van het proefschrift gaat dieper in op een aantal andere aspecten van HIV stigma en verbreedt de onderzoeksgroep naar de gehele populatie van mensen met HIV in Nederland. De bevindingen in dit tweede deel van het proefschrift zijn kwantitatief van aard.

Hoofdstuk 5 beschrijft welke uitingsvormen van HIV stigma in verschillende sociale contexten het meest ongunstig zijn voor het psychologisch welbevinden van mensen met HIV, iets wat nog niet eerder is onderzocht. In dit onderzoek is gekeken naar 11 uitingsvormen van HIV stigma in 6 verschillende sociale contexten. Uit lineaire regressie-analyses komt naar voren de volgende uitingsvormen van stigmatisering door familieleden het psychologisch welbevinden voorspelden: het advies krijgen om de HIV status niet te onthullen, vermeden worden en overdreven aardig doen. Eén uitingsvorm in de gezondheidszorgbleek een significante invloed te hebben op het psychologisch welbevinden, namelijk ongemakkelijke sociale interactie.

Hoofdstuk 6 richt zich wederom op het onthullen van de HIV status en gaat dieper in op de vraag of openheid of geslotenheid over de HIV status gunstiger is voor het 
psychologisch welbevinden. Hierbij wordt ook de rol van zichtbaarheid van de symptomen onderzocht. In het onderzoek is gekeken naar verschillen in HIV stigma, psychologische stress, zelfwaardering en sociale steun voor mensen die hun HIV status voor bijna iedereen verborgen hielden (beperkte onthullers), mensen die open zijn over hun HIV status (volledige onthullers) en mensen die zichtbare symptomen hebben (zichtbaar gestigmatiseerden). Het onderzoek laat zien dat zichtbaar gestigmatiseerden en volledige onthullers meer stigmatisering ervoeren dan beperkte onthullers. In tegenstelling tot de volledige onthullers, de zichtbaar gestigmatiseerden echter meer psychologische stress, hadden een lagere zelfwaardering en minder sociale steun dan de beperkte onthullers. Dit suggereert dat het hebben van een zichtbaar stigma minder gunstig is dan het hebben van een stigma dat verhuld kan worden. Verschillen in psychologische stress en zelfwaardering tussen zichtbaar gestigmatiseerden en volledige onthullers worden gemedieerd door sociale steun, terwijl verschillen tussen zichtbaar gestigmatiseerden en beperkte onthullers worden gemedieerd door zowel sociale steun als stigmatisering. Deze bevindingen impliceren dat sociale steun als buffer fungeert voor psychologische stress bij mensen met HIV.

Het laatste hoofdstuk van dit proefschrift is een algemene discussie over het kwalitatieve onderzoek onder Afrikaanse, Antilliaanse en Surinaamse migrantenpopulaties en het kwantitatieve onderzoek onder de algemene populatie van HIV-geïnfecteerden in Nederland. Dit hoofdstuk vat niet alleen de belangrijkste bevindingen samen, maar relateert deze ook aan eerder onderzoek binnen de stigma literatuur. Daarnaast wordt dieper ingegaan op de vraag of HIV stigma universeel of cultuur-specifiek is. Tot slot wordt de onderzoekmethodologie besproken, worden de praktische implicaties besproken en worden suggesties voor vervolgonderzoek gegeven. 
ACKNOWLEDGEMENTS 
The Positive Living Under Stigma (PLUS) project and this dissertation would not have been possible without the help and support of a number of people. First and foremost, I want to thank Arjan Bos. Arjan, the grand producer of ideas, thank you for coming up with this project and attaining the necessary financing, for providing me with countless opportunities to grow academically and professionally, for always keeping the project's and my interests in mind throughout, and for your willingness to help no matter how complex or trivial the task. I look forward to our ongoing collaboration in the coming years. Thanks also to John Pryor. John, you have challenged me throughout the writing of this dissertation to think beyond the obvious and you have helped me to overcome a longstanding fear, namely SPSS. That's an impressive feat. I also appreciate the phenomenally warm hospitality that you and Hannah extended to me when I came to work with you in Bloomington. I look back on that time with great fondness and hope to be able to do it again sometime down the road. Gerjo Kok, thank you for stepping in when Herman unexpectedly passed away. I appreciate the freedom you have given me to continue working as I had before. Thanks also Harm Hospers, Glenn Reeder, Nanne de Vries, Anton Dijker, and Onno de Zwart for being part of my assessment committee and for devoting valuable time and energy to this process.

Thank you to AIDS Fonds and Abbott (grant numbers 2006092 and 7004) for their financial support and to Iris Shiripinda at STI AIDS Netherlands, Nicole van Kesteren at TNO, Ronald Brands and Maartje Liebregts at the Dutch HIV Association, Mariette Hamers, Evert van Veen, and Onno de Zwart at the Community Health Services in Rotterdam, Stefan Kremer and Juan Walter at the Community Health Services in Amsterdam, and Fetzen de Groot and Renny Poolstra at the Community Health Services in Groningen for their substantial contribution as project committee members for the PLUS project and/or the HIV-related stigma and taboo among ethnic minorities project. Thanks also to Humanitas and the Professional Organization of HIV Consultants for their contribution to data acquisition, and to Marijn de Bruin at Wageningen University and Jeannine Nellen and Jan Prins at the University of Amsterdam's Medical Centre for sharing data acquired previously. Additional thanks to Chantal Rumanzi, Dudly Tijn-a-Kwie, Elizabeth Njeru, Emmy Markelo, Erik Beune, Germain Ackermans, Indira Snip, Iris Shiripinda, Juan Walter, Mikel Haman, Mirte Wibaut, Nadia Mirjals, Pamela Matinde, Rubelto Baker, and Urmy Macnack for conducting interviews, to Anne van Middelaar, Carolien van Wagenberg, Danielle Pool, Joyce van Leendert, Roy van Veldhuizen, Sanne van Lieshout, and Simone Heijnen for transcribing the interviews, and to Lori Mees for checking these for consistency and clarity. Thanks also to Robert Hoogendijk for contributing to data analyses, to all my co-authors for revising manuscript drafts, to Rob Dokter for translating the summary and Dilana Schaafsma for reviewing it, and to Jaring Dijkstra for completing the graphic design for this dissertation. 
I also want thank a few people who have played a fundamental role in my academic development: my parents, my grandparents, Julian Martin, Matt Commers, and Andre Meijer. Thank you for demonstrating faith in my abilities and for showing me what I am capable of. I am beyond grateful. Thanks also to my sister, Larissa, my brother, Ben, my inlaws, Helma, Jan, Tom and Rinie, and to all the friends and colleagues that have supported and encouraged me over the past few years. Through you, I have learned that dissertation-related stress can best be combated with good conversation, good food, and good wine. A special thanks to Fraukje Mevissen. Thanks for your willingness to be my paranymf, for all our mini-counseling and burn out prevention sessions, for quality culinary evenings, for relaxing days at the sauna, and for simply understanding.

Last but not least, Erik, thank you for everything. You are my husband, my best friend, the father of our child to be, my fellow household manager, my handyman, my chef, my chauffeur, my paranymf, and countless other things. I don't think I could ask for anything more. You have changed my life, given me peace, kept me balanced, and there is no one on this planet better equipped to handle a girl working through a dissertation while doing a million other things than you. Thank you for your patience and for your love. 
ACKNOWLEDGEMENTS 
CURRICULUM VITAE 
Sarah Stutterheim was born on October 24th, 1979 in North York, Ontario, Canada. After studying at the University of Alberta in Edmonton, Alberta, Canada, she moved to the Netherlands to study health sciences at Maastricht University. She received her Masters of Science in Health Education and Promotion in 2006 and subsequently taught at the Maastricht University's Department of International Health until 2008. Meanwhile, she initiated the research reported in this dissertation in early 2007 at Maastricht University's Department of Work and Social Psychology while also running a small editing and translation agency geared to academics and other professionals. She completed the research reported in this dissertation in mid 2010 and is currently a post-doctoral researcher at the Open University in the Netherlands where she continues to investigate HIV-related stigma, particularly in the context of the Dutch health care sector. She also maintains her involvement in studies pertaining to vulnerable populations such as ethnic minorities and intravenous drug users. 

